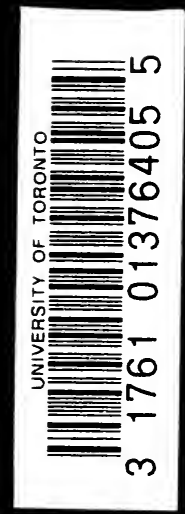




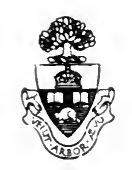

\author{
Presented to the \\ LIBRARY of the \\ UNIVERSITY OF TORONTO \\ by
}

MR. G. POWELL

J. A. CARYETH \& CO. MEDICAL BOOKS 
Aparil 1908
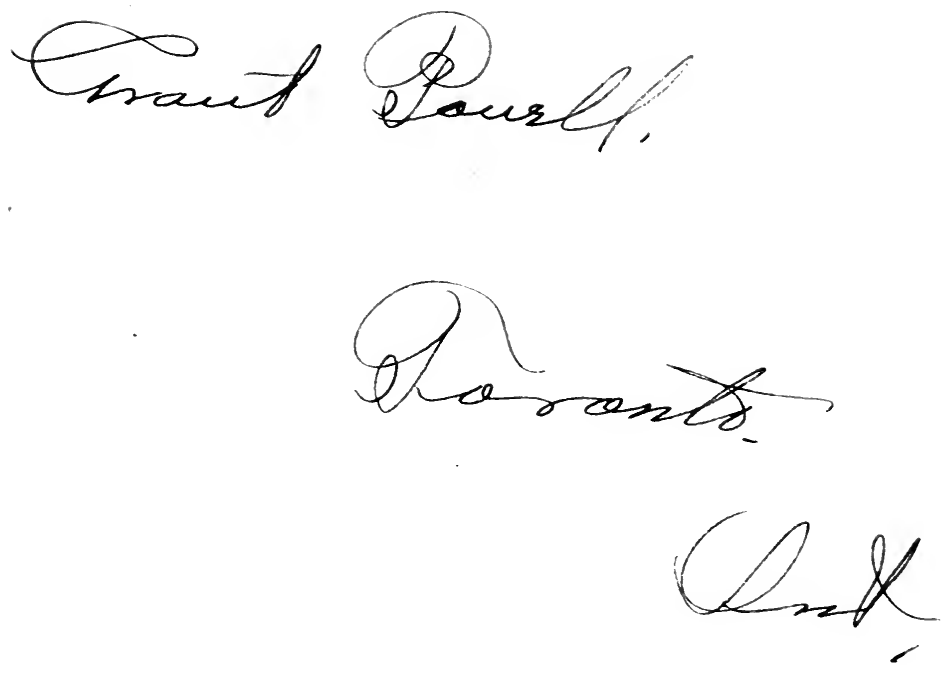



\section{A TEXT-BOOK}

OF

\section{MATERIA MEDICA}

\section{FOR NURSES}

INCLUDING THERAPEUTICS AND TOXICOLOGY

GEORGE P. PAUL, M.D.

ATTENDING PHYSICIAN AND ADJUNCT RADIOGRAPHER TO THE SAMARITAN HOSPITAL AT TROY, NEW YORK ; AUTHOR OF " NURSING

IN THE ACUTE INFECTIOUS FEVERS"

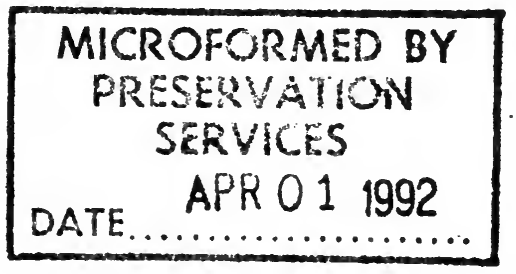

PHILADELPHIA AND LONDON

W. B. SAUNDERS COMPANY 


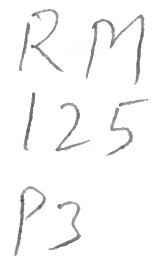

Copyright, 1907,

BY W. B. SAUNDERS COMPANY

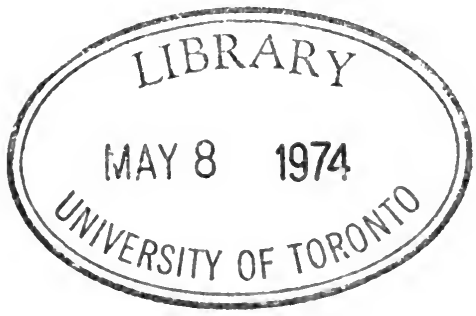


TO THE LATE

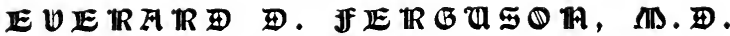

Surgron-in-Chief, Samaritan Hospital, Troy, N. Y. Founder of the New York State Medical Association and, tater, its

President, Secretary, and Treasurer; Sometime

VICE-PRESIDENT OP THE AMBRICAN

Medical Association

IN ADMIRATION OF

HIS GREAT TALENTS AND IN REMEMBRANCE OF

MANY ACTS OF KINDNESS SHOWN TO THE AUTHOR

THIS VOLUME

IS GRATEFULLY INSCRIBED 



\section{PREFACE}

IN the preparation and compilation of a book on this subject for the nurse many difficulties are encountered as to the knowledge to be incorporated in the text and that which should be omitted.

The author has essayed to use only such material as will be of aid to the reader, and has arranged the text in such a manner as he thinks will best meet the demands of the nurse.

The subject matter is arranged in six parts. Part I. consists of General Considerations, which will be found of great importance before beginning the study. of the individual drugs. To Part II. special attention is directed. The drugs of this section are those of a recognized value. They are arranged alphabetically for convenience and, as the author believes, the proper way, as it is impossible to properly classify drugs according to their action, as the majority of drugs have several equally important actions. The physiologic action of the drug is arranged according to the action of the drug and not the organ acted upon, thus, with a glance, the full action of the drug may be seen. Another characteristic of the text is the section on Pretoxic Signs or the warnings of the full action or the beginning toxic effects of the drug, which, if heeded, may prevent many cases of drug poisoning. It is 
necessary that the nurse should known these signs. Under Administration, many useful points as to when and how drugs should be exhibited are given. Part III. includes drugs of minor importance, which, although not used as frequently as those discussed in Part II., are nevertheless worthy of consideration. Part IV. contains the newer preparations much employed by some physicians. Simple mixtures and combinations are not given place, only those of definite chemic union are considered. Part V. relates to practical therapeutic procedures, which will readily be seen to be of prime importance to the nurse. Part VI. includes tables of the percentage strengths of the official drug preparations; of common synonyms; of relative weights and measures.

In conclusion, the author wishes to state that he has been very painstaking in order that he might present this book to the nurse as a concise, accurate, and practical work, and that it might form a valuable and essential part of her equipment.

JANUARY, 1907.

G. P. P. 


\section{CONTENTS}

PAGE

Part I._General Considerations . . . . . . . . . . . I I

Definitions, I I-Drug Constituents, I I-Drug Preparations, 12-Drug Actions, 14-Drug Administration, 21-Drug Classification, 26.

Part II.-General Materia Medica, Therapeutics, and ToxicologY . . . . . . . . . . . . . . . . . . 40

PART III.-Drugs of MiNor IMPORtTANCE . . . . . . . . I65

Part IV.-Newer Medicinal Agents . . . . . . . . . I78

Part V.-Practical Therapeutics . . . . . . . . . . . I85

Hydrotherapy, I85-Hypodermic Medication, I92-Antiseptics and Disinfectants, I94-Disinfection, 197-Topical Medication, 200-Application of Heat, 206-Rectal Alimentation and Medication, 209-Antitoxins and Serums, 212.

PART VI.-ADDENDA . . . . . . . . . . . . . . 215

Strength of Drug Preparations, 215-Synonyms, 220-

Weights and Measures, 224.

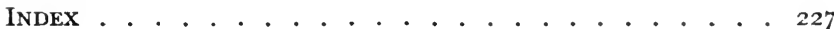




\section{PART I}

\section{General Considerations}

\section{DEFINITIONS}

Materia Medica.-The science that treats of the source, preparation, and properties of agents used as medicines.

Therapeutics.-The science which treats of the application of remedial agents in the treatment of diseased conditions.

Toxicology.-The science which treats of poisons: their nature, detection, effects, and the prevention of their ill effects.

Pharmacopeia.-An authority on the strength, purity, selection, and preparation of medicinal agents.

\section{DRUG CONSTITUENTS}

Alkaloid.-A nitrogenous vegetable base, as a rule representing the active principle of a drug. They are generally insoluble in water, but soluble to varying extents in alcohol, ether, chloroform, and benzin. They form salts with acids which are generally soluble in water. The Latin termination of the name is ina and the English in; e.g., Morphina-Morphin. 
Amaroid.-A bitter vegetable principle.

Glucosid.-A vegetable principle composed of glucose and some other substance. The Latin termination is inum and the English in; e.g., DigitalinumDigitalin.

Stearopten.-A crystallized hydrocarbon deposited from volatile oils; e. g., Camphor and Menthol.

\section{DRUG PREPARATIONS}

The names of the various preparations are arranged as follows, first is the English name, then the Latin name, and then the Latin genitive form, which is the one most used in prescription writing :

Cerate. Ceratum. Cerati.-A preparation for external use whose base is wax, as Ceratum Camphoræ or Camphor Cerate.

Decoction. Decoctum. Decocti.-A liquid preparation of a drug obtained by boiling the drug in water and then straining it, as Decoctum Taraxaci or Decoction of Dandelion.

Elixir. Elixir. Elixir.-A liquid preparation which is really a syrup containing much alcohol, as Elixir Phosphori or Elixir of Phosphorus.

Emulsion. Emulsum. Emulsi.-A liquid preparation in which an oily substance is held suspended in water by gum acacia, yelk of egg, or other agent, as Emulsum Olei Morrhure or Emulsion of Cod-liver Oil.

Extract. Extractum. Extracti-A solid preparation of a drug obtained by evaporating a solution of the drug, as Extractum Opii or Extract of Opium.

Fluidextract. Fluidextractum. Fluidextracti.-A liquid preparation of a drug, one minim of which is 
equivalent to one grain of the drug, as Fluidextractum Aconiti or Fluidextract of Aconite.

Glycerite. Glyceritum. Glyceriti.-A solution of a drug in glycerin, as Glyceritum Amyli or Glycerite of Starch.

Infusion. Infusum. Infusi.-An aqueous solution of a drug obtained by pouring boiling water on the drug and then allowing it to cool and strain, as Infusum Digitalis or Infusion of Digitalis.

Liniment. Linimentum. Linimenti.-A preparation for external use, consisting of strong drugs in an oily base, as Linimentum Belladonnæ or Belladonna Liniment.

Mixture. Mistura. Misturæ.-A preparation in which a drug is held suspended in water by some viscid substance, as Mistura Cretæe or Chalk Mixture.

Mucilage. Mucilago. Mucilaginis.-A watery solution of a gum, as Mucilago Acaciæ or Mucilage of Acacia.

Ointment. Unguentum. Unguenti-A preparation of $a$ drug incorporated with a fatty base and having a semi-solid consistence, as Unguentum Iodi or Iodin Ointment.

Oleate. Oleatum. Oleati.-A solution of a drug in oleic acid, as Oleatum Hydrargyri or Mercury Oleate.

Plaster. Emplastrum. Emplastri.-A preparation in which the drug is incorporated with some adhesive substance as resin, as Emplastrum Belladonnæe or Belladonna Plaster.

Powder. Pulvis. Pulveris.-A finely powdered drug, as Pulvis Opii or Powder of Opium.

Solution. Liquor. Liquoris.-A watery solution 
of non-volatile substances, as Liquor Ferri Chloridi or Solution of Iron Chlorid.

Spirit. Spiritus. Spiritus.-A solution of a volatile substance in alcohol, as Spiritus Ammoniæ or Spirit of Ammonia.

Suppositories. Suppositoria. Suppositorium.-Preparations whose base is cocoa butter, as Suppositoria Glycerini or Glycerin Suppositories.

Syrup. Syrupus. Syrupi-A preparation of a drug in a concentrated solution of sugar, as Syrupus Rhei or Syrup of Rhubarb.

Tincture. Tinctura. Tincturæ.-An alcoholic solution of a non-volatile substance, as Tinctura Nucis Vomicæe or Tincture of Nux Vomica.

Vinegar. Acetum. Aceti.-A solution of a drug in dilute acetic acid, as Acetum Scillæ or Vinegar of Squill.

Water. Aqua. Aquæ.-A solution of a volatile substance in water, as Aqua Chloroformi or Chloroform-water.

Wine. Vinum. Vini.-A solution of a drug in white wine, as Vinum Opii or Wine of Opium.

\section{DRUG ACTIONS}

Alterative.-A drug which modifies metabolism and overcomes morbid processes.

Analgesic.-A drug which when given internally relieves pain. Also known as a general anodyne.

Acetanilid Antipyrin Atropin

Bromids Caffein Camphor Monobromate

Codein Croton Chloral Morphin

Opium Phenacetin Salicylates

Preparations 
Anaphrodisiac.-A drug which depresses the sexual function.

Belladonna Bromids Camphor

Camphor Monobromate Chloral Digitalis

Hyoscin

Hyoscyamus Lupulin

Opium

Valerian

Anesthetic.-A drug producing local or general insensibility.

Local:

Carbolic Acid

Cocain

Ether

Ethyl Chlorid

Eucain

Holocain

Menthol

General :

Chloroform

Ether

Ethyl Bromid

Nitrous Oxid

Anodyne, Local.-A drug which when applied locally will relieve pain.

Aconite

Camphor

Ichthyol

Turpentine
Belladonna

Chloroform

Menthol

Volatile Oils
Carbolic Acid

Guaiacol

Opium

Antacid.-A drug rendering acid solutions alkaline or neutral.

Ammonia-water Chalk Lime-water

Magnesia Potassium Salts Sodium Salts

Anthelmintic. - A drug that expels intestinal worms.

Aspidium

Male Fern

Quassia
Chenopodium

Pomegranate

Santonin

Turpentine
Kousso

Pumpkin Seeds

Spigelia 
Anti-emetic.-A drug which arrests vomiting. Bismuth Salts Bromids Cerium Oxalate Cocain Creosote Menthol Opium Prussic Acid

Antigalactagogue.-A drug which decreases or stops the flow of milk.

Belladonna

Camphor Saline Purgatives

Opium

Antihydrotic.-A drug which diminishes the excretion of sweat.

Agaricin

Camphoric Acid

Atropin Sulphuric Acid Tannic Acid

Antiperiodic. - A drug which prevents the periodic return of symptoms.

Arsenous Acid Picric Acid

Quinin

Antipyretic.-A drug which reduces fever. Acetanilid Aconite

Benzoic Acid

Carbolic Acid

Phenacetin

Quinin

Salicylates
Belladonna

Picrotoxin

Antiseptic.-A drug which prevents the growth and development of bacteria.

Boric Acid

Creosote

Ichthyol

Potassium
Carbolic Acid Formaldehyd Hydrogen Peroxid Lysol Silver Nitrate Thymol

\section{Permanganate}

Antisialagogue.-A drug which decreases the flow of saliva.
Atropin
Belladonna
Myrrh
Opium
Potassium Chlorate 
Antispasmodic.-A drug which tends to overcome muscular spasm.
Amyl Nitrite Atropin
Belladonna

Bromids

Camphor Monobromate Chloral

Hyoscin

Hyoscyamus

Nitroglycerin

Opium

Valerian

Aperient.-A mild cathartic.

Aphrodisiac.-A drug which stimulates the sexual function.

$\begin{array}{lll}\text { Cantharides } & \text { Damiana } & \text { Nux Vomica } \\ \text { Phosphorus } & \text { Saw Palmetto } & \text { Strychnin }\end{array}$

Astringent.-A drug which lessens secretions and discharges by contracting the tissues.

Acetic Acid

Bismuth Salts Copper Salts

Gallic Acid

Hydrastis

Iron Salts

Lead Salts

Silver Nitrate Tannic Acid

Zinc Salts

Cardiac Sedative.-A drug which lessens the heart's power and energy.

Aconite Bromids Gelsemium

Prussic Acid Veratrum Viride

Cardiac Stimulant.-A drug which increases the action and power of the heart.

Adonis Vernalis Ammonia Atropin

Barium Chlorid Belladonna Cactus

Caffein Convallaria Digitalis

Ether Nitroglycerin Spartein

Strophanthus Strychnin

Carminative.-A drug which aids in the expulsion of gas or flatus.

Calamus

Gentian
Calumba

Nux Vomica
Cinchona

Volatile Oils 
Cathartic.-A drug which produces a positive evacuation of the bowels.

Chalybeate.-A drug which has iron as its principal constituent.

Cholagogue.-A drug which stimulates the flow of bile.

Aloes Ammonium Chlorid Benzoic Acid

Calomel Ox-gall

Sodium Choleate Sodium

Podophyllum

Counter-irritant.-A drug which stimulates the peripheral circulation in order to impress or effect distant tissues.
Acetic Acid
Alcohol
Ammonia
Cantharides
. Capsicum
Croton Oil
Iodin
Mustard
Turpentine

Depilatory.-An agent for removing hair.

Diaphoretic.-A drug which increases the excretion of sweat.
Aconite
Dover's Powder Pilocarpin

Digestant.-A drug which has the power of digesting one or more forms of foodstuffs.

$\begin{array}{lll}\text { Amylopsin } & \text { Diastase } & \text { Hydrochloric Acid } \\ \text { Pancreatin } & \text { Papain } & \text { Pepsin }\end{array}$

Disinfectant.-An agent which destroys germ life. Carbolic Acid Chlorin Creolin Formaldehyd Mercury Potassium
Bichlorid
Permanganate

Diuretic.-A drug which increases urinary excretion.

Adonis

Caffein

Diuretin

Scoparius
Apocynum

Convallaria

Juniper

Squill
Arbutin

Digitalis

Nitroglycerin

Spirit of Nitrous Ether 
Ecbolic.-A drug which produces uterine contraction.

Ergot

Quinin

Hydrastis

Savine

Parsley

Emetic.-A drug which provokes vomiting.

$\begin{array}{lll}\text { Alum } & \text { Apomorphin } & \text { Copper Sulphate } \\ \text { Ipecac } & \text { Mustard } & \text { Tartar Emetic } \\ \text { Turpeth Mineral } & \text { Zinc Sulphate } & \end{array}$

Emmenagogue.-A drug which augments the menstrual flow.

Aloin

Apiol

Ergot

Guaiac

Iron Salts

Pennyroyal

Savin

Manganese Compounds

Epispastic.-A drug which when applied to the external surface produces a blister.

Ammonia-water Cantharides Kerosene Oil

Expectorant.-A drug which increases the bronchial secretions.

Ammonium Salts

Antimony

Apomorphin

Ipecac

Licorice

Squill

Terpin Hydrate

Senega

Galactagogue.-A drug which increases the flow of milk.

Extract of Malt Fluidextract of Castor Oil Leaves

Hematinic.-A drug which increases the nemoglobin of the blood.

Arsenic

Cod-liver Oil Iron Compounds

Manganese Salts

Hemostatic.-A drug which arrests hemorrhage.

Adrenalin

Hydrastis

Cotarnin

Ergot

Witch Hazel 
Hypnotic.-A drug which produces sleep which is more or less natural.

$\begin{array}{lll}\text { Bromids } & \text { Chloral } & \text { Chloralamid } \\ \text { Hyoscin } & \text { Opium } & \text { Paraldehyd } \\ \text { Sulfonal } & \text { Trional } & \end{array}$

Laxative.-A mild cathartic.

Cascara Sagrada Glycerin Manna

Senna Sulphur Sweet Oil

Mydriatic.-A drug which causes a dilatation of the pupil.
Atropin
Belladonna
Daturin
Homatropin
Hyoscin
Stramonium

Myotic.-A drug which causes a contraction of the pupil.

Eserin Pilocarpin

Narcotic.-A drug which is capable of producing depression and stupor.

Conium Hyoscin Stramonium

Oxytocic.-Same as Ecbolic.

Ptyalagogue.-A drug which increases the flow of saliva.
Acids
Pilocarpin
Alkalies
Mercury Compounds
Volatile Oils

Purgative.-A drug which produces a free evacuation of the bowels.
Solocynth
-Elaterium
Gamboge
Jalay
Salines
Scammony

Rubefacient.- A drug which causes reddening of the skin when applied locally (See Counter-irritants.)

Sedative, Nerve.-A drug producing quietness of nerve-centers. 
Bromids

Hyoscyamus

Sumbul
Camphor

Lactucarium

Valerian
Cimicifuga

Lupulin

Sialagogue.-Same as Ptyalagogue.

Somnifacient.-Same as Hypnotic.

Soporiflc.-Same as Hypnotic.

Stomachic.-A drug which stimulates the secretory and motor power of the stomach.

Acids

Alkalies

Calumba

Cinchona

Gentian

Hydrastis

Nux Vomica

Quassia

Styptic.-A drug which locally checks hemorrhage. Alum Antipyrin

Copper Sulphate Gallic Acid Hydrogen Peroxid Iron Salts Tannic Acid

Sudoriflc.-Same as Diaphoretic.

Teniacide. - A drug which destroys intestinal worms. (See Anthelmintic.)

Vasoconstrictor.-A drug which causes a narrowing of the blood-vessels.
Cotarnin
Digitalis
Hydrastis
Suprarenal Substance
Ergot

Vasodilator.-A drug producing vascular relaxation.

Amyl Nitrite Nitrites Nitroglycerin

Vesicant.-Same as Epispastic.

\section{DRUG ADMINISTRATION}

When to Administer Drugs.

Bitters and drugs to stimulate the appetite should always be given before meals.

Alkalies given to increase the flow of gastric juice 
should be administered before the meal. If given to neutralize the acidity of the gastric juice the proper time is after the meal.

Acids given to reinforce the natural hydrochloric acid should be administered one-half to one hour after the meal.

Such drugs as mercury, arsenic, iron, iodids, and bromids should not be given when the stomach is empty, but one-half to one hour after meals.

Silver nitrate, when used in the treatment of gastric ulcer, should only be given when the stomach is empty.

Laxatives that are slow in action are to be administered at night, and the more rapid-acting cathartics are best given the first thing in the morning.

Hypnotics are given in the evening before the hour of sleep. When they are given depends on the activity of the drug. Some hypnotics act in half an hour, others not for two or three hours.

Slowly acting drugs, as digitalis, should be administered at long intervals, and rapidly acting drugs, as nitroglycerin and ammonia, at very short intervals.

How to Administer Drugs.

It might be said as a general rule that all liquid preparations should be diluted with water before administering.

Iodids and bromids should never be given in dry form, powder or tablet, but should be dissolved in water and well diluted.

The mincral acids and the tincture of iron chlorid should be taken through a glass tube and well diluted. 
The acids corrode the enamel of the teeth and the iron discolors them.

Saline cathartics if given simply to unload the bowels may be diluted, but when given to aid in the removal of fluid accumulations they should be administered in a concentrated form and very little water given to the patient until the salts have acted well.

In giving bromids to epileptic patients, the use of common salt in the diet should be reduced to a minimum in order to facilitate the maximum action of the bromids.

Arsenic given to patients taking bromids will prevent to a great extent the development of the eruption which so often follows the continuous use of the bromids.

Ergot or the bromids may prevent the occurrence of the ear and head symptoms of cinchonism if they are given in conjunction with the quinin.

Ill-tasting drugs should be given in capsules or konseals.

Methods of Administering Drugs.

By Mouth.-This is the most common of all the methods, and is the best except when very rapid results are desired.

Subcutaneously.-This method is very frequently used when rapid action is wished or when the stomach destroys the virtue of drugs given by mouth. (See Part V.)

By Rectum.-This method is employed when it is impossible to administer drugs by mouth, as in persistent vomiting, semi-consciousness, delirium, certain 
diseases of the stomach, and when drugs by the mouth cause vomiting. Ill-tasting drugs may often be given to advantage by this method. Drugs used for local action on the rectum, bladder, and prostate gland must be applied this way.

By Inunction.-This consists of applying preparations, generally ointments or oily combinations, to the skin and rubbing them in. It is used much as a means of applying mercury (blue mass) in syphilis.

Endermically. - This method is used but little, if at all, at present. It is the application of drugs to the broken skin. A blister is raised and under the elevated epidermis the drug is placed.

Intravascularly.-This is another of the less-used methods. The drug in a soluble form is introduced into a vein. It is employed to a limited extent in administering certain mercury preparations in the treatment of syphilis.

Inhalation.-A much-used method of administering certain remedies in diseases of the respiratory tract.

Fumigation.-This consists of applying easily volatilized drugs by allowing the vapors of the same to come in contact with the skin of the patient. The body of the patient, excepting the head, is enclosed by a tent prepared by wrapping a sheet loosely about the patient. Underneath the tent a lamp and vessel containing the medicament is placed and the vapors allowed to collect under the tent in contact with the patient's body. There is danger of burning the patient, and in order to obviate this the vapor may be conducted to the tent by a tube from the vaporizer, which is placed some distance from the patient. 
Cataphoresis.-This method consists of applying drugs to the skin, as in inunction, and then driving the medicament in by the application of an electric current.

\section{How Much to Administer.}

The size and frequency of a dose of medicine administered depends on many conditions.

Age.-The amount of drug given to elderly people and children differs from that for adults. Children bear certain drugs well and these may be given in very moderate doses, as belladonna; whereas other drugs, as opium, are borne very poorly by the young. To determine the dosage employed in administering drugs to children several methods have been advocated. Young says : "Add 12 to the age of the child and divide the age by this sum. For example: If the child be 3 years old; 3 plus I 2 equals I 5 , and the age 3, divided by 15 , is $\frac{1}{5}$ of the adult dose." Cowling advises: "Divide the next birthday by 24." This would make the dose for a child of 3 years $\frac{4}{2} 4$, or $\frac{1}{6}$ of the adult dose. The dose of a drug for a person over sixty years of age should be less than the adult dose.

Habituation.-The system gradually becomes accustomed to any drug taken for a protracted time, therefore it is often necessary to give a larger dose than usual to affect the system with that drug, as in giving whisky to alcoholics and opium to morphin fiends.

Administration.-Larger doses of the drug are to be given by mouth or rectum than by hypodermic.

Disease.-The character of the disease affects the dosage of the drug. Patients suffering from peritonitis 
can stand larger doses of morphin than otherwise. Digitalis does not affect the heart-rate as readily when the fever is high.

Absorption and Excretion.-Drugs which are absorbed and eliminated rapidly may be given more frequently, as the nitrites, preparations of ammonia, ether, and alcohol. Drugs which accumulate in the system should be given at longer intervals, as digitalis and arsenic.

Condition of the Patient. - Neurotic individuals respond to small doses of drugs, especially if some suggestion has been given. On the other hand, persons with great mental excitement, as maniacs and insane patients, require enormous doses of sedative drugs, as opium and hyoscin, to quiet them.

\section{DRUG CLASSIFICATION}

The classification of drugs is arbitrary. There is no hard and fast rule by which medicinal agents may be classed or grouped. In the following classification the author has followed no prescribed course, but has arranged the drugs as seems to him simple and concise. The drugs are grouped first according to the major anatomic system upon which they act; and then subdivided according to the organ or function which they principally affect.

\section{Drugs Acting on the Respiratory System}

Under this system we will consider only two classes of drugs: Those acting on the bronchial secretions, known as expectorants, and those acting on the respiratory function and called respiratory stimulants.

Expectorants.-An expectorant, as has been pre- 
viously noted, is a drug which increases or modifies the bronchial mucous secretion.

The pathology of bronchitis, laryngitis, etc., teaches us that in the first stage the mucous membrane is greatly reddened by vascular congestion and that the secretion is greatly decreased and tenacious, giving the lining of the larynx and bronchi a dry appearance, and, as the disease progresses, the excretion of mucus is increased.

From the description of the pathology of bronchitis it is easily seen that the treatment of the two stages differs. The drugs used as expectorants are divided into two classes: the sedative and the stimulating. The former are used in the early stage of bronchitis and the latter in the last stage.

The sedative expectorants are:

Ammonium Chlorid Antimony and

Apomorphin

Potassium Tartrate

Ipecac

Licorice

Senega

The stimulating expectorants are:

Benzoin

Eucalyptus
Cubebs

Terpin Hydrate

Respiratory Stimulants.-As the name indicates, these drugs increase the action of the respiratory process. These drugs, as a rule, are stimulants in general, and do not act specifically on the respiratory apparatus. As is commonly known, the respiratory system is seldom depressed without corresponding depression of other vital functions, as the circulatory. It will be appreciated that drugs which stimulate the one function, as a rule, stimulate the other.
Alcohol
Ammonia
Ammonium Carbonate
Atropin
Ether
Strychnin 


\section{Drugs Acting on the Circulatory System}

The drugs of this class will be considered in four divisions: Heart stimulants, heart sedatives, vasoconstrictors, and vasodilators.

Heart Stimulants. - These drugs increase the power of the heart by augmenting the rate or increasing the force of the heart. If, for instance, the heart does a certain amount of work by contracting 72 times per minute, it will do more work by contracting 90 times per minute, provided the contraction was equal in force or even five-sixths as forcible; or if the contractions number only 60 per minute and the force is increased sufficiently, the power of the heart will be greater.

Some drugs, as digitalis and strophanthus, increase the power of the heart by increasing its force but lessening its rate. Other drugs, as ammonia, alcohol, ether, and strychnin, increase both the rate and force of the heart.

The principal heart stimulants are:

$\begin{array}{lll}\text { Adonis Vernalis } & \text { Alcohol } & \text { Ammonia } \\ \text { Barium Chlorid } & \text { Belladonna } & \text { Cactus } \\ \text { Caffein } & \text { Camphor } & \text { Convallaria } \\ \text { Digitalis } & \text { Ether } & \text { Nux Vomica } \\ \text { Spartein } & \text { Squill } & \text { Strophanthus }\end{array}$

Heart Sedatives.- These drugs decrease the power, force, and rate of the heart, and in excessive doses are heart depressants, increasing the rate but decreasing the power of the heart. The principal heart sedatives are: :

Aconite

Bromids

Tartar Emetic

Veratrum Viride 
Vasoconstrictors.-These drugs cause the bloodvessels to become smaller in caliber. By contracting the vessels they place a greater strain on the heart and raise the blood-pressure in the vessels. They act by affecting the centers in the brain or by influencing the nerve filaments in the vessel walls.

Acetanilid Belladonna Cotarnin

Digitalis Ergot Suprarenal Extract

Vasodilators.-These drugs produce a relaxation of the vessel walls and lower the blood-pressure, relieving the heart of considerable work.
Amyl Nitrite
Nitrites
Nitroglycerin

\section{Drugs Acting on the Alimentary System}

The alimentary system is very extensive and is composed of many organs, hence it is natural to suppose that drugs acting on this system should be divided into many groups.

The classes to be considered are: Stomachics, digestives, emetics, anti-emetics, cathartics, antiseptics, astringents, and anthelmintics.

Stomachics. - These drugs increase the motor power of the stomach, stimulate the flow of the juices, sharpen the appetite, and bring about a general tonic effect. The drugs, as a rule, are bitters or of the volatile-oil type. Those of the latter class act as carminatives and assist in expelling gas or flatus from the stomach.
Absinth
Alcohol
Calamus
Calumba
Capsicum
Cinchona
Gentian
Ginger
Hydrastis
Nux Vomica
Quassia
Rhubarb 
Digestives.-These drugs are divided into two classes, according to their origin, animal and vegetable. The animal are:

Ingluvin Pancreatin Pepsin

The vegetable are:

Bromelin Diastase Papain

Emetics.-These drugs produce vomiting. Their uses are several: To empty the stomach when it is overloaded or contains irritating or poisonous material ; to aid in expelling mucus or other substances from the upper respiratory tract; to relax muscular spasm of the respiratory tract, as in asthma and croup. Emetics act either locally on the stomach or centrally in the brain.

Apomorphin

Tartar Emetic
Ipecac

Zinc Sulphate

Anti-emetics.-These drugs oppose the emetics and act as gastric sedatives.

Cerium Oxalate Bismuth Preparations Magnesia Prussic Acid

Cathartics.-In this class are many drugs, and they should be subdivided into several groups, depending on their action.

Cathartics are used to cure constipation, to unload the bowels, to remove water from the system, and to affect distant tissues.

As a cure for constipation, those drugs whose action is followed by constipation should not be employed, for the result we wish to attain is thus defeated. Such drugs as cascara and sodium phosphate, which have a tonic action on the bowels, are of service.

In diseases accompanied by the collection of fluids 
in the tissues, cathartics which produce copious watery movements are of great value. These cathartics are known as the hydragogue purgatives, and are represented by salines, jalap, and mercury.

In diseases of the acute inflammatory type, it is often useful to administer drugs which irritate the intestinal tract, thus drawing blood from the affected parts. Such drugs as croton oil and scammony are typical.

Cathartics may be classed as follows :

Simple laxatives, or those drugs which produce a slight movement of the bowels.

Cassia Fistula Cascara Sagrada Glycerin

Licorice Manna Prune

Senna

Purgatives, or those which produce more copious bowel movements.
Aloes
Calomel
Castor Oil
Podophyllum
Rhubarb

Drastic purgatives are very vigorous-acting drugs. They produce marked irritation and excessive bowel movements.

Colocynth Croton Oil Elaterium

Gamboge Jalap Scammony

Saline cathartics are:

Magnesium Sulphate Potassium Bitartrate (Cream of (Epsom salt)

Tartar)

Sodium Phosphate Sodium Sulphate (Glauber salt) Sodium and Potassium Tartrate (Rochelle salt)

Cholagogue purgatives increase the secretion of bile.
Calomel
Ox-gall
Podophyllum

Intestinal Antiseptics.-These drugs tend to over- 
come fermentation and bacterial processes in the intestinal canal.

$\begin{array}{lll}\text { Creosote } & \text { Guaiacol } & \text { Naphtol } \\ \text { Ox-gall } & \text { Sulphocarbolates } & \text { Thymol }\end{array}$

Intestinal Astringents.-These drugs check the flow of intestinal juices, decrease the motor power to some extent, and are useful in overcoming diarrheal conditions.
Alum
Chalk
Catechu
Gallic Acid
Kino
Sulphuric Acid

Tannic Acid

Zinc Sulphate

Anthelmintics. - These agents are divided into three classes, depending on the parasite on which they especially act.

On the Tapeworm:

Aspidium Cusso Pomegranate

Pumpkin Seed Thymol

On the Round-worm:

Aloes

Santonin

Spigelia

Turpentine

On the Pin-or Seat-worm:

Quassia

Salicylic Acid Salt

Drugs Acting on the Nervous System

This class includes drugs acting on the nervous tissues, whether brain, cord, or peripheral nerves.

Anesthetics.-These drugs produce insensibility, either by their action on the brain centers or locally. These agents are principally used by the surgeon, but are also employed by the physician in overcoming spasms, convulsions, epileptic and eclamptic seizures, and for the relief of intense pain. 
The principal general anesthetics are:
Chloroform
Ether
Nitrous Oxid

The local anesthetics are:

Cocain Ethyl Chlorid Eucain

Hypnotics.-

Bromids

Chloral Hydrate Chloralamid

Hyoscin

Opium

Sulfonal

Trional

Nerve Sedatives.-Under this heading are included drugs which are commonly known as antispasmodics, and such as are generally called motor depressants.
Asafetida
Bromids
Bromoform
Camphor
Camphor
Cannabis Indica
Monobromate
Gelsemium
Hops
Hyoscyamus
Musk
Physostigma
Stramonium

\section{Valerian}

Analgesics.-These drugs are given internally for the relief of pain. It is well to note that nearly all members of this class are more or less depressing to the cardiac function and should be used with caution, especially those of the coal-tar series, and should be reinforced by stimulants.
Acetanilid
Aconite
Ammonium
Chlorid
Antipyrin
Belladonna
Camphor
Monobromate
Cannabis Indica
Cimicifuga
Colchicum
Gelsemium
Oil of Gaultheria Opium
Phenacetin
Salicylates 
Drugs Acting on the Nervous Mechanism of the Eye.-

Mydriatics:

Belladonna

Cocain

Myotics:

Physostigmin or Eserin

Homatropin

Pilocarpin

\section{Drugs Acting on the Urinary Tract}

The drugs of this class are divided into three groups : The diuretics, or those increasing the flow of urine; the antiseptics, or those which render the urine more or less antiseptic; the stimulants, which form an indeterminate group and comprise those drugs which act especially on the mucous membrane of the bladder and lower urinary tract, which is stimulated slightly by these drugs during their excretion.

Diuretics.-These are best studied in three groups, depending on their mode of action. Some of the drugs act directly on the kidney tissue and are known as the stimulating diuretics:

Juniper Cantharides Turpentine Uva Ursi

The second class of diuretics produces an increase of urine by altering the blood supply of the kidney. They may be cardiac stimulants, thus increasing the amount of blood to the kidney; they may act on the general vascular system, increasing the tension and thereby increase the blood in the kidneys; or they may simply dilate the renal vessels. These drugs, for want of better nomenclature, will be designated as systemic diuretics:
Adonis Vernalis
Caffein
Calomel Convallaria
Digitalis Scoparius
Squill
Strophanthus 
The third group consists of drugs derived principally from the saline division. These render the urine bland, and thus tend to increase the flow of this excretion. Lithium Lithium Citrate Potassium Acetate Carbonate

Potassium

Citrate

Potassium

Sodium Acetate

Nitrate

Sodium Citrate Strontium Salts

Diuretics are used for several purposes : To increase the urinary flow in diseases in which it is partially suppressed, to remove abnormal collections of fluid, as in effusive pleurisy, pericarditis or peritonitis, etc., and to increase the excretion of poisons and toxins in uremia, eclampsia, and toxemia.

Urinary Antiseptics.-The drugs of this class are excreted in the urine and thus tend to render this fluid feebly antiseptic, either by their own virtue or by products formed from these drugs while in the system.

\section{Benzoates Benzoic Acid Boric Acid Methylene-blue Sandalwood Urotropin}

Urinary Stimulants.-This is an arbitrary class, but comprises those drugs which, when excreted in the urine, lessen its irritation, act as stimulants to the mucous membrane of the bladder and urethra, and may act as sedatives.

These drugs are not used for the purpose of stimulating the flow of urine, but for their local action on the lower urinary tract.

$\begin{array}{lll}\text { Boric Acid } & \text { Buchu } & \text { Copaiba } \\ \text { Cubebs } & \text { Hyoscyamus } & \text { Sandalwood } \\ \text { Triticum } & \text { Turpentine } & \text { Uva Ursi } \\ & \text { Zea Mays } & \end{array}$




\section{Drugs Acting on the Genital Tract}

The drugs of this class will be considered in two groups: Those acting on the menstrual function and those affecting the uterine musculature.

Emmenagogues cause the menstrual flow to become normal in amount, when for any reason it is pathologically absent or decreased in quantity. These drugs are of uncertain action. They act on the genital organs primarily or produce results by influencing the general system.

The former type is represented by:

Apiol

Rue
Manganese

Savin
Pennyroyal

Tansy

The latter type by:
Aloes
Cathartics
Cod-liver Oil
Iron Preparations Strychnin

Uterine Stimulants.-Included in this group are those drugs acting on the uterine muscles, causing their contraction. They are used after labor to aid the uterus in becoming firm and small.
Cotton-root Bark
Ergot
Hydrastis

\section{Drugs Affecting Metabolism}

This class is more or less ill defined and includes those drugs which aid in building up tissues and fluids of the body or help the system to overcome morbid processes. These drugs are called altcratives.

Arsenic Compounds

Colchicum

Iodids

Manganese Compounds

Phosphorus Compounds
Cod-liver Oil

Ichthyol

Iron Preparations

Mercurials

Thyroid Extract 
Drugs Acting on the Heat Mechanism

The drugs considered in this section are those which reduce the temperature, and are called antipyretics. Their action is accomplished by increasing the dissipation of heat or decreasing heat production.

$\begin{array}{lll}\text { Acetanilid } & \text { Aconite } & \text { Alcohol } \\ \text { Antipyrin } & \text { Benzoates } & \text { Cinchona } \\ \text { Guaiacol } & \text { Phenacetin } & \text { Picric Acid } \\ \text { Resorcin } & \text { Salicylates } & \end{array}$

\section{Drugs Acting on the Excretory Apparatus}

Among the principal channels of secretion and excretion are the bowels, kidneys, skin, salivary glands, and breasts. Drugs acting on the bowels and kidneys have been considered.

\section{Drugs Affecting the Sweat Glands}

Antihidrotics are drugs which decrease the excretion of sweat.

Agaricin Belladonna Camphoric Acid Formaldehyd Gallic Acid Tannic Acid

Diaphoretics, or drugs increasing perspiration.

Aconite

Pilocarpin
Camphor

Spirit of Nitrous Ether

Drugs Affecting the Salivary Glands

Sialagogues.-

Acids

Alkalies

Iodids

Mercurials
Antisialagogues.-

Belladonna

Borax

Myrrh

Opium 


\section{Drugs Affecting the Mammary Glands}

At times it is necessary either to increase or diminish the mammary secretion.

Galactagogues.-

Ammonium Chlorid

Castor Oil (Fluidextract)

Malt Extract

Pilocarpin
Antigalactagogues.Belladonna

Camphor

Ergot

Saline Purgatives

\section{Drugs Acting Externally}

The members of this division will be considered in four classes : Counter-irritants, antiseptics, vulneraries, and astringents.

Counter-irritants are those agents which when applied locally will stimulate the peripheral circulation, produce a localized increase of blood or serum in the part, and thus relieve more distant tissues. They relieve pain by reducing the tension and congestion of the affected area; they aid in the absorption of fluid accumulations; they are antiphlogistic, $i$. e., relieve inflammation.

The counter-irritants are classified according to the degree of action exerted. If only reddening of the skin is produced the drug is called a rubefacient. If blisters or vesicals are formed, a vesicant or epispastic. If tissues are destroyed, a caustic or escharotic.

Rubefacients :
Ammonia
Capsicum
Chloroform
Ginger
Iodin
Mustard
Turpentine

Vesicants :

Ammonia

Cantharides

Croton Oil

Kerosene Oil 


\section{Caustics:}

Acetic Acid Arsenous Acid Chromic Acid

Nitric Acid Potassium Bichromate

Silver Nitrate Sulphuric Acid Zinc Chlorid

Antiseptics.-These agents are used principally in surgery to retard or check bacterial growth and life. The most-employed strength is noted after the name of the drug.

Antipyrin

Carbolic Acid

$$
(\mathrm{I}-2 \mathrm{O})
$$

HydrogenPeroxid Iodoform

\section{(3 per cent.)}

Potassium Permanganate

$$
\text { (I-IOOO) }
$$

Benzoic Acid Boric Acid

$$
\text { ( I-25) }
$$

Formaldehyd

$$
\text { (I-IOO) }
$$

Mercury Bichlorid

$$
\text { ( } \mathrm{I}-\mathrm{IOOO})
$$

Resorcin

Vulneraries are those drugs which aid in the healing of wounds by stimulating the formation of granulation tissue and encouraging cicatrization.

Balsam of Peru Chrysarobin Ichthyol

\section{Silver Nitrate}

Astringents when applied locally contract the tissues and diminish any discharge from wounds or the cutaneous surface.
Alum
Hydrastis
Iron Salts
Lead Salts
Mineral Acids Tannic Acid
Zinc Salts




\section{PART II}

\section{General Materia Medica, Therapeutics, AND TOXICOLOGY}

(Note.-Drugs marked $(*)$ are found in Part III.)

\section{ABSINTH $\left(^{*}\right)$}

\section{ACACIA $(*)$}

\section{ACETANIIID}

Derivation.-A coal-tar product produced by the action of glacial acetic acid on anilin.

Synonyms.-Antifebrin, Phenylacetamid.

Solubility.-Acetanilid is soluble in water $(200$ parts), alcohol (5 parts), ether (I 8 parts), chloroform (freely).

Properties.-It is a white, crystalline, odorless powder with a burning taste:

Incompatibilities.-Sweet spirit of niter, chloral hydrate, bromids, and iodids.

Dosage.-2-Io grains.

Physiologic Action.

Antipyretic.-Acetanilid is a powerful antipyretic. On the normal temperature its action is slight, but 
when given in febrile conditions it rapidly lowers the temperature, which may become subnormal.

Analgesic.-Acetanilid is principally used to relieve pain. The drug acts as a powerful sedative to the sensory nerve tissues, bringing about an anesthetic and analgesic action.

Antiseptic.-This drug also possesses antiseptic properties to some degree, and is much used for this purpose in the treatment of wounds.

Cardiac Depressant.-Acetanilid depresses the heart action very perceptibly in large doses, and on this account should be used with caution in susceptible persons.

Hemostatic. - When taken internally or applied locally, this drug causes contraction of the blood-vessels.

Comparative Action.-Compared to antipyrin, acetanilid produces a stronger pulse, has a more marked action on the blood, and cyanosis is frequent, but it is less powerful as an analgesic and in its local action on the blood-vessels.

Compared to phenacetin, acetanilid is less safe, but more analgesic.

Therapeutic Indications.

As an antipyretic in fevers, influenza, and tuberculosis.

As an analgesic in headaches, migraine, neuralgias, sciatica, rheumatism, influenza, dysmenorrhea, and locomotor ataxia.

As an antiseptic in dressing wounds, ulcers, chancroids, and chancres.

As a hemostatic it is used locally in nosebleed and oozing of blood from wounds. 
Administration.-Acetanilid is best given in the form of tablets or in capsules. Owing to its depressing action on the heart, acetanilid should be reinforced by strychnin, caffein, or camphor. It should be given in small doses to susceptible persons. The author has known 2 grains of this drug to cause cyanosis and cold blue finger-tips in an adult.

Pretoxic Signs.-Slight cyanosis of the lips and face, sweating, and slowness of the pulse.

Toxicology.-After large doses signs of poisoning may rapidly appear, as cyanosis, slow respirations, feeble and irregular pulse, vomiting, cold, moist skin, and collapse.

Management.-Empty the stomach by means of a stomach-pump or tube or with emetics, as tepid water, salt-water, or mustard-water. Stimulate with aromatic spirit of ammonia, strychnin, atropin, or brandy. Apply heat externally by means of hot-water bags, hot bricks, or hot flat-irons.

\section{ACETIC ACID}

Derivation.-A clear, colorless liquid with an acid taste and a vinegar-like odor, obtained by the distillation of wood.

Incompatibilities.-Alkalies and bicarbonates.

Preparations.

Glacial Acetic Acid (99 per cent. strength).

Acetic Acid (36 per cent. strength).-Dose, Io30 minims.

Dilute Acetic Acid (6 per cent. strength).-Dose, I-3 drams.

Vincgar (Acetum) is a form of dilute acctic acid. 
Physiologic Action.

Caustic.-Glacial acetic acid when applied locally reddens the skin and causes formation of blisters. If the action continues it will destroy the tissues.

Astringent.-Given internally or applied locally acetic acid constricts the blood-vessels, astringes the tissues, and thus causes a decrease in the secretion of intestinal juices and sweat.

Stimulant.-When inhaled the drug reflexly stimulates cardiac and respiratory action.

Therapeutic Indications.

As a caustic for removing warts, corns, vegetations, and small growths.

As an astringent in night-sweats of tuberculosis, nosebleed, postpartum hemorrhage, and in diarrhea.

As a stimulant, by inhalation, in asphyxia and syncope.

The inhalation of the vapor of acetic acid or vinegar will at times stop vomiting which has resisted all other treatment.

Toxicology.-Acetic acid in poisonous doses produces vomiting, griping, collapse, convulsions, and paralysis.

Management.-Dilute the poison by giving water. Neutralize it with soap-water, chalk, milk, oils, or white of egg. Stimulate if necessary.

\section{ACONITE}

Derivation.-The root of Aconitum Napellus.

Synonyms.-Monkshood, Wolfsbane.

Constituent. - An alkaloid (Aconitin), which occurs in crystalline and amorphous forms. 
Dosage.- $\frac{1}{2}-2$ grains.

Preparations.

Extract.-Dose, $\frac{1}{8}-\frac{1}{2}$ grain.

Fluidextract.-Dose, $\frac{1}{4}-\mathrm{I}$ minim.

Tincture.-Dose, I-3 minims.

Aconitin (crystalline).-Dose, $\frac{1}{600} \frac{1}{200}$ grain.

Aconitin (amorphous).-Dose, $\frac{1}{40}-\frac{1}{15}$ grain.

Physiologic Action.

Analgesic.-Aconite depresses the ends of the sensory nerve-fibers, causing a tingling sensation and numbness of the parts.

Cardiac Sedative.-In medicinal doses this drug acts as a sedative to the heart, producing a slower and less forcible cardiac action.

Vasodilator.-Aconite lowers the blood-pressure by dilating the blood-vessels and by its action on the heart and vasomotor centers in the brain.

Respiratory Sedative.-The respirations become slower under the action of aconite.

Antipyretic.-From its action on the heart and blood-vessels this drug reduces the temperature in febrile conditions.

Diuretic.-Aconite, being largely eliminated by the kidneys, stimulates these organs and causes an increase in the amount of urine.

Therapeutic Indications.

As an analgesic it is used locally in neuralgias as a liniment. Aconite is given internally for the same action in rheumatism, neuralgias, sciatica, dysmenorrhea, and migraine. As a gastric analgesic it is useful in the vomiting of pregnancy.

As a cardiac and zasomotor sedative it is used in 
hypertrophy of the heart, and cardiac palpitation. In the beginning of acute fevers, as pneumonia, scarlet fever, and erysipelas. In pleurisy, pericarditis, bronchitis, and epistaxis.

Administration.-Aconite is very rapidly absorbed and eliminated, therefore it may be given, frequently repeated, in small doses.

Pretoxic Signs.-Slowness of the pulse, tingling of the skin, and numbness.

Toxicology.-In large doses aconite produces muscular weakness, a slow, weak pulse, which becomes rapid and irregular, collapse, and convulsions.

Management.-Evacuate the stomach by means of a stomach-tube, but do not use emetics, as these cause additional heart depression. Give tannic acid as an antidote. Stimulate with ether, alcohol, strychnin, digitalis, or atropin.

\section{ADONIS VERNALIS $(*)$}

\section{AGARICIN $(*)$}

\section{ALCOHOL}

Derivation.-A liquid obtained by the fermentation and distillation of saccharine substances, and contains 9I per cent. of ethyl hydrate.

Synonyms.-Ethyl Alcohol, Spirit of Wine, Common Alcohol.

Incompatibilities.-Acacia, albumin, and permanganates.

Properties.-It is a colorless, transparent, volatile liquid, with a pleasant odor and a burning taste. 


\section{Preparations.}

Absolute Alcohol contains I per cent. of water. Deodorized Alcohol contains 7.5 per cent. of water. Common Alcohol contains 9 per cent. of water. Dilute Alcohol contains 58.5 per cent. of water. Brandy (Spiritus Vini Gallici) contains about 45 per cent. of alcohol, and is obtained by the distillation of grape wine.

Whisky (Spiritus Frumenti) contains about 47 per cent. of alcohol, and is obtained by the distillation of certain grains, as rye, corn, and wheat.

Rum is obtained by the distillation of molasses, and contains about 42 per cent. of alcohol.

Gin (Spiritus Juniperis Comp.) is common grain spirits distilled with juniper berries, and contains about 45 per cent. of alcohol.

Red Wine (Vinum Rubrum) is obtained by the fermentation of the entire grape, and contains from 7 to 12 per cent. of alcohol. Red wines contain tannic acid and are constipating. The principal red wines are Claret, Bordeaux, and Port.

White Wine (Vinum Album) is obtained by the fermentation of the juice of grapes, and contains from 7 to 12 per cent. of alcohol. The common white wines are Sherry, Madeira, and Catawba.

Champagne is a charged or carbonated wine, containing from 5 to I 5 per cent. of alcohol.

Beer is obtained by the slow fermentation of malted barley and hops, and contains from 2 to 5 per cent. of alcohol. 
Ale is prepared by the rapid fermentation of malted barley and hops, and contains from 4 to 7 per cent. of alcohol.

Physiologic Action.

Cerebrospinal Excitant.-Alcohol acts upon the brain and spinal cord, stimulating the centers to a marked degree. Over-doses cause depression.

Cardiac Stimulant.-Alcohol acts as a powerful cardiac stimulant, producing a more rapid and forcible heart action. The pulse becomes full and the arterial pressure is somewhat elevated. Large doses paralyze the heart.

Antipyretic.-Alcohol in moderate doses causes a fall of body temperature.

Gastric Stimulant.-This drug in minute doses increases the flow of gastric juice, but in large doses retards the action of the digestive ferments.

Large doses of alcohol may depress any of the vital functions and act as a poison.

Therapeutic Indications.

As a cardiac stimulant in acute heart depression, as syncope, collapse, and shock. In cardiac failure or weakness of acute fevers, as pneumonia, typhoid fever, and in poisoning due to aconite, veratrum viride, anesthetics, and snake venom.

Alcohol is also indicated in wasting diseases, in persistent vomiting, as an external application in sweating, and to prevent the bed-sores of typhoid fever.

Administration.-As a stimulant in cardiac depression use either whisky or brandy. In persistent vomiting, brandy or champagne on cracked ice are to be used. 
Toxicology.-Acute poisoning is characterized by flushing of the face, rapid pulse, mental excitement, incoherent speech, vomiting, stupor, and coma.

Management. - Empty the stomach at once by means of a stomach-tube or a strong emetic, as apomorphin ( $\frac{1}{10}$ grain, by hypodermic). Stimulate with aromatic spirit of ammonia, strychnin, digitalis, or strong coffee (per rectum). Apply heat externally.

Chronic poisoning presents the following picture: Tremor, mental impairment, insomnia, nausea or vomiting, dyspepsia, redness of the eyes. In advanced cases, delirium tremens occurs with cerebral excitement, inability to sleep, incoherency of speech, tremor and hallucinations, followed by mania and coma.

Management.-Withdraw the cause (alcohol) and supply the necessary stimulation by giving strychnin nitrate. Encourage the appetite with capsicum and bitters. Aid sleep with hypnotics, quiet delirium and mental excitement with bromids and chloral (use caution). Provide abundant and nourishing diet.

\section{ALOES}

Derivation.-The dried juice of the leaves of Aloe Perryi and Aloe Socotrina.

Constituent.-A crystalline substance called Aloin. Preparations.

Purified Aloes.-Dose, I-1o grains.

Extract.-Dose, $\mathrm{I}-5$ grains.

Tincture.-Dose, $15-60$ minims.

Tincture of Aloes and Myrrh.-Dose, I-2 drams. Aloin.-Dose, $\frac{1}{4-2}$ grains.

Also several pills. 
Physiologic Action.

Cathartic.-Aloes stimulates the flow of bile and intestinal juices. This drug acts especially on the lower end of the bowels. Its action is slow and usually takes from twelve to eighteen hours.

Emmenagogue.-Aloes acts as a stimulating emmenagogue, causing an increased menstrual flow when it is scanty.

Vermifuge.-The drug is a useful anthelmintic for the round-worm and seat-worms.

Therapeutic Indications.-As a cathartic in chronic constipation. It is too slow in action for acute constipation.

As an emmenagogue in atonic amenorrhea accompanied by constipation and anemia.

As an anthelmintic for round- and pin-worms.

Administration.-Aloes or aloin is very seldom given alone, but usually combined with belladonna or strychnin. It should not be employed in constipation accompanied by inflamed piles. As an emmenagogue it is best given with an iron preparation. For seatworms it is used in the form of an enema.

\section{ALUM}

Derivation.-A double salt formed by the action of heat on alum stone and then purified.

Synonyms.-Potassio-aluminum Sulphate, Potash Alum.

Properties.-Alum occurs in large, colorless crystals, and is soluble in water (Io parts), glycerin (freely), but insoluble in alcohol. Heated to redness it becomes Burnt Alum (Alumen Exsiccatum). 
Incompatibilities.-Alkalies, lime-water, lead acetate, tannic acid, and iron salts.

Dosage. - 5-30 grains.

Physiologic Action.

Astringent.-Alum applied externally or given internally coagulates albumin and constringes the tissues, thus hardening the skin and decreasing the cutaneous and alimentary secretions.

Emetic.-This drug, by its irritation on the lining of the stomach, provokes vomiting.

Therapeutic Indications.

As an astringent in epistaxis, sore throat, tonsillitis, leukorrhea, hemorrhages, night-sweats, diarrhea, etc.

As an emetic in acute poisonings and membranous croup.

Administration.-As an emetic alum should be given in doses of $\mathrm{I}$ to 2 drams.

Toxicology.-Very large doses produce vomiting and purging.

\section{AMMONIAC (*) \\ AMMONIUM COMPOUNDS}

\section{AMMONIA-WATER}

Derivation.-A watery solution of ammonia gas of Io per cent. strength.

Properties.-A volatile, colorless liquid, with a very irritating, pungent odor.

Incompatibilities.-Acids, alkaloids, metallic salts, iodin, and chlorin-water.

Dosage.-10-30 minims.

Preparations.

Liniment contains 35 per cent. of ammonia-water. 
Spirit.-Dose, 10-60 minims.

Aromatic Spirit contains ammonia-water, ammonium carbonate, and essential oils. Dose, $\frac{1}{2}-2$ drams.

Stronger Ammonia-water, containing 28 per cent. of ammonia gas. Dose, 4-8 minims, well diluted.

Physiologic Action.

Rubefacient.-Ammonia-water when applied to the skin acts as an irritant and causes redness of the surface.

Cardiac and Respiratory Stimulant.-When inhaled or given internally this drug quickly produces a rapid and forcible heart, and causes an increase of the respiratory rate.

Spinal Stimulant.-Spinal activity is augmented and convulsions may be brought about.

Therapeutic Indications.

As a stimulant in fainting, syncope, sudden cardiac failure of pneumonia, typhoid fever, shock, anesthetization, and in snake-bites.

As a mbefacient it is used in the form of a liniment in neuralgias, rheumatism, and sprains.

Administration.-For rapid stimulation it may be used as an inhalant. Care must be used when this strongly irritating drug is employed by inhalation for patients in an unconscious condition, as burns and serious inflammations of the upper respiratory tract may result. Ammonia preparations should always be well diluted before administering.

Toxicology.-In poisonous doses ammonia produces abdominal colic, vomiting, purging which may become bloody, convulsions, and collapse. 
Management.-Neutralize the poison with acid solutions, as vinegar, lemon-juice, or dilute hydrochloric acid. Give mucilaginous drinks, as albumin-water, olive oil, flaxseed-tea, milk, or chalk-water. Do not use a stomach-tube, as a perforation of the stomach wall may result.

\section{AMMONIUM BENZOATE}

This drug has the same action as Benzoic Acid (to which you are referred).

Dosage.-10-30 grains.

It is soluble in water (6 parts), alcohol (28 parts), glycerin (8 parts).

Incompatibilities.-Iron salts and acids.

\section{AMMONIUM BROMID}

This salt has the same action as Potassium Bromid (to which you are referred), excepting that the ammonium salt is less depressing.

Dosage.-I 5-30 grains.

It is soluble in water (2 parts), alcohol (30 parts).

Incompatibilities.-Mercury and silver salts, acids, and alkaloids.

\section{AMMONIUM CARBONATE}

Synonyms.-Volatile Salt, Sesquicarbonate of Ammonia.

Properties.-A white, transparent substance with an irritating, ammoniac odor. It is soluble in water (5 parts), alcohol (200 parts).

Incompatibilities.-Iron, lead and silver salts, acids, alkaloids, and calomel.

Dosage. - 5-30 grains. 
Preparations.

Aromatic Spirit of Ammonia.-(See under Ammonia-water.)

Solution of Ammonium Acetate.-This is commonly known as the spirit of Mindererus, and is prepared by the action of acetic acid on ammonium carbonate. Dose, I-4 drams.

Physiologic Action.

The action of this salt is the same as that of ammonia-water, and in addition the carbonate is an excellent expectorant and gastric antacid.

Therapeutic Indications.

As a cardiac stimulant and rubefacient it is used the same as ammonia-water. As an expectorant it is useful in acute and chronic bronchitis, pneumonia, and pulmonary tuberculosis. As an antacid in acid dyspepsia.

Administration.- This drug should always be given in solution and well diluted.

\section{AMMONIUM CHLORID}

Synonyms.-Sal Ammoniac, Muriate of Ammonia.

Properties.-It is a white powder with an intensely salty taste. Is soluble in water (3 parts), glycerin ( 5 parts), and nearly insoluble in alcohol.

Dosage. - 5-30 grains.

Preparation.

Lozenge ( $\mathrm{I} \frac{1}{2}$ grains).

Physiologic Action.

Expectorant.-This salt increases the bronchial secretions and makes them thin and watery.

Cholagogue.-Sal ammoniac is said to increase and liquefy the bile. 
Analgesic.-This drug lessens, to a great degree, pain of neuralgic character.

\section{Therapeutic Indications.}

As an expectorant in acute and chronic bronchitis, catarrhal pneumonia, and laryngitis.

As a cholagogue in torpor of the liver, catarrhal jaundice, and hepatic congestion.

As an analgesic in neuralgias, hemicrania, dysmenorrhea, rheumatism, and sciatica.

\section{AMMONIUM IODID}

This salt has the same action as Potassium Iodid (to which you are referred). The ammonium salt is less depressing.

Dosage. - 5-30 grains.

\section{AMMONIUM VALERIANATE}

The action of this drug is about the same as that of Valerian (which see).

Dosage.-2-8 grains.

\section{AMYL NITRITE}

Definition.-A clear, lemon-colored, very volatile liquid, having an odor resembling that of banana.

Dosage.- I-5 minims.

Physiologic Action.

Vasodilator.-Amyl nitrite, when taken by inhalation or by mouth, very rapidly produces great vasodilatation, causing cutaneous flushing, roaring in the ears, fulness of the head, and a rapid, full pulse.

Antispasmodic.-This drug produces great muscular relaxation. 
Comparative Action.-Compared to nitroglycerin, amyl nitrite is much more rapid in action, but the results are of much shorter duration.

Therapeutic Indications.

As a vasodilator in shock, chloroform- and cocainpoisoning, in angina pectoris, syncope, and dyspnea.

As an antispasmodic in angina pectoris, asthma, tetanus, convulsions, epileptic paroxysms, spasmodic dysmenorrhea, hysteric seizures, strychnin-poisoning, and whooping-cough.

Administration.-This drug is most frequently given by inhalation, 3 to 5 drops are placed on a handkerchief and applied to the nostrils. Many drug houses place this preparation on the market in thin glass " pearls," which can be easily broken when needed.

\section{ANTIMONY AND POTASSIUM TARTRATE}

Derivation.-A double salt, formed by the action of antimony oxid on cream of tartar.

Synonyms.-Tartar Emetic, Tartrated Antimony.

Properties.-A white, crystalline powder, without odor, and having a sweetish taste. It is soluble in water (17 parts), glycerin (2 I parts), but insoluble in alcohol.

Incompatibilities.-Tannin, lead salts, and carbonates.

Dosage. $-\frac{1}{30}-\frac{1}{15}$ grain. As an emetic, $\frac{1}{2}$ grain.

Preparations.

Wine.-Dose, 5-60 minims.

Compound Syrup of Squill contains 0.2 per cent. of tartar emetic and 8 per cent. each of fluidextracts of squill and senega. Dose, 5-30 minims. 
Physiologic Action.

Emetic. - Tartar emetic acts upon the stomach walls as an irritant, and causes vomiting with great nausea.

Heart Depressor. - This drug is a powerful cardiac depressant. In small doses it decreases both the force and rate of the heart.

Spinal Depressant.-Antimony depresses the sensory part of the cord, causing cutaneous anesthesia and loss of reflexes. It also produces muscular relaxation by depressing the motor segment of the cord.

Expectorant.-The drug, being partially eliminated by the bronchial mucous membrane, increases the expectoration.

Diaphoretic.-By virtue of its depressing action, tartar emetic increases the excretion of sweat.

Caustic.-Locally, the drug acts as a destroyer of tissue and produces pustules.

Therapeutic Indications.

As an emetic it is but little used on account of its depressing action.

As a heart sedative in the beginning of sthenic diseases, as pneumonia, bronchitis, laryngitis, and acute " colds."

Administration.-This drug should always be used with great caution and its action carefully watched.

Toxicology.-The pulse at first is slow, but soon becomes rapid, weak, and irregular. The skin is cold and moist. Nausea, vomiting, and violent purging are prominent. Collapse soon follows.

Management.-Wash out the stomach and give tannic acid freely. Apply heat externally and stimulate with alcohol, strychnin, and digitalis. 


\section{ANTIPYRIN}

Properties.-Antipyrin is a coal-tar derivative, occurring as a white, crystalline powder, having a bitter taste but no odor. It is soluble in water ( 2 parts), ether ( 52 parts).

Incompatibilities. - Benzoates, calomel, chloral, iodids, saleratus, sweet spirit of niter, and tincture of iron chlorid.

Dosage. - 5-I 5 grains.

Physiologic Action.

Analgesic.-Antipyrin, when given internally or applied locally, depresses the sensory nerve filaments, producing an analgesic effect.

Antipyretic.-This drug reduces the body temperature to a marked degree.

Styptic.-If given internally or applied locally, antipyrin produces a contraction of the smaller bloodvessels.

Antiseptic.-This drug, in small amounts, will stop fermentation. A 4 per cent. solution will destroy the Bacillus communis and prevent spore formation.

Comparative Action.-Antipyrin is more analgesic and has less destructive action on the blood than acetanilid. Antipyrin is more powerful but less safe than phenacetin.

Therapeutic Indications.

As an analgesic in headaches, neuralgias, rheumatism, sciatica, influenza, and dysmenorrhea.

As an antipyretic in influenza and simple febrile disorders.

As an antispasmodic in whooping-cough, chorea, and epilepsy. 
As a styptic in nosebleed, coryza, hay-fever, hemorrhoids, and oozing from wounds.

Administration.-In acute febrile diseases if antipyrin be given after a cold bath the temperature will remain low for a considerable time. The action of antipyrin in susceptible persons should be guarded by a heart stimulant.

Pretoxic Signs.-Lethargy, tendency to sleep, and slow pulse-rate.

Toxicology.-In toxic doses antipyrin produces cyanosis, slow respirations, feeble pulse, vomiting, profuse sweating, and collapse.

Management.-Empty the stomach. Apply heat externally. Give oxygen and stimulate with alcohol, ammonia, digitalis, and strychnin.

\section{APOMORPHIN HYDROCHLORATE}

Derivation.-This salt is formed by heating morphin and hydrochloric acid in a sealed tube.

Properties.-It occurs in small gray crystals, without odor, and having a bitter taste. This salt turns green on exposure to air and light. It is soluble in water (45 parts), alcohol (45 parts).

Dosage. $-\frac{1}{60-\frac{1}{8}}$ grain.

Physiologic Action.

Emetic.-This drug acts as an emetic by stimulating the vomiting center in the medulla.

Expectorant.-Apomorphin to some extent increases the bronchial secretion.

Therapeutic Indications.

As an emetic apomorphin hydrochlorate is useful in cases of acute poisoning, especially in acute alcoholism. 
As an expectorant it is used in acute bronchitis, laryngitis, and other catarrhal disorders of the bronchi and larynx. The author has found this drug of great value as an hypnotic in insomnia of chronic alcoholism.

Administration.-As an emetic, this drug is mostly given as a hypodermic injection in doses of $\frac{1}{20}-\frac{1}{10}$ grain. As an expectorant, in doses varying from $\frac{1}{60}-\frac{1}{20}$ grain. Children do not bear this drug well.

ARNICA $(*)$

\section{ARSENIC COMPOUNDS}

ARSENIC IODID

The action of this drug is about the same as that of arsenous acid.

Dosage. $-\frac{1}{60}-\frac{1}{20}$ grain.

Preparation.

Solution of Arsenic and Mercury Iodids (Liquor Arseni et Hydrargyri Iodidi, or Donovan's Solution).-Dose, I-Io minims.

\section{ARSENIC TRIOXID}

Synonyms.-Arsenous Acid, White Arsenic.

Properties. - It is a white, amorphous or crystalline salt, occurring as a powder or in lumps. It is soluble in water (8o parts), hydrochloric acid (6 parts), and nearly insoluble in alcohol.

Incompatibilities. - Tannic acid, iron salts, and magnesia.

Dosage. $-\frac{1}{60}-\frac{1}{20}$ grain.

Preparations.

Solution of Arsenous Acid.-Dose, I-IO minims. 
Solution of Potassium Arsenite (Liquor Potassii Arsenitis, or Fowler's Solution).-Dose, I-10 minims.

\section{Physiologic Action.}

Caustic.-Arsenic oxid applied externally produces a dissolution of tissue.

Gastro-intestinal Irritant.-This drug produces gastric irritation followed by nausea. In very small doses it acts as a gastric sedative.

Hematinic.-Arsenic has a beneficial action on the blood, increasing the number of red blood cells.

Nerve Sedative. - In small doses arsenic depresses nerve action, and in large doses causes paralysis.

Alterative.-Given internally, arsenic acts on the metabolic processes, causing a check of tissue changes, resulting in an increase in weight of the person. It also causes scaly conditions of the skin to disappear.

Therapeutic Indications.

As a caustic in treating warts, epitheliomata, lupus vulgaris, and corns.

As a gastric sedative in vomiting of pregnancy, and especially for vomiting which occurs after a debauch. In gastrodynia and indigestion of the menopause it is useful.

As a hematinic in anemias, leukemia, and pseudoleukemia.

As a nerve sedative in chorea, asthma, and neuralgia.

As an alterative in diabetes, leukemia, tuberculosis, malarial cachexia, chronic rheumatism, and dry, scaly diseases of the skin.

Administration.-Children bear arsenic preparations well. Always give this drug in well-diluted 
forms. Arsenic applied externally may give rise to toxic symptoms.

Pretoxic Signs.-Morning puffiness below the lower eyelids, slight looseness of the bowels, and vague abdominal pain or colic.

Toxicology.-Acute poisoning is characterized by nausea, vomiting, purging, abdominal pain, cold skin, small, feeble pulse, and collapse.

Management.-Wash out the stomach immediately. Give the antidote, hydrated iron with magnesia, or use magnesia, milk, white of egg. A good antidote is prepared by adding ammonia-water to the tincture of iron chlorid and strain, using the mass which remains in the strainer. Stimulate with alcohol, ammonia, and strychnin. Apply heat externally.

Chronic Poisoning.-The chief signs are anemia, loss of flesh, dyspepsia, cutaneous eruptions, and various paralyses due to neuritis.

Management.-Stop the drug. Aid elimination of the poison from the system by giving the iodids. Tone the muscles by means of electricity.

\section{SODIUM ARSENATE}

Action same as arsenous acid.

Dosage. $\frac{1}{60-\frac{1}{20}}$ grain.

Preparation.

Solution of Sodium Arsenate (Liquor Sodii Arseniatis, or Pearson's Solution).-Dose, I-Io minims.

ASAFETIDA (*)

ASPIDIUM (*) 
BALSAM OF PERU (*)

BARIUM CHLORID (*)

\section{BELLADONNA}

Derivation.-The leaves and roots of Atropa Belladonna.

Synonym.-Deadly Nightshade.

Constituent.-Atropin, an alkaloid.

Dosage.-I-5 grains.

Preparations.

Extract.-Dose, $\frac{1}{8}-\frac{1}{2}$ grain.

Fluidextract.-Dose, $\frac{1}{2}-2$ minims.

Liniment.-Apply locally.

Ointment contains Io per cent. of the extract.

Plaster contains 0.4 per cent. of alkaloid.

Atropin.-Dose, $\frac{1}{120}-\frac{1}{60}$ grain.

Atropin Sulphate.-Same dose as atropin.

Physiologic Action.

Analgesic.-Belladonna, applied locally or given internally, depresses the sensory nerve filaments, producing an anodyne effect.

Antispasmodic.-The motor nerves are slightly affected by this drug, causing a subsidence of muscular spasm.

Secretory Depressor.-Belladonna depresses the ends of the secretory nerves, decreasing the secretion of saliva, sweat, bronchial secretion, and the milk.

Cerebral Excitant.-This drug in moderate doses produces cerebral activity.

Cardiac Stimulant.-The heart's action is increased in rate and force by this drug. 
Respiratory Stimulant.-Belladonna is a powerful respiratory stimulant.

Vasoconstrictor.-This drug primarily contracts the blood-vessels, but in larger doses causes them to dilate, producing a cutaneous flushing.

Mydriatic.-Belladonna, applied locally or given internally, produces dilatation of the pupils.

Therapeutic Indications.

As an analgesic in neuralgias, lumbago, pleurisy, and intercostal neuralgia.

As an antispasmodic in asthma, whooping-cough, laryngismus stridulus, dysmenorrhea, intestinal, hepatic, and renal colics, spasm of the bladder, and nocturnal incontinence of urine.

As a secretory depressor in night-sweats, diarrhea, galactorrhea, leukorrhea, and ptyalism.

As a stimulant in shock, collapse, pneumonia, and cardiac failure.

As a mydriatic in iritis and keratitis, but not in glaucoma.

Administration.-As a stimulant use atropin. For anodyne and antispasmodic effect on the bladder, it is best given in the form of a rectal suppository. To check the secretion of milk apply the ointment to the breasts. For asthma the inhalation of smoke from the burning leaves is best. Children bear belladonna well.

As an antidote atropin is much employed in poisoning by opium, physostigma, pilocarpin, chloral, chloroform, and prussic acid.

Pretoxic Signs.-Dryness of the mouth and throat, dilatation of the pupils, and a scarlet cutaneous eruption.

Toxicology.-Dry throat, dilated pupils, flushed 
face, rapid pulse, giddiness, delirium, blindness, and coma.

Management.-Empty and wash out the stomach. Give a solution of tannic acid. Stimulate with strong coffee enemata, strychnin, and ammonia. The physiological antidotes of belladonna are morphin, eserin, and pilocarpin.

\section{BENZOIN COMPOUNDS}

Derivation.-A balsamic resin from Styrax Benzoin. Constituents.-Benzoic and cinnamic acids.

Preparations.

Benzoinated Lard.

Tincture.-Dose, $15-60$ minims.

Compound Tincture (Friar's Balsam).-Dose, I560 minims.

Benzoic Acid.-Dose, 5-I 5 grains.

Ammonium Benzoate.-Dose, 5-15 grains.

Sodium Benzoate.-Dose, 5-60 grains.

Lithium Benzoate.-Dose, 5-20 grains.

Physiologic Action.

Antiseptic.-Benzoic acid is a strong antiseptic, a I : 1000 solution will inhibit the growth of bacteria. This drug is excreted by the urine as hippuric acid, and renders this fluid acid and slightly antiseptic.

Expectorant.-The drug is also partially excreted by the bronchial mucous membrane, and thus stimulates the secretion.

Antipyretic.-The benzoates will cause the fall of pyretic temperature.

Therapeutic Indications.

As an antiseptic in various forms of cystitis, gonorrhea, phosphaturia, and urinary incontinence. 
As an expectorant in chronic bronchitis.

Administration.-The taste of the benzoates may be covered with the spirit of chloroform. Benzoin is much used as an inhalant in respiratory diseases by placing some of the tincture on boiling water, when the arising vapor will be charged with the drug.

\section{BISMUTH COMPOUNDS}

\section{BISMUTH SALICYLATE}

Is a white, soft powder, insoluble in water and alcohol.

Other bismuth compounds are: Bismuth Benzoate, 5-15 grains; Bismuth Betanaphtolate, 5-I5 grains; Bismuth Nitrate, $1-5$ grains ; Bismuth and Ammonium Citrate, $2-5$ grains.

Physiologic Action.

Astringent.-Bismuth preparations have a slight astringent action externally and in the gastro-intestinal tract.

Antiseptic.-They are also slightly antiseptic in the intestines.

Therapeutic Indications.

As an astringent and antiseptic in skin diseases, wounds, and ulcers. In diarrhea, typhoid fever, gastric ulcer, vomiting, and gastric fermentation.

Toxicology.-Stomatitis, nausea, vomiting, diarrhea, and tendency to formation of ulcers in the mouth may follow toxic doses of bismuth preparations.

Management.-Give tannin or gallic acid.

BISMUTH SUBCARBONATE

This is a white, insoluble powder, without odor or taste. 
Dosage.-5-30 grains.

\section{BISMUTH SUBGALLATE}

This preparation is also known as dermatol, and is a yellow, insoluble powder, without odor or taste.

Dosage. $-4-8$ grains.

\section{BISMUTH SUBNITRATE}

A white, insoluble, odorless and tasteless powder. Dosage.-5-40 grains.

\section{BORIC ACID}

Properties.-A white, odorless crystal or scale, soluble in water (25 parts), glycerin (IO parts), alcohol (I 5 parts).

Synonym.-Boracic Acid.

Dosage.-5-I 5 grains.

Preparations.

Glycerite of Boroglycerin.-Also called Boroglycerid.

Sodium Borate (Borax or Biborate of Sodium) is soluble in water ( 16 parts), glycerin ( 1 part). Dose 5-30 grains.

Physiologic Action.

Antiseptic.-Boric acid is a mild antiseptic, both externally and internally. Large doses retard digestion. Boric acid renders alkaline urine acid.

Therapeutic Indications.

Boric acid is used very extensively as a mild antiseptic in diseases of the eye, ear, nose, throat, and for washing out the bladder. It is also employed to a limited extent in dressing wounds and ulcers. 
Internally it is used in fermentative dyspepsia and in cystitis of the ammoniacal type.

Borax is used the same as boric acid, and also in epilepsy.

\section{BUCHU (*)}

\section{CACTUS (*)}

\section{CAFFEIN}

Derivation.-An alkaloid derived from the common coffee berry, Caffea Arabica.

Synonyms. - Thein, Guaranin.

Properties.-A white, slender crystal, soluble in water (80 parts), alcohol (35 parts), ether (550 parts).

Dosage. $-\frac{1}{2}-5$ grains.

Preparations.

Citrated Caffein.-Dose, 2-Io grains.

Caffein Hydrobromate.-Dose, $\frac{1}{2}-2$ grains.

Caffein and Sodium Benzoate.-Dose, 2-1o grains. Caffein and Sodium Salicylate.-Dose, 2-10 grains.

Physiologic Action.

Cardiac Stimulant.-Caffein produces a rapid and forcible heart action.

Cerebral Excitant.-Caffein causes an increase of intellection and rapidity of thought. The memory is cleared. Nervousness often follows the excessive use of coffee and tea.

Diuretic.-This drug increases the blood supply to the kidneys and stimulates the renal cells, thus causing an increase in the flow of urine.

Therapeutic Indications.

As a cardiac stimulant in organic heart disease, 
palpitation due to the excessive use of tobacco, and in the cardiac depression of pneumonia, shock, and acute diseases.

As a diuretic in renal and cardiac dropsy.

As a cerebral excitant in temporary mental or intellectual depression.

Administration.-A strong, black infusion of coffee is most excellent as a stimulant when given as an enema.

Pretoxic Signs.-Headache, restlessness, insomnia, and tremor.

Toxicology.-Caffein in toxic doses produces insomnia, giddiness, trembling, profuse urination, colic, rapid, feeble pulse, and collapse.

Management.-Empty the stomach, give tannic acid, and stimulate.

\section{CALAMUS (*)}

\section{CALCIUM COMPOUNDS}

\section{CALCIUM BROMID}

This salt has the same action and uses as potassium bromid, except that the calcium salt is not depressing to the heart.

Dosage.-5-20 grains.

\section{CALCIUM CARBONATE}

Synonym.-Chalk.

Preparations.

Prepared Chalk.-Dose, 10-60 grains.

Compound Chalk Powder.-Dose, 5-30 grains.

Chalk Mixture.-Dose, I-4 drams.

Mercury and Chalk (Gray Powder).-Dose, I-8 grains. 
Physiologic Action.

Antacid.-Chalk preparations, when given internally, partially overcome the acidity of the gastric contents.

Astringent.-Both externally and internally chalk tends to dry up and lessen secretions.

Therapeutic Indications.

As an antacid in acid dyspepsia and gastric fermentation.

As an astringent it is used externally in moist eczema. Internally it is employed in diarrhea.

\section{CALCIUM CHLORID}

This salt, when applied externally or given internally, will relieve itching (antipruritic).

It is also useful in preventing hemorrhage in bleeders, and is much employed before operations on persons who are jaundiced and very liable to bleed profusely when cut.

Dosage.-5-20 grains.

\section{CALCIUM GLYCERINOPHOSPHATE}

(See Phosphorus)

\section{CALCIUM HYPOPHOSPHITE}

(See Phosphorus)

\section{CALCIUM OXID OR CALX}

Synonyms.-Calx, Lime, Quicklime.

Preparations.

Lime-water (Liquor Calcis).-Dose, I-4 ounces. The action and use of lime-water is the same as that of chalk, as an antacid and astringent. 
Syrup of Lime.-Dose, 20 minims.

Liniment (Carron Oil) consists of equal parts of lime-water and linseed oil. It is much used in the treatment of burns.

Chlorinated Lime (Calcium or Lime Hypochlorite, bleaching powder, but not Calcium Chlorid).- This preparation is a powerful oxidizing agent, and is much used as a disinfectant.

\section{CALCIUM SULPHID}

Synonyms.-Sulphurated Lime, Calx Sulphurata.

This preparation is much employed in averting pustular collections, as boils, carbuncles, and small abscesses.

Dosage. $-\frac{1}{10}-\frac{1}{2}$ grain.

\section{CALOMEL}

(See Mercury Compounds)

\section{CALUMBBA $(*)$}

\section{CAMPHOR}

Derivation.-A stearopten obtained from Camphora Cinnamomum.

Synonyms.-Camphora, Gum Camphor.

Properties.-It is soluble in water (I000 parts), alcohol (I part), olive oil (4 parts), turpentine (3 parts).

Incompatibilities.-Chloral, menthol, carbolic acid, salol, and salicylic acid.

Dosage. $-2-5$ grains.

Preparations.

Water-Dose, 1-I ounce.

Spirit.-Dose, I 5-6o minims. 
Liniment (Camphorated Oil).-20 per cent. strength.

Cerate. -2 per cent. strength.

Camphor Monobromate.-Dose, 2-5 grains.

Camphoric Acid.-Dose, Io-20 grains.

Physiologic Action.

Antipruritic.-Applied externally camphor relieves itching.

Carminative.-Internally the drug stimulates the gastro-intestinal walls, causing the expulsion of flatus.

Cardiac Stimulant.-Reflexly from the stomach, and by its action directly upon the heart, camphor increases the heart's power.

Nerve Sedative.-In small doses this drug quiets the cerebrospinal centers.

Diaphoretic.-Camphor, being partially eliminated by the skin, increases the excretion of sweat.

Therapeutic Indications.

As an antipruritic in skin diseases and diabetic pru- . ritis.

As a carminative in intestinal flatulence, spasmodic colic, and diarrhea.

As a nerve sedative and antispasmodic in hysteria, nervousness, dysmenorrhea, hiccough, headache, and asthma.

As a cardiac stimulant in the transient heart depression of the acute fevers and in shock.

As a diaphoretic in "breaking up colds."

Camphoric acid is employed in checking the nightsweats of tuberculosis.

Administration.-As a rapid heart stimulant camphor may be given hypodermically in a solution with 
sterile olive oil, using Io drops of a Io per cent. solution.

Toxicology.-Large doses of camphor cause giddiness, fatigue, rapid, weak pulse, headache, vertigo, delirium, and convulsions.

Management.-Empty the stomach and stimulate with alcohol, ether, or strychnin.

\section{CANNABIS INDICA}

Derivation.-The flowering tops of the female plant. Synonym.-Indian Hemp.

Incompatibilities.-Water precipitates the resin of the liquid preparations.

Dosage.-2-5 grains.

Preparations.

Extract.-Dose, $\frac{1}{8}-\mathrm{I}$ grain.

Fluidextract.-Dose, 2-5 minims.

Tincture.-Dose, 5-20 minims.

Cannabin Tannate.-Dose, I-IO grains.

Physiologic Action.

Narcotic.-This drug causes great exhilaration, intoxication, and delirium, followed by prolonged and deep sleep.

Sedative.-In small doses it produces quietude and composure and relieves pain.

Therapeutic Indications.

As a sedative in chorea, hysteria, insanity, and delirium tremens.

As an analgesic in headache, neuralgia, gout, gastralgia, gonorrhea, and dysmenorrhea.

Toxicology.-Although this drug may cause pronounced symptoms, yet it very seldom proves fatal. 


\section{CANTHARIDES (*)}

\section{CAPSICUM}

Derivation.-The fruit of Fastigiatum Capsicum. Synonyms.-Cayenne Pepper, Red Pepper. Dosage.-I- 5 grains.

Preparations.

Fluidextract.-Dose, I-5 minims.

Tincture.-Dose, I 5-30 minims.

Plaster.

Oleoresin.-Dose, $\frac{1}{4}-\mathrm{I}$ grain.

Physiologic Action.

Rubefacient.-Applied externally the drug causes a burning sensation, with reddening of the surface.

Carminative.-In the stomach, capsicum produces a sensation of heat and an increase of the gastric secretion, and aids in the expulsion of gas.

Therapeutic Indications.

As a rubefacient in rheumatism, lumbago, neuralgia, and abdominal colic.

As a carminative and stomachic in flatulent dyspepsia, colic, alcoholic gastritis, atony of the stomach, and diarrhea.

\section{CARBOLIC ACID}

Properties.-It is a colorless, crystalline mass, with an aromatic odor and a burning, sweet taste.

Synonyms.-Phenol, Phenic Acid, Phenyl Alcohol.

Preparations.

Glycerite.-Dose, 2-5 minims.

Ointment. -5 per cent. strength.

Physiologic Action. 
Antiseptic.-Carbolic acid is a strong and efficient antiseptic.

Anesthetic.-Applied to the skin carbolic acid causes a blanching of the surface, followed by numbness or anesthesia. Prolonged action produces a destruction of tissue (caustic).

Cardiac and Respiratory Depressant.-This drug in large doses paralyzes both of these vital functions.

Gastric Sedative.-In very minute doses carbolic acid, by its local action, quiets the stomach.

Renal Irritant.-The drug is excreted by the kidneys and is very irritating to these organs, often causing urinary suppression.

Therapeutic Indications.

As an antiseptic it is extensively used in surgery for many purposes : sterilizing instruments, dressing wounds, and irrigating cavities.

As a gastric scdative and antiseptic in persistent vomiting, flatulent dyspepsia, diarrhea, and fermentative gastritis.

As an anesthetic in toothache and itching of the skin.

Toxicology.-When applied locally in weak solution toxic symptoms often follow the use of this drug. Toxic signs are vomiting, diarrhea, colic, dark-colored urine, cold, moist skin, rapid, feeble pulse, convulsions, and collapse. Gangrene has followed the local use of a $\mathrm{I}: 20$ solution.

Management.-Do not use emetics if there is reason to believe that the gastric mucous membrane is injured. Give antidotes, as magnesium sulphate (Epsom Salt), whisky, and white of egg or milk. Stimulate with whisky, strychnin, and digitalis. Apply heat externally. 


\section{CARBON}

Synonyms.-Carbo, Charcoal.

\section{Preparations.}

Wood Charcoal (Carbo Ligni).-Dose, Io-6o grains.

Animal or Bone Charcoal (Carbo Animalis).Dose, ro-6o grains.

Physiologic Action.

Deodorant.-On account of its great absorbing power, charcoal frees substances of ill odors. It is used externally and internally.

Therapeutic Indications.

Externally it is used as an applicant to putrid ulcers and foul-smelling wounds, which it will soon rid of their odor. It is used in the form of a poultice.

Internally it is employed in acid gastric fermentation, dyspepsia, diarrhea, and as an antidote to poisoning by opium and nux vomica.

CARAWAY $\left.{ }^{*}\right)$

\section{CARDAMOM (*)}

\section{CARYOPHYLLUS $\left(^{*}\right)$}

\section{CASCARA SAGRADA}

Derivation.-The bark of Rhammus Purshiana.

Constituent.-Cascarin, a glucosid.

Dosage.-I 5-30 grains.

Preparations.

Extract.-Dose, I-6 grains.

Fluidextract.-Dose, i 5-60 minims.

Cascarin (Resinoid).-Dose, $\frac{1}{4}-\mathrm{I}$ grain.

Action and Uses.

Cascara when taken internally acts as a tonic to 
the intestinal walls and produces normal bowel movements.

- It is much employed in chronic constipation. The dose may be gradually decreased in size and frequency and still bring about the desired action. This property makes cascara a most valuable drug, not only for the relief but for the cure of constipation. The objection to the use of the fluidextract is its intense bitterness.

\section{CASCARILLA $(*)$}

\section{CASTOR OIL}

Derivation.-A fixed oil expressed from the seeds or beans of Ricinus Communis.

Synonym.-Oleum Ricini.

Dosage.-I dram to 2 ounces.

Physiologic Action.

Cathartic.-Castor oil produces soft movements of the bowels in about five hours. Its action is non. irritating and unattended, as a rule, by griping, but is followed by constipation.

Galactagogue.-Applied locally to the breasts in the form of the leaves or given internally as a fluidextract (unofficial) it produces an increase in the flow of milk.

Therapeutic Indications.

As a cathartic whenever it is necessary to move the bowels thoroughly with a period of rest following, as in diarrhea due to the presence of irritating material in the intestines. It is also used in the beginning of acute fevers, as pncumonia, typhoid, and scarlet fevers. Castor oil should not be employed in the treatment of chronic constipation. 
As a galactagoguc, see under Physiologic Action.

Administration.-Owing to its disagreeable taste it is difficult to administer this drug to many persons. To overcome this, adults may take the drug in capsules, or its taste can be disguised by mixing it with equal parts of glycerin and adding a few drops of oil of peppermint, wintergreen, or lemon-juice. Castor oil given to pregnant women may stimulate the uterine pains and cause a premature delivery. A drop or two of laudanum may be added to the oil to prevent griping.

CATECHU (*)

CAULOPHYLLUM $(*)$

\section{CERIUM OXALATE $(*)$}

\section{CHLORAL HYDRATE}

Properties.-It is a colorless crystal or crust with a penetrating, sickening odor and an acrid taste. It is soluble in water (I part), alcohol (freely).

Incompatibilities.-Alkalies, camphor and menthol.

Dosage.-5-20 grains.

Physiologic Action.

Hypnotic.-Taken in medicinal doses chloral produces general quietude, followed by placid, natural sleep.

Cardiac Depressor.-Chloral in large doses depresses the heart's action, causing a feeble, irregular pulse.

Therapeutic Indications.

The chief use of chloral is as a sleep producer in cases where pain is not a prominent symptom, as in 
nervous insomnia. As an antispasmodic it is much used in delirium tremens, convulsions, strychninpoisoning, chorea, asthma, puerperal eclampsia, and hiccoughs.

Administration.-Great care must be taken in giving chloral to patients with heart weakness. In insomnia accompanied by pain if a small dose of morphin be given with the chloral most satisfactory results will be obtained.

Toxicology.-Large doses of chloral cause a slow, feeble, irregular pulse, cold and cyanotic skin, slow, shallow respirations, deep sleep, and coma.

Management.-Empty and wash the stomach. Give antidotes, as cocain, inhalations of amyl nitrite, strychnin, and oxygen. Apply heat externally. Enemata of strong black coffee are useful.

\section{CHLORALAMID (*)}

\section{CHLORALOSE $(*)$}

\section{CHLOROFORM}

Derivation.-A liquid derived from the action of alcohol on chlorinated lime.

Properties.-A clear, colorless, volatile liquid with a sweet taste and an agreeable ethereal odor.

Preparations.

Water.-Dose, I-8 drams.

Spirit.-Dose, I 5-60 minims.

Liniment.

Emulsion.-Dose, I-2 drams.

Physiologic Action.

Rubefacient.-When applied externally chloroform 
causes a burning sensation, followed by redness of the surface and numbness.

Stomachic.-In the stomach the drug produces a sensation of warmth and an increase of the gastric secretion.

Anesthetic.-Given by inhalation chloroform soon causes a loss of consciousness, feeling, and reflexes.

Cardiorespiratory Depressant.-This drug in excess depresses and, later, paralyzes the two great centers of cardiac and respiratory functions.

Antiseptic.-Chloroform is an antiseptic and preservative of much value.

Therapeutic Indications.

As a rubefacient and anodyne it enters largely into the treatment of neuralgias, rheumatism, lumbago, sciatica, and cramps, being used for this purpose chiefly as a liniment.

As a gastro-intestinal stimulant it is much employed in flatulence and colic.

As an antispasmodic it is valuable in convulsions, hysteria, hiccough, eclampsia, epileptic seizures. chiefly employed by inhalation.

As an anesthetic in surgical procedures.

As an antiseptic it is chiefly used to prevent organic liquids from decomposing.

It is highly praised as a cure for tapeworm.

Toxicology.-The pupils, which are first contracted, dilate widely, reflexes are lost, the pulse becomes rapid and feeble, muscular relaxation is complete, and the breathing stertorous.

Management.-Stimulate with amyl nitrite, atropin, strychnin, and digitalis. Apply artificial respiration 
and make rhythmic traction on the tongue. Give inhalations of oxygen and apply the faradic current.

\section{CHRYSAROBIN $(*)$}

\section{CIMICIFUGA $(*)$}

\section{CINCHONA}

Derivation.-The bark of Cinchona Calisaya.

Synonyms.-Calisaya, Peruvian Bark.

Constituents.-Quinin, cinchonin, quinidin, cinchonidin, and cinchotannic acid.

Incompatibilities.-Metallic salts, lime-water, and tartar emetic.

Dosage.-5-15 grains.

Preparations.

Extract.-Dose, 5-30 grains.

Fluidextract.-Dose, 5-60 minims.

Tincture.-Dose, $\frac{1}{2}-2$ drams.

Infusion.-Dose, 2-8 drams.

Quinin.-Dose, $\frac{1}{2}-15$ grains.

Quinin Arsenate.-Dose, $\frac{1}{16}-\frac{1}{8}$ grain.

Quinin Bisulphate.-Dose, $\frac{1}{2}-20$ grains.

Quinin Hydrobromate.-Dose, $\frac{1}{2}-20$ grains.

Quinin Hydrochlorate.-Dose, $\frac{1}{2}-20$ grains.

Quinin Salicylate.-Dose, 2-1 5 grains.

Quinin Sulphate.-1-20 grains.

Quinin Tannate.-Dose, $\frac{1}{2}-15$ grains.

Cinchonidin.-Dose, I-20 grains.

Cinchonidin Salicylate.-Dose, I-IO grains.

Cinchonidin Sulphate.-Dose, I-20 grains.

Physiologic Action. 
Stomachic.-Cinchona stimulates the flow of gastric juice, increases the appetite, and aids digestion.

Antiperiodic.-This drug prevents the return of periodic symptoms of malaria by its action on the plasmodia circulating in the blood.

Cerebral Excitant.-Quinin produces cerebral congestion and stimulation.

Spinal Depressant.-Quinin acts as a depressant to the spinal cord and lessens reflex action.

Cardiac Stimulant.-Cinchona in small doses increases the heart's action.

Antipyretic.-Quinin produces a fall of body temperature when given in febrile conditions.

Ecbolic.-In some pregnant women quinin will stimulate uterine contractions.

Therapeutic Indications.

As a stomachic in convalescence, atonic dyspepsia, loss of appetite, and anemia.

As an antiperiodic in all malarial affections.

As an antipyretic in pneumonia, influenza, rheumatism, and other febrile disorders.

Quinin has been found of great value in the treatment of whooping-cough.

Administration.-In malaria, quinin should be given in large doses some time before the expected paroxysm, or it may be used in moderate doses during the entire twenty-four hours before the attack. Persons going to malarial districts should take quinin in small doses as a prophylactic. If a cathartic be first given the action of the quinin is greatly augmented. Quinin should be given in powder form in capsules or konseals and not in pilular or tablet form, for if 
these latter are not recently made their action is uncertain.

Pretoxic Signs.-Ringing or buzzing in the ears, fulness of the head, and slight deafness.

Toxicology.-The toxic symptoms of poisoning by quinin or cinchona are grouped as cinchonism: deafness, blindness, tinnitus aurium, skin eruptions, slow pulse, and coma.

\section{CINNAMON $(*)$}

\section{CITRIC ACID (*)}

\section{COCA}

Derivation.-The leaves of Coca Erythroxylon.

Synonyms.-Cuca, Erythroxylon.

Constituent.-Cocain, an alkaloid.

Dosage. - 5-30 grains.

\section{Preparations.}

Fluidextract.-Dose, I-4 drams.

Cocain Hydrochlorate.-Dose, $\frac{1}{4-2}$ grains.

Physiologic Action.

Anesthetic.-Cocain, when applied to the mucous membrane, the broken skin, or injected subcutaneously, produces numbness of the parts with a blanching of the surface, due to the constriction of the blood-vessels.

Cerebrospinal stimulant.-When given in moderate doses coca produces a sense of exhilaration, increases the mental powers, and causes insomnia.

Cardiac Stimulant. - In small doses this drug acts as a heart stimulant.

Mydriatic.-Applied to the conjunctiva, cocain soon produces marked dilatation of the pupils. 
Therapeutic Indications.

As a local anesthetic in minor operations and in the relief of pruritus, toothache, painful ulcers, sore gums, and in vomiting.

As a cerebrospinal stimulant in melancholia, neurasthenia, and hysteria.

As a mydriatic in iritis and keratitis.

Administration.-As a subcutaneous injection cocain may be employed as a I to Io per cent. solution, and not more than $\frac{3}{4}$ grain should be used.

Toxicology.-Cocain should be used as an anesthetic with great caution, as it may produce grave toxic symptoms, as uncontrollable laughter followed by mental depression, dryness of the throat, nausea and vomiting, dilated pupils, feeble, irregular pulse, cyanosis, fainting, and collapse.

Management.-Wash out the stomach. Give stimulants, as strychnin and aromatic spirit of ammonia. Begin artificial respiration early and give inhalations of oxygen.

\section{CODEIN}

\section{(See Opium)}

\section{COD-LIVER OIL}

Derivation.-A fixed oil derived from the fresh livers of the Gadus Morrhua or the codfish.

Synonym.-Oleum Morrhuæ.

Dosage.-I-4 drams.

Preparation.

Emulsion.-Dose, I-8 drams.

Physiologic Action.

Alterative.-Taken internally, cod-liver oil so affects 
metabolic processes that the general nutrition of the body is improved, the resistance of the person is increased, and adipose tissue is deposited. Part of this action is due to its oily properties, and partly to certain extractives found in the drug.

Therapeutic Indications.

Cod-liver oil is employed in all conditions accompanied by failure of the general nutrition of the body, as in tuberculosis, scrofula, diabetes, chronic bronchial affections, anemia, rickets, syphilis, neurasthenia, and chronic rheumatism.

Administration.-The clear oil is the best form for giving this drug, but its taste to many individuals is revolting. Children learn to take it readily. It may be given in capsules or mixed with glycerin and a few drops of oil of wintergreen. If a little salt be added to the oil, regurgitation may be prevented.

\section{COLCHICUM}

Derivation.-The corm and seed of Colchicum Autumnale.

Synonym.-Meadow Saffron.

Constituent.-Colchicin.

Dosage.-I- -5 grains.

Preparations.

Extract of the Root.-Dose, $\frac{1}{2}-2$ grains.

Fhiidextract of the Root.-Dose, 2-8 minims.

Fhidextract of the Secds.-Dose, 3-10 minims.

Tincture of the Sceds.-Dose, 5-30 minims.

Wine of the Root.-Dose, 5-20 minims.

Wine of the Seeds.-Dose, 20-60 minims.

Colchicin.-Dose, $\frac{1}{120} \frac{1}{30}$ grain. 
Physiologic Action.

Irritant.-Applied externally, colchicum causes burning and redness, and if the action continues pustules form.

Gastro-intestinal Irritant.-In very small doses the drug stimulates the gastric, intestinal, and biliary secretions, but in larger doses it acts as an irritant, producing nausea, vomiting, colic, and purging.

Cardiorespiratory Depressant.-Colchicum acts on the heart similarly to aconite, producing a slow, soft pulse. Respirations are first slowed, but later becomc rapid and shallow.

Antipodagric. - The chief use of colchicum is in gout. How its action is brought about in this disease little is known, but it soon lessens the pain and joint symptoms.

Therapeutic Indications.

As mentioned above, colchicum is chiefly employed in the treatment of podagra (gout). It is also useful in chronic rheumatism

Toxicology.-Large doses produce vomiting, purging (which may become bloody), rapid, feeble pulse, shallow respirations, most intense colic, cold, moist skin, and collapse.

Management.-Empty the stomach by giving apomorphin or emetics, as mustard-or salt-water. Tannic acid is an excellent antidote. Stimulate with enemata of strong coffee, strychnin, and atropin. 


\section{CONVALLARIA}

Derivation.-The rhizomes and roots of Convallaria Majalis.

Synonym.-Lily of the Valley.

Dosage. - I-5 grains.

Preparations.

Fluidextract.-Dose, 5-20 minims.

Convallamarin.-Dose, $\frac{1}{2}-1$ grain.

Physiologic Action.

Cardiac Stimulant.-Convallaria produces a slow, powerful heart action, and is said to act especially on the right side of the heart.

Diuretic.-The use of convallaria is followed by an increase of the urinary excretion.

Comparative Action.-Compared to digitalis, convallaria is not so energetic, produces less slowing of the heart, does not cause so great vasoconstriction, and acts especially on the right heart.

Therapeutic Indications.

It is used in cardiac disease as is digitalis, for which it may be substituted.

COPAIBA $(*)$

COPPER COMPOUNDS $(*)$

COTARNIN HYDROCHLORATE

(See Part IV.)

CREOLIN (*)

CREOSOTE AND COMPOUNDS

Derivation.-Creosote is an oily liquid derived from the distillation of beech-wood. 
Properties.-It is a colorless liquid with an empyreumatic odor and a burning taste. It is soluble in water ( 150 parts), alcohol (freely).

Constituents.-Guaiacol and Creosol.

Dosage. $-\frac{1}{2}-2$ minims.

Preparations.

Water.-Dose, 1-2 drams.

Creosote Carbonate (Creosotal).-Dose, 5-20 minims.

Physiologic Action.

The action of this drug is very much like that of Carbolic Acid (which see).

Locally it is an anesthetic and parasiticide. Internally it is an antiseptic and gastric sedative.

Therapeutic Indications.

The chief use of creosote is in pulmonary tuberculosis, when it acts as an antiseptic and increases the nutrition. It is also much employed as an intestinal antiseptic in typhoid fever and diarrhea. In vomiting it is very useful.

Creosote carbonate is less irritating than creosote, and is extensively employed in tuberculosis, pneumonia, and typhoid fever.

Administration.-As creosote is more or less of a gastric irritant, it is best given in capsules or in the form of an emulsion. Creosote is much used as an inhalant, and as such may be given on a mask in the following combination: Chloroform, ether, and creosote, of each I dram; then add 2 drams of alcohol and I dram of the oil of eucalyptus. Sprinkle about 20 drops of this on the mask or inhaler.

Toxicology.-See under Carbolic Acid. 


\section{CROTON CHLORAL (*)}

\section{CROTON OIL}

Derivation.-A fixed oil cxpressed from the seeds of Croton Tiglium.

Synonym.-Oleum Tiglii.

Dosage. $-\frac{1}{4}-1$ minim.

Physiologic Action.

Rubefacient and Pustulant.-Applied externally croton oil produces great irritation and redness, soon followed by the formation of pustules.

Drastic Cathartic.-Taken internally the drug causes considerable irritation, with profuse and watery purgation. It may bring about a severe gastroenteritis.

Therapeutic Indications.

As a countcr-irritant in pleurisy, arthritis, bronchitis, and neuralgia.

As a cathartic when it is desirable to get rapid and profuse bowel movements, or for use in unconscious patients, as in apoplexy, mania, and coma.

Administration.--If the patient be unconscious I drop of croton oil may be mixed with a little butter or glycerin and placed on the base of the tongue. It should never be given to patients suffering with inflammation of the stomach, intestines, or peritoneum. This potent drug should not be administered to children or pregnant women. Not more than 2 drops should be given as a single dose.

Toxicology.-Nausea, vomiting, great purging, severe abdominal pain, followed by collapse.

Management.-Empty the stomach. Give demul- 
cents, as mucilage, starch paste, oils, flaxseed-tea, and white of egg. Give opium for the pain. Stimulate with ammonia, brandy, and strychnin.

\section{CUBEBS $\left(^{*}\right)$}

\section{CUSSO $(*)$}

\section{DIGITALIS}

Derivation.-The leaves of Digitalis Purpurea, collected in the second year.

Synonym.-Foxglove.

Constituents.-Digitalin, digitoxin, digitonin, and digitalein-all are glucosids.

Incompatibilities. - Acids, alkalies, tannin, cinchona, and iron and lead salts.

Dosage.-I- 3 grains.

Preparations.

Extract.-Dose, $\frac{1}{4}-\mathrm{I}$ grain.

Fliidextract.-Dose, $\frac{1}{2}-3$ minims.

Tincture.-Dose, 5-30 minims.

Infusion.-Dose, I-4 drams.

Digitalin.-Dose, $\frac{1}{50}$ grain.

Physiologic Action.

Gastro-intestinal Irritant.-Digitalis disorders the stomach and causes nausea and vomiting in many persons.

Cardiac Stimulant.-Upon the heart digitalis acts as a stimulant. The heart action becomes slower, but more forcible.

Vasoconstrictor.-By its stimulating action on the vasomotor center of the brain, digitalis causes a 
marked constriction of the blood-vessels and raises the general blood-pressure.

Diuretic.-By the combined action of this drug on the heart and vessels, together with its local action on the kidneys, the amount of urine excreted is greatly increased.

Therapeutic Indications.

As a cardiac stimulant in chronic endocarditis with involvement of any of the valves. In tachycardia, palpitation, and irritable heart it is useful. In heart depression, occurring in the course of typhoid fever, pneumonia, shock, drug poisoning, acute fevers, and alcoholism.

As a diuretic in dropsy of renal or cardiac origin.

Digitalis is also employed in exophthalmic goiter, menorrhagia, and hemorrhage.

Administration.-As digitalis easily disorders the stomach, it may be given to susceptible persons as an enema. As a heart stimulant use the tincture, and for diuretic effect the infusion is preferable. Fever prevents the slowing action of digitalis on the heart. Children bear digitalis badly. As it is often desirable to combine digitalis with iron, the black mixture resulting from such a combination may be readily cleared by adding a little dilute phosphoric acid.

Digitalis slowly accumulates in the system, and on this account the drug should be stopped occasionally.

Pretoxic Signs.-A slow pulse becoming rapid, and a decrease in the flow of urine.

Toxicology.-Large doses produce a rapid, tumultuous heart, nausea, vomiting, purging, headache, and, later, collapse. 
Management.-Empty the stomach and wash repeatedly. Give tannin as an antidote. Stimulate with ammonia, brandy, strychnin, and atropin. Keep the patient in the reclining position. This is important.

\section{ELATERIUM $(*)$}

\section{ERGOT}

Derivation.-Ergot is produced by the action of a fungus on the grain of common rye.

Synonyms.-Spurred Rye, Ergot of Rye, Ergota.

Constituents.-Ergotin and Cornutin.

Dosage.-30-60 grains.

Preparations.

Extract.-Dose, 5-I 5 grains.

Fluidextract.-Dose, $\frac{1}{2}-2$ drams.

Wine.-Dose, I-4 drams.

Ergotin (pure aqueous extract).-Dose, I-IO grains.

Physiologic Action.

Hemostatic.-Ergot given internally causes a general contraction of the blood-vessels and a rise of blood-pressure.

Oxytocic.-Ergot produces, by its action on the muscles of the uterus, great contraction of that organ.

Therapeutic Indications.

As a hemostatic in cerebral and spinal congestion, in post-partum hemorrhage, in hematuria, hemoptysis, hematemesis, and epistaxis; to check secretions in profuse sweating, in diarrhea, diabetes insipidus, and in excessive secretion of milk.

As a uterine stimulant in post-partum hemorrhage, subinvolution, and menorrhagia. 
This drug is of value in chronic alcoholism and delirium tremens.

Toxicology.-Acute Poisoning.-Nausea, vomiting, diarrhea, slow pulse, dizziness, giddiness, tremor, disturbed vision, areas of numbness, subnormal temperature, and collapse.

Management.-Empty the stomach with mustard or apomorphin. Give tannin. Stimulate with brandy. Give inhalations of amyl nitrite and hypodermic injections of nitroglycerin.

Chronic Poisoning.-Headache, vertigo, drowsiness, itching, photophobia, spasms of the muscles, coldness and numbness of the fingers and toes, followed by dry gangrene.

\section{ERIGERON (*)}

\section{ERIODICTYON (*)}

\section{ETHER}

Derivation.-A liquid derived from the distillation of alcohol with sulphuric acid.

Synonyms.-Sulphuric Ether, Ethyl Oxid.

Properties.-A clear, volatile liquid with a sweet, burning taste and a characteristic odor.

Dosage. - 5-60 minims.

Preparations.

Spirit.-Dose, 1 5-60 minims.

Compound Spirit (Hoffman's Anodyne).-Dose, 5-60 minims.

Physiologic Action.

Anesthetic.-Applied locally it produces great cold and numbness, due to its rapid evaporation. 
Given by inhalation it soon causes unconsciousness, with loss of reflexes and sensation.

Cardiac Stimulant.-Ether rapidly produces a quick, forcible heart with an increase of blood-pressure.

Respiratory Stimulant.-Ether at first causes rapid and deep respirations.

Therapeutic Irdications.

As an anesthetic in surgical procedure.

As an antispasinodic in tetanus, hysteric spasms, convulsions, eclampsia, hiccoughs, and asthma.

As a stimulant in syncope, shock, collapse, and heart depression occurring in the acute fevers.

Externally in neuralgias, toothache, and rheumatism. Administration.-Ether, when given internally, should be employed in capsules or as a syrup.

Toxicology.-See Chloroform.

$$
\begin{aligned}
& \text { ETHYL BROMID } \\
& \text { ETHYL CHLORID }
\end{aligned}
$$

EUCAIN (*)

\section{EUCALYPTUS (*)}

EUONYMUS (*)

\section{EUPATORIUM (*)}

\section{FORMALDEHYD}

Derivation.-A colorless gas derived from the action of heat on wood alcohol.

Synonyms.-Formic Aldehyd, Formol.

Preparations.

Formalin, which is a 40 per cent. solution of formaldehyd in water. 
Physiologic Action.

Antiseptic.-This drug has great powers as an antiseptic and disinfectant. On account of its penetrating power it is most efficient.

Anhidrotic.-Applied externally, formalin soon hardens the skin and lessens sweating.

Therapeutic Indications.

As an anhidrotic for sweating feet or axillæ.

As an antiseptic for instruments, catheters, and for dressing wounds.

As a disinfectant for rooms, clothing, and furniture after contagious or infectious diseases.

As a preservative of anatomic and pathologic specimens.

\section{FRANGULA $(*)$}

\section{GALLA}

Deflnition.-Nutgalls are excrescences on the oak, caused by the puncture and eggs of a small insect.

Synonyms.-Nutgalls, Galls.

Constituents.-Tannic and gallic acids.

Incompatibilities.-Alkalies, alkaloids, metallic salts, acids, and gelatin.

Preparations.-

Tincture of Nutgalls.-Dose, I-2 drams.

Ointment of Nutgalls.-20 per cent. strength.

Tannic Acid (Tannin).-Dose, I-5 grains.

Ointment of Tannic Acid.

Glycerite of Tannic Acid.

Styptic collodion contains 20 per cent. of tannic acid.

Gallic Acid.-Dose, I-I 5 grains. 
Physiologic Action.

Astringent.-Applied externally, tannic acid coagulates albuminous discharges, stops bleeding, and astringes the tissues. Internally, it acts as an astringent, diminishing the gastric and intestinal juices.

Gallic acid, on the other hand, does not coagulate albumin, but controls hemorrhage by constricting the blood-vessels.

Therapeutic Indications.

Tannic acid is used locally to stop bleeding; to contract relaxed tissues, as in rhinitis, pharyngitis, and uvulitis; to check excessive sweating of tuberculosis or localized sweating of the feet or axillæ. It is useful as an injection in leukorrhea.

Internally it is used in diarrhea, diabetes, and renal bleeding.

Gallic acid is given in the treatment of hematuria, menorrhagia, night-sweats, diabetes, albuminuria, and diarrhea.

\section{GAULTHERIA}

Derivation.-A volatile oil distilled from the leaves of Gaultheria Procumbens.

Synonyms.-Oil of Wintergreen, Oil of Teaberry.

Dosage.-5-20 minims.

Preparation.

Spirit.-Dose, I-2 drams.

Physiologic Action.

The action and uses of the oil of wintergreen are the same as those of Salicylic Acid (which see).

Administration.-It is best given in capsules, as an emulsion, or on sugar. There is on the market a syn- 
thetic oil of wintergreen made from carbolic acid derivatives and called methyl salicylate. This preparation should not be employed internally as a substitute for the natural oil.

\section{GELSEMIUM}

Derivation.-The rhizomes of Gelsemium Sempervirens.

Synonym.-Yellow Jasmine.

Constituents.-Gelsemin and Gelseminin.

Dosage.-3-10 grains.

Preparations.

Fluidextract.-Dose, 2-5 minims.

Tincture.-Dose, 10-30 minims.

Gelsemin.-Dose, $\frac{1}{120}-\frac{1}{60}$ grain.

Physiologic Action.

Spinal Depressant.-Gelsemium acts mainly on the anterior horn of the cord, depressing it, thus causing loss of reflexes and decreased muscular power. The sensory side is also affected, producing anesthesia.

Mydriatic.-This drug produces dilatation of the pupils.

Gelsemium is a cardiorespiratory depressant.

Therapeutic Indications.

As an antispasmodic in whooping-cough, asthma, and spasmodic dysmenorrhea.

As an analgesic in neuralgia, rheumatism, headache, and migraine.

Administration.-As a mydriatic gelsemin is used in a $1: 60$ solution in water.

Toxicology.-Large doses produce languor, malaise, and weakness, followed by muscular relaxation, as 
ptosis and dropping of the jaw. The pupils are widely dilated, the pulse rapid, feeble, and irregular, and the skin cold and moist.

Management.-Empty the stomach with emetics, as mustard-, salt-water, or apomorphin. Give tannic acid. Stimulate with ammonia, atropin, and strychnin.

\section{GENTIAN}

Derivation.-The roots of Gentiana Lutea.

Incompatibilities.-Iron and lead salts.

Dosage. - 5-30 grains.

Preparations.

Extract.-Dose, I-Io grains.

Fluidextract.-Dose, 5-30 minims.

Compound Tincture.-Dose, I-4 drams.

Physiologic Action.

Stomachic.-In medicinal doses gentian sharpens the appetite, increases the flow of the gastric secretions, and the motor power of the stomach and intestines.

Therapeutic Indications.

Gentian is employed whenever a bitter tonic is necessary, as in anorexia, atonic dyspepsia, hysteria, anemia, etc.

\section{GINGER}

Derivation.-The rhizomes of Zingiber Officinale.

Synonym.-Zingiber.

Dosage. - 5-I 5 grains.

Preparations.

Fluidextract.-Dose, 5-I 5 minims.

Tincture.-Dose, 10-60 minims. 
Syrup.-Dose, I-4 drams.

Oleoresin.-Dose, $\frac{1}{2}-2$ minims.

Physiologic Action.

Rubefacient.-Applied externally ginger produces aburning sensation, followed by redness of the surface.

Stomachic.-Ginger increases the flow of saliva and gastric juice and stimulates the appetite.

Carminative.-By its stimulating action on the motor power of the stomach and intestines it aids in the expulsion of gas or flatus.

Therapeutic Indications.

Ginger is used in dyspepsia, flatulence, colic, diarrhea, and dysmenorrhea. Externally it is used the same as capsicum.

\section{GLYCERIN}

Derivation.-It is a triatomic alcohol, derived from the action of superheated steam on fats.

Properties.-Glycerin is a clear, colorless, thick syrupy liquid with a sweet, warm taste.

Dosage.-5-60 minims.

Preparations.

Suppositories.

Several glycerites.

Physiologic Action.

Emollient.-Applied externally glycerin is soothing and softens the skin, and will aid in the absorption of drugs incorporated with it.

Laxative.-Given internally it produces a soft bowel movement. If given by rectum it has the same action. 
Therapeutic Indications.

Externally for chapped hands, eczema, psoriasis, and other skin diseases.

Internally it is used in constipation, either by mouth or rectum.

Glycerin is also employed as a sweetening agent for diabetics.

\section{GLYCYRRHIZA}

Derivation.-The root of Glycyrrhiza Glabra.

Synonyms.-Liquorice, Licorice.

Constituent,-Glycyrrhizin.

Dosage.-Freely.

Preparations.

Extract.-Dose, 5-6o grains.

Fluidextract.-Dose, I-2 drams.

Compound Powder contains senna, licorice, sulphur, and oil of fennel. Dose, I-2 drams.

Compound Mixture contains licorice, paregoric, wine of antimony, and spirit of nitrous ether. It is also known as Brown Mixture. Dose, I-4 drams.

Ammoniated Glycyrrhizin.-Dose, 5-I 5 grains.

Physiologic Action.

Demulcent.-Licorice allays irritation of the mouth, pharynx, and bowels.

Laxative.-Licorice is a slight laxative, causing a large, softened stool.

Vehicle.-Owing to its pleasant taste, licorice is much employed to cover the taste of unpleasant drugs.

Therapeutic Indications.

As a demulcent in stomatitis and pharyngitis. 
As a laxative it is given in the form of the compound powder, as a mild, non-griping laxative.

\section{GOSSYPII RADICIS CORTEX (*)}

GRINDELIA ROBUSTA (*)

\section{GUAIAC $\left(^{*}\right)$}

\section{GUAIACOL}

Derivation.-One of the chief constituents of Creosote.

Properties.-It is a colorless, oily liquid with an empyreumatic odor. It is soluble in alcohol and ether (freely), in water (100 parts).

Dosage. $-\frac{1}{2}-10$ minims.

Preparations.

Guaiacol Benzoate (Benzosol).-Dose, 1-10 grains. Guaiacol Carbonate (Duotal).-Dose, I-8 grains. Guaiacol Salicylate.-Dose, 5-30 grains.

Guaiacol Valerianate (Geosote).-Dose, 3-10 minims.

Physiologic Action.

The general action of guaiacol is the same as that of Creosote (which see), excepting that guaiacol has a powerful antipyretic action when applied locally. At times it reduces the temperature from five to seven degrees in a brief time.

Guaiacol carbonate is much employed as an internal antiseptic.

Therapeutic Indications.

They are the same as for creosote, but the carbonate is much used in pulmonary tuberculosis, typhoid fever, and diarrhea as an antiseptic. 
Administration.-Guaiacol should be given well diluted and its taste disguised by some vehicle or in capsules.

Locally, for reducing fever, it must be used with great caution, as it may produce sudden collapse. Thirty minims of the pure drug mixed with equal parts of glycerin are applied to the skin of the abdomen or chest by means of a camel's-hair brush, and the area covered with oiled muslin to prevent too rapid evaporation.

Applied to painful areas, as in neuralgia, rheumatism, and sciatica, guaiacol rapidly relieves the pain.

HAMAMELIS (*)

HEMATOXYLON (*)

HEROIN

(See Opium)

\section{HIRUDO}

Definition.-An aquatic worm of the class Sanguisuga Medicinalis.

Synonym.-Leech.

Therapeutic Use.

The leech is employed to abstract blood in such conditions as meningitis, mastoiditis, conjunctival ecchymosis, orchitis, and joint affections.

\section{Application.}

Have the area shaven and well cleaned, and apply the leech. If the leech does not readily take hold, then apply to the part a little fresh milk or blood. If it is desirable to have the animal attack a certain spot, 
then place the leech on a sheet of paper with a small hole in it, and have this hole over the area you wish to have attacked.

The leech will release its hold when it is engorged with blood. If, for any reason, it holds fast when you desire to remove it or if it accidently gets into the external auditory meatus, it may be readily removed by applying salt or a drop of ether to it.

If the leech-bite continues to bleed, the hemorrhage may be controlled by the application of pressure, styptic collodion, Monsel's solution, lunar caustic, or suprarenal extract.

\section{HOMATROPIN HYDROBROMATE}

Action and Uses.

This drug causes dilatation of the pupils, for which purpose it is solely employed.

Its general action is similar to atropin.

Used for the eye in a I per cent. solution.

\section{HUMULUS (*)}

\section{HYDRASTIS}

Derivation.-The rhizome and root of Hydrastis Canadensis.

Synonym.-Golden Seal, Yellow Root.

Constituents.-Two alkaloids : Hydrastin and Berberin.

Dosage. - 5-60 grains.

Preparations.

Fluidextract.-Dose, 5-60 minims.

Tincture.-Dose, $\frac{1}{2}-2$ drams.

Glycerite. 
Hydrastin.-Dose, $\frac{1}{4}-\mathrm{I}$ grain.

Hydrastinin Hydrochlorate (artificial alkaloid).Dose, $\frac{1}{4}-\frac{1}{2}$ grain.

Physiologic Action.

Astringent.-Applied to the skin or mucous membrane it is soothing and astringent, lessening the secretions

Stomachic.-Internally it increases the gastric juice and sharpens the appetite.

Cholagogue.-This drug is said to increase the flow of bile.

Cardiorespiratory Stimulant.-This action of the drug is very similar to that of nux vomica.

Styptic.-Hydrastis contracts the musculature of the vessels, causing vasoconstriction.

Oxytocic.-Hydrastis causes contraction of the muscles of the uterus.

Therapeutic Indications.

As an astringent it is used in the treatment of ulcers, wounds, local sweating, stomatitis and pharyngitis, rhinitis, leukorrhea, and gonorrhea.

As a stomachic in atonic dyspepsia and alcoholic gastritis.

As a cholagogue in catarrhal jaundice.

As a styptic in epistaxis, hemoptysis, menorrhagia, and other forms of bleeding.

\section{HYDROCHLORIC ACID}

Definition.-A liquid containing about 32 per cent. of absolute hydrochloric acid.

Synonyms.-Muriatic Acid, Acidum Hydrochloricum. 
Properties.-A clear, colorless liquid, giving off irritating vapors and having a burning, sour taste.

Incompatibilities.-Alkalies, carbonates, lead and silver salts, and potassium permanganate.

Dosage._I-IO minims, well diluted.

Preparations.

Dilute Hydrochloric Acid (Io per cent. strength).Dose, ro-30 minims.

Physiologic Action.

Caustic.-Applied externally this acid, like all of the mineral acids, causes redness and burning, and if its action is continued, destruction of tissue takes place.

Tonic.-Hydrochloric acid increases the flow of saliva, but decreases the gastric juice if it be taken before or during the meal. If administered after the meal it reinforces the action of the natural acid of the stomach and aids digestion.

Hematinic.-This acid is said to increase the number of red cells of the blood.

Therapeutic Indications.

Hydrochloric acid is useful in all diseases in which the natural acid is deficient, as in nervous dyspepsia, subacid indigestion, achylia, gastric cancer, anemia, and acute fevers, especially typhoid fever.

Administration.-This acid should always be given in a well-diluted form and after meals. If taken through a glass tube its corrosive action on the teeth may be avoided.

Toxicology.-The usual symptoms are burning of the mouth, throat, and stomach; excoriation of the lips, tongue, and mouth; vomiting of dark-colored 
material (altered blood) ; cold, moist skin ; rapid, feeble pulse, and collapse.

Management.-Do not use a stomach-tube if a strong acid has been taken, as you may start a severe hemorrhage or perforate the stomach wall. Give antidotes, as soapy water, lime-water, chalk, or saleratuswater. Give demulcents, as olive oil, cotton-seed oil, milk, white of egg, and starch solution. Give morphin for pain and stimulate if necessary.

\section{HYDROCYANIC ACID}

Definition.-It is a colorless, clear liquid, with an odor resembling bitter almonds, and is extremely poisonous. One drop of pure acid has caused death.

Synonym.-Prussic Acid.

\section{Preparations.}

Dilute Hydrocyanic Acid contains 2 per cent. of hydrocyanic acid, and is the only form used as a medicine. Dose, I-4 minims. When this solution turns brown it should not be used.

\section{Physiologic Action.}

Local Anodyne.-The dilute acid, when applied to the cutaneous surface, is rapidly absorbed and depresses the sensory end filaments, causing numbness.

Analgesic.-Taken internally the dilute acid acts as a sedative to the stomach walls. Being absorbed quickly, it acts upon the sensory nerve tissue, depressing it.

Cardiorespiratory Depressor.-Dilute prussic acid in small doses produces a slow heart; in large doses a rapid, feeble heart. The respiratory function is also depressed. 


\section{Therapeutic Indications.}

As a sedative in the hacking cough of chronic bronchitis, pulmonary tuberculosis, and in whooping-cough. In persistent vomiting, colic, and gastralgia.

As a local anodyne in all forms of itching.

Toxicology.-Poisoning by this drug is very rapid. The symptoms are salivation, giddiness, cyanosis, convulsions, fixed, staring eyes, dilated pupils, very weak and rapid pulse, and, if fatal, death will take place in a short time.

Management.-Stimulate vigorously with ammonia inhalations, electricity, and atropin. Inaugurate artificial respiration and give cold affusions.

\section{HYDROGEN PEROXID SOLUTION}

Definition.-A 3 per cent. solution of hydrogen peroxid in water.

Synonyms.-Aqua Hydrogenii Dioxidi, Oxygenized Water.

Properties.-A clear, colorless liquid with a slight acid taste, and which in the presence of organic material easily gives up its oxygen.

Dosage.-I-2 drams.

Physiologic Action.

Disinfectant and Deodorizer.-As this preparation gives up its oxygen very readily, it oxidizes organic material with which it comes in contact, thus destroying pus, dead tissue, and micro-organisms (sic).

Therapeutic Indications.

Hydrogen peroxid is employed whenever it is desired to free tissues of pus and necrotic matter, as in ulcers, fistulous tracts, abscess cavities, and diphtheritic 
patches. This drug is useful also in stopping the oozing of blood from wounds.

\section{HYOSCYAMUS}

Derivation.-The leaves and flowering tops of the second year's growth of Hyoscyamus Niger.

Synonym.-Henbane.

Constituents.-Two alkaloids: Hyoscyamin and Hyoscin.

Dosage. - 5-1 5 grains.

Preparations.

Extract.-Dose, $\frac{1}{2}-3$ grains.

Fluidextract.-Dose, 5-1 5 minims.

Tincture.-Dose, I-4 drams.

Hyoscin Hydrobromate.-Dose, $\frac{1}{400}-\frac{1}{120}$ grain.

Hyoscyamin (Amorphous).-Dose, $\frac{1}{12}-\frac{1}{6}$ minim.

Hyoscyamin (Crystal).-Dose, $\frac{1}{120}-\frac{1}{60}$ grain.

Physiologic Action.

The action of hyoscyamus is the same as that of belladonna, except that hyoscyamus has a sedative effect on the brain and cord due to the hyoscin, and hyoscyamus is more of a sedative to the bladder.

Therapeutic Indications.

This drug is much employed as a sedative in bladder troubles, as vesical spasm, cystitis, prostatitis, urethritis, and urinary incontinence.

Hyoscin is used in the insomnia and unrest of insanity, mania, and delirium tremens.

Toxicology.-See Belladonna.

ICHTHYOL

Derivation.-A substance produced by the distillation of the fossil remains of fish. 
Synonym.-Ammonium Sulpho-ichthyolate.

Properties.-It is a semiliquid, dark brown or black substance, soluble in water, and having a rank bituminous odor.

Dosage.-3-15 minims.

Physiologic Action.

Vulnerary.-This agent, when applied to wounded areas, stimulates the formation of granulation tissue and the growth of epithelia.

Alterative.-Internally ichthyol seems to alter certain morbid processes and to exert a slight antiseptic effect.

Therapeutic Indications.

Externally in the treatment of ulcers, indolent wounds, erysipelas, burns, chilblains, and nearly all skin diseases.

Internally it is said to be useful in tuberculosis, as it decreases the expectoration and lessens the fever. It should be given in capsules.

\section{INGLUVIN (*)}

\section{IODIN}

Preparations.

Tincture.-Dose, I-5 minims.

Ointment.

Compound Solution (Lugol's Solution) contains iodin, potassium iodid, and water. Dose, I-IO minims.

Physiologic Action.

Counter-irritant. - Fixternally iodin produces great irritation, with burning and redness. 
Parasiticide.-Iodin destroys the lower animal and vegetable parasites that attack the skin.

\section{Therapeutic Indications.}

Iodin is used in the various parasitic skin diseases and as an application to enlarged glands, sprained joints, and in pleurisy, pericarditis, rheumatism, and neuralgia.

It is very seldom used internally, but occasionally it will be found of great value if employed in minute doses in persistent vomiting.

Administration.- - Rather than make frequent paintings of the part, it is better to at once paint the area until it is almost black and then wait until desquamation is complete before applying the iodin again. This prevents very much local irritation.

Toxicology.-Iodin applied is absorbed and may cause the appearance of toxic signs. Salivation, tenderness of the gums, nausea, coryza, acneal eruption, anemia, various paralyses, and emaciation. These symptoms are classed as iodism.

Management.-Discontinue the use of the drug. Aid in its elimination. If it be a case of acute poisoning, wash out the stomach, give starch solution, and wash out the viscus again. Repeat this several times. Stimulate with brandy and atropin. Apply heat to the body.

\section{IODOFORM}

Properties.-A yellow crystalline powder having a penetrating odor and a sweet taste. It is soluble in ether (7 parts), alcohol ( 52 parts), glycerin (I00 parts), and insoluble in water. It contains about 95 per cent. of iodin.

Dosage.-I-3 grains. 
Preparation.

Ointment.

Action and Uses.

Iodoform is an antiseptic, disinfectant, alterative, and antitubercular. It is used as a dressing for wounds, ulcers, fistulous tracts, and burns. It is injected into tubercular abscess cavities, joints, and pleural sac.

Toxicology.-See Iodin.

\section{IPECAC}

Derivation.-The roots of Ipecacuanha Cephaëlis.

Constituents.-Emetin and Cephaëlin.

Synonym.-Ipecacuanha.

Dosage. $-\frac{1}{2}-20$ grains.

Preparations.

Fluidextract.-Dose, $\frac{1}{2}-20$ minims.

Syrup.-Dose, $\frac{1}{4}-2$ drams.

Wine.-Dose, I-6o minims.

Powder of Ipecac and Opium (Dover's Powder).Dose, 3-1 5 grains.

Tincture of Ipecac and Opium.-Dose, 3-I 5 minims.

Emetin.-Dose, $\frac{1}{12}-\frac{1}{60}$ grain.

Physiologic Action.

Irritant.-Applied to the skin or mucous membrane, ipecac produces irritation followed by redness and, if prolonged, by pustules.

Emetic.-Very small doses act as a gastric sedative, but larger doses produce nausea and vomiting by its local irritation and by its action on the vomiting center in the medulla. 
Cholagogue.-Ipecac is said to increase the flow of bile.

Diaphoretic.-This drug causes a slight increase in the excretion of the sweat.

Expectorant.-Ipecac produces a congestion of the bronchial mucous membrane and increases its secretions.

Cardiac Sedative.-In moderate doses ipecac reduces the heart's action.

Therapeutic Indication.

As an emetic in all forms of poisoning if the toxic material is in the stomach and is not of a corrosive character. It is also employed to unload the engorged bronchi in suffocative bronchitis and croup in children, and in laryngitis.

As a gastric sedative in some types of persistent vomiting, it is very useful if given in minute doses.

As an expectorant in bronchitis, laryngitis, asthma, and chronic cough.

As a diaphoretic to break up "colds," it is used in the form of Dover's Powder.

Ipecac is said to act as a specific in dysentery, but must be given in very large doses (20 grains every four hours).

\section{IRIS $(*)$}

\section{IRON COMPOUNDS}

\section{REDUCED IRON}

Synonyms.-Ferrum Reductum, Quevenne's Iron, Iron by Hydrogen.

Dosage.-I-5 grains. 
IRON ARSENATE

Synonym.-Ferri Arsenias.

Action is that of arsenic and iron combined. Dosage.- $\frac{1}{16}-\frac{1}{8}$ grain.

\section{IRON BROMID}

Dosage.-I-5 grains.

\section{IRON CARBONATE}

Preparations.

Saccharated Iron Carbonate.-Dose, 2-10 grains. Mass of Iron Carbonate (Vallet's Mass).-Dose, $\mathrm{I}-5$ grains.

Mixture of Iron Carbonate (Griffith's Mixture).Dose, I-4 drams.

IRON CHLORID

Synonyms.-Ferri Chloridum, Ferri Perchloridum, Ferric Chlorid.

Preparations.

Solution of Iron Chlorid.-Dose, 2-10 minims, well diluted.

Tincture of Iron Chlorid.-Dose, 5-60 minims.

Solution of Iron and Ammonium Acetate (Basham's Mixture).-Dose, I-4 drams.

IRON CITRATE

This is a scale preparation of iron and is soluble in water.

Dosage.-I-IO grains.

\section{IRON HYPOPHOSPHITE}

Has combined action of iron and phosphorus.

Dosage.-I-IO grains. 


\section{IRON IODID}

\section{Preparations.}

Saccharated Iron Iodid.-Dose, 5- 5 grains.

Syrup of Iron Iodid.-Dose, 5-30 minims.

\section{IRON OXID}

\section{Preparations.}

Hydrated Iron Oxid (Hydrated Iron).-Dose, I-4 drams.

Hydrated Iron Oxid with Magnesia (Arsenic antidote).-Prepared by mixing equal parts of the two following solutions : (I) Iron sulphate solution, 2 ounces ; water, $3 \frac{1}{2}$ ounces. (2) Magnesia, $2 \frac{1}{2}$ drams; water, 25 ounces. .

Saccharated Iron Oxid (Eisenzucker).-Dose, Io30 grains.

\section{IRON PHOSPHATE (SOLUBLE)}

Dosage.-I-5 grains.

\section{IRON SUBSULPHATE SOLUTION}

Synonym.-Monsel's Solution. Dosage.-2-10 minims.

\section{IRON SULPHATE}

Synonyms.-Green Vitriol, Copperas. Preparations.

Dried Iron Sulphate.-Dose, $\frac{1}{2}-2$ grains.

Blaud's Mass and Pills contain iron sulphate and potassium carbonate.

\section{IRON VALERIANATE}

Has combined action of valerian and iron. Dosage.-I-I 5 grains. 


\section{COMPOUND IRON PREPARATIONS}

Ammonium and Iron Citrate.-Dose, 3-Io grains. Ammonium and Iron Tartrate.-Dose, 5-Io grains. Iron and Potassium Tartrate.-Dose, 10-30 grains. Iron and Quinin Citrate.-Dose, 3-10 grains.

Iron and Strychnin Citrate.-Dose, 2-5 grains.

Iron, Quinin, and Strychnin Citrate-Dose, I-5 grains.

\section{Incompatibilities of Iron.}

Tannic acid and all substances containing it, as the vegetable infusions and alkalies, lime-water, carbonates, salicylates, mercuric salts, and albumin, should not be prescribed with iron.

Physiologic Action of Iron.

Astringent.-Applied to the mucous surface or the broken skin, iron salts act as powerful astringents, coagulating albumin and checking any secretions or bleeding.

Hematinic.-Taken internally iron causes an increase in the amount of hemoglobin in the blood, and also an increase of the red cells.

Intestinal Astringent.-In the intestines iron lessens the secretions and causes constipation. Unabsorbed iron combines with the sulphur in the bowels and forms iron sulphid, which colors the feces black.

Therapeutic Indications.

As an astringent to control bleeding points or to astringe relaxed tissues, as in pharyngitis, tonsillitis, and relaxed uvula. Also in diarrheas and hemorrhages of the gastro-intestinal tract.

As a hematinic in anemia of any form.

Administration.-As a local astringent the solution 
of iron chlorid or iron subsulphate are best. As a hematinic in anemia most any form may be employed, but the scale preparations (citrates and tartrates) are least constipating. Blaud's mass or the reduced iron are very useful in anemia.

In giving the tincture of iron chlorid use a glass tube to prevent action on the teeth.

The administration of an excessive amount of iron is indicated by frontal headache, constipation, and flushing of the skin.

\section{JALAP}

Derivation.-The tuberous roots of Jalapa Ipomea. Dosage.-5-20 grains.

Preparations.

Extract.-Dose, I-5 grains.

Compound Powder (Purging Powder) contains jalap and cream of tartar. Dose, 1 5-60 grains. Resin.-Dose, $\mathbf{I}-5$ grains.

Physiologic Action.

Purgative (Drastic). - Jalap stimulates the intestinal peristalsis and secretions and produces profuse watery bowel movements.

Depletant.-By its action on the bowels it lessens the circulation and engorgement in other parts of the body, and also aids in reducing fluid accumulations.

Therapeutic Indications.

As a purgatiz' for obstinate constipation and when rapid emptying of the bowels is necessary.

As a depletant in all forms of dropsy and anasarca. In cerebral congestion and plethora of any part of the body. 
JUNIPER ( $\left.{ }^{*}\right)$

KAMALA (*)

KINO (*)

KOLA $(*)$

KRAMERIA (*)

$\operatorname{LANUM}(*)$

\section{LEAD COMPOUNDS}

\section{LEAD ACETATE}

Synonyms.-Plumbi Acetas, Sugar of Lead.

Properties.-A white crystalline powder or mass with a sweet taste. It is soluble in water ( 2 parts), alcohol (25 parts).

Incompatibilities.-Acids, alkalies, tannin, carbonates, iodids, and lime-water.

Dosage. $-\frac{1}{2}-2$ grains.

Preparations.

Solution of Lead Subacetate (Goulard's Extract).The dilute form of lead-water is produced by taking 3 parts of this solution and 100 parts of water.

Cerate of Lead Subacetatc (Goulard's Cerate).

\section{LEAD CARBONATE}

Synonym.-White Lead.

Preparation.

Ointment. 


\section{LEAD IODID}

Dosage.-I-4 grains.

Preparation.

Ointment.

\section{LEAD OXID}

Synonym.-Litharge.

Preparation.

Plaster.-(Lead plaster, diachylon plaster, and lead oleate.)

Physiologic Action of Lead.

Astringent.-Applied externally lead salts produce coagulation of albumin, contraction of the vessels, and a decrease of secretions. Internally the salts act as astringents and cause constipation.

Therapeutic Indications.

Used extemally to dress wounds, ulcers, and eczema. Also as an astringent wash in leukorrhea, vulvitis, gonorrhea, and itching.

Internally some of the lead salts are employed in diarrhea, dysentery, intestinal hemorrhage, and nightsweats.

Toxicology.-Acute poisoning is characterized by nausea, vomiting, colic, constipation, cold and moist skin, and, finally, collapse.

Management.-Wash out the stomach, and give magnesium sulphate or sodium sulphate. Stimulate if necessary.

Chronic poisoning follows the prolonged use of lead as a medicine; the use of water contaminated by lead pipes; the use of hair dyes containing lead salts ; working in lead or using it much as a paint. The signs are: Anemia, severe abdominal colic centering around 
the umbilicus, obstinate constipation, headache, hard pulse, blue line on the gums near the margin of the teeth, and, later, various forms of paralyses, especially wrist-drop. To this set of symptoms the terms plumbism or saturnism are applied.

Management.-Stop the administration or source of the poison, then aid in its elimination by giving sulphates, as Epsom salt and also the iodids. For the colic, opium may be given, and for the paralysis, use electricity and strychnin.

\section{LEPTANDRA $(*)$}

\section{LITHIUM SALTS}

\section{LITHIUM BENZOATE}

Has the same action as Benzoic Acid (which see). Dosage. - 5-20 grains.

\section{LITHIUM BROMID}

Same action as, but less irritating than, Potassium Bromid.

Dosage. - 5-20 grains.

\section{LITHIUM CARBONATE}

Properties.-A white, odorless powder, soluble in water (80 parts) and insoluble in alcohol.

Dosage. - 5-1 5 grains.

\section{LITHIUM CITRATE}

Properties.-A colorless crystal, soluble in water (4 parts) and insoluble in alcohol.

Dosage. - 5-20 grains.

Physiologic Action. 
These salts taken internally render the urine alkaline; are said to prevent the formation of uric-acid calculi, and act as diuretics.

Therapeutic Indications.

They are chiefly used in gout, rheumatic affections, diseases of the bladder, and urinary calculi.

\section{LITHIUM IODID}

This salt acts the same as Potassium Iodid, but the former is less irritating.

Dosage. $-I-5$ grains.

\section{LITHIUM SALICYLATE}

Has same action as Sodium Salicylate (which see). Dosage.-10-30 grains.

LOBELIA $(*)$

\section{MAGNESIUM COMPOUNDS}

\section{MAGNESIUM CARBONATE}

Dosage. $-\frac{1}{2}-2$ drams.

Preparation.

Effervescent Magnesium Citrate Solution.-This is prepared by taking of magnesium carbonate $\mathrm{I} \frac{1}{2}$ drams and citric acid 3 drams, dissolved in 2 ounces of water. Stir these together until dissolved, then strain into a strong 8-ounce bottle and add enough syrup and water to nearly fill the bottle; then to the contents add $\frac{1}{2}$ dram of potassium bicarbonate, cork the bottle securely and quickly, and shake until the bicarbonate is dissolved. Dose, 2-8 ounces. 


\section{MAGNESIUM OXID}

Synonym.-Magnesia.

Dosage.-10-30 grains.

\section{MAGNESIUM SULPHATE}

Synonym.-Epsom Salt.

Dosage.-2-8 drams.

Physiologic Action.

Antacid.-Taken internally the carbonate and oxid act as antacids and gastric sedatives.

Cathartic.- The citrate and sulphate are saline purgatives, increasing the watery contents of the bowels; stimulating peristalsis, and causing watery bowel movements.

Therapeutic Indications.

As an antacid in gastric fermentation, acid dyspepsia, heartburn, and migraine.

As a cathartic to reduce fluid accumulations, as in dropsy (renal or cardiac), and for thoroughly evacuating the bowels.

Administration.-When Epsom salt is given for the purpose of reducing fluid accumulations it should be employed in concentrated solution.

\section{MANGANESE COMPOUNDS}

MANGANESE DIOXID

Synonyms.-Manganesc Pcroxid, Black Oxid of Manganesc.

Dosage.-I-IO grains.

\section{MANGANESE SULPHATE}

A colorless, transparent crystal, freely soluble in water and insoluble in alcohol.

Dosage.-I-IO grains. 


\section{POTASSIUM PERMANGANATE}

A dark purple crystal or powder, soluble in water (I6 parts).

It is incompatible with acids, alcohol, bromids, chlorids, tannin, and all forms of organic material.

Dosage. $-\frac{1}{2}-3$ grains.

Physiologic Action.

Antiseptic.-The permanganate is a strong antiseptic and oxidizing agent.

Hematinic.-Internally manganese increases the number of red cells and the hemoglobin of the blood.

Emmenagogue.-The dioxid and permanganate stimulate the menstrual flow.

Cholagogue.-The sulphate increases the flow of bile. Therapeutic Indications.

As an antiseptic potassium permanganate is employed in solution (I:50 to $\mathrm{I}: 1000$ ) in mouth affections, rhinitis, diphtheria, gonorrhea, leukorrhea, and also as an application to wounds. Owing to its oxidizing powers this salt is used as a deodorizer on putrid wounds and ulcers.

As a hematinic in anemia of the chlorotic type.

As an emmenagogue in suppressed menstruation due to anemia or atony.

\section{MENTHA PIPERITA}

Derivation.-The leaves and tops of Mcutha Piperita.

Synonym.-Peppermint.

Constituents.-Menthol and a volatile oil.

Preparations.

Oil.-Dose, I-5 minims. 
Spirit.-Dose, 5-1 5 minims.

Water.-Dose, I-8 drams.

Menthol.- A stearopten from the oil of peppermint. Soluble freely in alcohol and in 4 parts of olive oil. Dose, $\mathrm{I}-5$ grains.

Physiologic Action.

Local Anodyne.-Applied to the skin or mucous membrane the oil or menthol produce first a cooling sensation, followed by numbness.

Carminative.-Peppermint aids in the expulsion of flatus.

Therapeutic Indications.

As an analgesic in toothache, headache, neuralgia, gastralgia, and itching.

As a carminative in flatulent dyspepsia, infantile colic, and diarrhea.

\section{MERCURY COMPOUNDS}

\section{AMMONIATED MERCURY}

Synonym.-White Precipitate.

Preparation.

Ointment.

\section{MERCURY BICHLORID}

Synonyms.-Hydrargyri Chloridum Corrosivum, Corrosive Sublimate.

Properties.-A white crystalline mass, soluble in water (20 parts), in alcohol ( 5 parts).

Dosage. $-\frac{1}{60}-\frac{1}{12}$ grain.

Preparation.

Yellow Wash (Lotio Flava).-Prepared by mixing $\frac{1}{2}$ dram of mercury bichlorid with I pint of limewater. 


\section{MERCURY CHLORID}

Synonyms.-Hydrargyri Chloridum Mite, Calomel, Mild Chlorid of Mercury.

Properties.-A white powder devoid of odor and taste. Insoluble in water or alcohol.

Dosage. $-\frac{1}{10}$ IO grains.

Preparation.

Black Wash (Lotio Nigra).-Prepared by adding I dram of calomel to I pint of lime-water.

MERCURY IODID (RED)

Synonym.-Mercury Biniodid.

Properties.-A red powder, insoluble in water, but soluble in a solution of potassium iodid.

Dosage. $-\frac{1}{16}-\frac{1}{8}$ grain.

Preparation.

Solution of Arsenic and Mercuric Iodid (Donovan's Solution).-Dose, I-IO minims.

\section{MERCURY IODID (YELLOW)}

Synonyms.-Protoiodid of Mercury, Green Iodid of Mercury.

Properties.-A yellow or green powder, devoid of taste and odor, and insoluble in water or alcohol.

Dosage. $-\frac{1}{4}$ - I grain.

$$
\text { MERCURY OXID (RED) }
$$

Synonym.-Red Precipitate.

Preparation.

Ointment.

$$
\text { MERCURY OXID (YELLOW) }
$$

Preparations.

Ointment.

Oleate of Mercury. 
MERCURY SUBSULPHATE (YELLOW)

Synonym.-Turpeth Mineral.

Dosage.-2-4 grains.

METALLIC MERCURY PREPARATIONS

Mercury with Chalk (Hydrargyrum cum Creta, Gray Powder) contains 38 per cent. of mercury. Dose, $\frac{1}{2}-10$ grains.

Mercury Mass (Massa Hydrargyri, Blue Mass) contains 33 per cent. of mercury. Dose, $\frac{1}{2}$ I 5 grains.

Mercury Ointment (Blue Ointment) contains 50 per cent. of mercury.

Mercury Plaster contains 30 per cent. of mercury.

Mercury Plaster (with Ammoniac) contains is per cent. of mercury.

Incompatibilities of Mercury Compounds.

Alkalies, alkaloids, albumin, bromids, lime-water, tannin, and infusions.

Physiologic Action.

Antiseptic.-The bichlorid is one of the best and most efficient of the antiseptics. A I : rooo solution destroys bacterial life. It is extensively used in surgery for all antiseptic purposes except the sterilization of metallic instruments, which the chemic action will corrode.

Parasiticide.-Mercury preparations rapidly destroy animal and vegetable parasites that may be found on the skin.

Caustic.-Some of the mercurials (red iodid) act upon the skin as an irritant and, if the action is prolonged, as a caustic.

Cathartic.-All mercury preparations are irritating to the intestinal tract, producing looseness of the 
bowels, but calomel, gray powder, and blue mass are most used for this purpose.

Cholagogue.-Calomel is said to indirectly increase the flow of bile.

Hematinic.-Mercury in small doses increases the number of red cells in the blood, but its prolonged action will produce anemia.

Diuretic.-Calomel when combined with diuretics augments their action.

Sialagogue.-Mercury is excreted in the saliva, which secretion it greatly increases.

Antisyphilitic.-Mercurials cause the absorption and disappearance of syphilitic processes.

Therapeutic Indications.

As an antiseptic for sterilizing the hands; the site of operation; the basins, etc. As a wet dressing for infected wounds and as an irrigating solution.

As a parasiticide the bichlorid, blue mass, and ammoniated mercury or the oleate are used for pediculosis, favus, and ringworm.

As a cathartic in the beginning of the acute fevers, especially in the form of calomel, blue mass, or gray powder. It is best to follow the mercurial with a saline.

As a hematinic in anemia.

As a diuretic in nephritis, especially if given with another diuretic, as digitalis and squill.

As an antisyphilitic, mercury may be employed in any stage of the disease.

Administration.-Calomel is given as a cathartic in several ways, among the most frequent are: $\frac{1}{10}$ grain every half hour until ten doses are taken, or I grain 
every hour until five doses are taken, or in mass doses of 5 or 10 grains. In cases where there is vomiting and all drugs are ejected as soon as taken, a most excellent plan is to place 5 grains of calomel powder on the patient's tongue and give a small swallow of water. If vomiting occurs, most of the calomel will be retained, as it adheres to the stomach wall. It is always best to follow the administration of calomel with one of the saline purgatives, for two reasons : Calomel acts mostly on the upper part of the intestines, while the salts will effect the lower portion, and, if a large dose of calomel has been given, the saline will wash out the excess before poisoning occurs. Sodium bicarbonate should be combined with mass doses of calomel, as this prevents part of the calomel from being converted into mercury bichlorid.

In syphilis, mercurials may be administered in many ways. By mouth, the best preparation to be given is probably the yellow or protoiodid. The bichlorid, the red iodid, and gray powder are also much used. By hypodermic, the bichlorid is employed in doses of $\frac{1}{12}$ grain in 15 minims of water, given every other day, or gray oil may be used by this method. By inunction, the blue ointment or the oleate are most used: I dram is well rubbed into the skin every few days, selecting a different site for each application. By fumigation, calomel is generally employed: I 5 grains of calomel are placed in a metal or porcelain dish over an alcohol lamp, which is placed under the chair of the patient, and about the entire body of the patient excepting the head is placed a blanket in the shape of a tent. By baths, 2 drams of the bichlorid are dissolved in 20 gal- 
lons of water and to this 20 drops of hydrochloric acid are added to aid in the solution of the mercury salt. The patient remains in the bath for only a few minutes. As a local application to the primary lesions, the black or yellow wash are excellent.

Pretoxic Signs.-Slight salivation, vague abdominal colic, and looseness of the bowels.

Toxicology.-Acute Poisoning.-Great flow of saliva, vomiting (which may become bloody), diarrhea, suppression of urine, cold, moist skin, weak pulse, and collapse.

Management.-Empty and wash the stomach. Give milk, white of egg, flour paste, and starch-water. Stimulate with ammonia, brandy, and strychnin.

Chronic Poisoning, or Mercurialism.-This is characterized by anemia, emaciation, dyspepsia, trembling, dizziness, headache, fetor of the breath, soreness of the gums, looseness of the teeth, and various forms of paralyses.

\section{METHYLENE-BLUE (*)}

MUSK $(*)$

\section{MUSTARD (*)}

NAPHTOL $(*)$

\section{NITRIC ACID}

Definition.-A clear, fuming liquid, which contains 68 per cent of absolute nitric acid in water.

Dosage.-I-5 minims, well diluted.

Preparations.

Dilute Nitric Acid (Io per cent. strength).-Dose, 5-20 minims. 
Nitrohydrochloric Acid (Aqua Regia, Nitromuriatic Acid).-Dose, I-5 minims.

Physiologic Action.

Caustic.-Applied externally it produces a burning sensation, followed by destruction of tissue.

Cholagogue.-This acid increases the flow of bile and stimulates the liver. This is particularly true of the nitrohydrochloric acid.

Therapeutic Indications.

As a caustic nitric acid is much used for cauterizing the bites of animals, and in the treatment of chancroids, warts, indolent ulcers, and condylomata.

Internally these acids are useful in jaundice, chronic hepatitis, and diarrhea.

Toxicology.-See under Hydrochloric Acid.

\section{NITROGLYCERIN}

Derivation.-A yellow, clear, oily, explosive liquid, formed by the action of nitric and sulphuric acids on glycerin. It is soluble in alcohol and only slightly in water.

Synonyms.-Glonoin, Trinitrin, Glyceryl Trinitrate. Preparation.

Spirit (Spirit of Glonoin, Solution of Trinitrin, Spiritus Glycerylis Nitratis).-This is a I per cent. solution of nitroglycerin in alcohol. Dose, I-3 minims.

Action and Use.

The action and use of nitroglycerin is the same as that of amyl nitrite, except that the action of the former is slower and more prolonged. 


\section{NUX VOMICA}

Derivation.-The seeds of Strychnos Nux Vomica. Synonyms.-Poison Nut, Quaker Button, Dog Button.

Constituents.-Alkaloids : Strychnin and Brucin.

Dosage.-I-4 grains.

Preparations.

Extract.-Dose, $\frac{1}{8}-\frac{1}{2}$ grain.

Fluidextract.-Dose, I-4 minims.

Tincture.-Dose, 5-I 5 minims.

Strychnin.-Dose, $\frac{1}{100-\frac{1}{30}}$ grain.

Strychnin Nitrate.-Dose, $\frac{1}{100}-\frac{1}{30}$ grain.

Strychnin Sulphate.-Dose, $\frac{1}{100-\frac{1}{30}}$ grain.

Physiologic Action.

Stomachic.-Nux vomica excites the secretion of the gastric juice, sharpens the appetite, and stimulates the motor power of the stomach and intestines.

Cardiac Stimulant.-Strychnin is one of our best cardiac stimulants; it increases both the rate and power of the heart.

Respiratory Stimulant.-The number and depth of the respirations are increased by this drug.

Spinal Stimulant.-Strychnin exerts its energy on the motor fibers of the cord, producing increased muscle tone and reflexes. In very large doses it causes muscular twitchings and convulsions.

Cerebral Stimulant.-This drug stimulates the special centers of the brain, producing acuteness of hearing, sight, and smell.

Therapeutic Indications.

As a stomachic in atonic dyspepsia, anorexia, anemia, and constipation. 
As a stimulant in shock, syncope, cardiac failure of the acute fevers, and in drug poisoning.

As a muscle tonic in paralysis of apoplexy, neuritis, arsenic- and mercury-poisoning.

Administration.-As a stomachic, the best preparation is the tincture. As a stimulant, any of the salts of strychnin are to be preferred. It is well to remember that the continuous use of strychnin in the acute fevers, especially typhoid fever, may prevent the temperature from becoming normal as soon as it would otherwise.

Pretoxic Signs.-Slight twitching of the muscles, increase of reflex action, and restlessness.

Toxicology.-The symptoms of strychnin-posioning are increased reflex excitability, muscular twitching, rigidity of the neck, rapid pulse, acuteness of hearing, and convulsions of the tetanic type with opisthotonos.

Management.-Give rectal injections of chloral and bromids. Wash out the stomach and administer tannic acid. Inhalations of amyl nitrite are useful.

\section{OPIUM}

Derivation.-The inspissated juice of Papaver Somniferum.

Constituents.-Morphin, codein, narcein, narcotin, papaverin, and other alkaloids of less importance.

Dosage. $\frac{1}{4} 2$ grains.

Preparations.

Powedered Opium.-Dose, $\frac{1}{4}-3$ grains.

Extract.-Dose, $\frac{1}{8}$ - I grain.

Tincture (Laudanum).-Dose, 3-15 minims.

Deodorized Tincture.-Dose, 3-15 minims. 
Camphorated Tincture (Paregoric).-Dose, I-4 drams.

Vinegar (Black Drop).-Dose, 3-15 minims.

Wine (Sydenham's Laudanum).-Dose, 3-15 minims.

Deodorized Opium.-Dose, $\frac{1}{4}$ I grain.

Powder of Opizum and Ipecac (Dover's Powder).-

Dose, 3-I 5 grains.

Plaster.

Morphin.-Dose, $\frac{1}{8}-\frac{1}{4}$ grain.

Morphin Hydrochlorate.-Dose, $\frac{1}{8}-\frac{1}{4}$ grain.

Morphin Sulphate.-Dose, $\frac{1}{8}-\frac{1}{4}$ grain.

Codein.-Dose, $\frac{1}{4}-2$ grains.

Codein Phosphatc.-Dose, $\frac{1}{4}$ I grain.

Codein Sulphate.-Dose, $\frac{1}{4}-2$ grains.

Heroin is an artificial alkaloid formed by the action of acetic acid on morphin.-Dose, $\frac{1}{24}-\frac{1}{6}$ grain.

Heroin Hydrochlorate.-Dose, $\frac{1}{24-\frac{1}{6}}$ grain.

Dionin (an artificial alkaloid).-Dose, $\frac{1}{4}-\mathrm{I}$ grain.

Cotarnin Hydrochlorate (an oxidation product of

Narcotin, known as Stypticin, see Part IV.).-

Dose, $\frac{1}{2}-4$ grains.

\section{Physiologic Action.}

Hypnotic.-Opium is our greatest sleep producer. It first stimulates the brain, slightly increasing the intellection and imagination, and then quiets the nervous system, producing sleep.

Analgesic and Antispasmodic.-Opium depresses the motor and sensory fibers, thus producing a decrease of motor excitability and a blunting of the pain sense.

Cardiac Stimulant.-Opium in medicinal doses slows the heart's action, but increases the force. 
Myotic.-Given internally in moderate doses this drug produces contraction of the pupils.

Secretory Depressor.-Opium lessens all the secretions of the body except the perspiration, which is increased. Opium causes constipation.

Therapeutic Indications.

As an hypnotic in cases of sleeplessness accompanied by pain, and in insomnia of exhausting fevers, also in the wakefulness of chronic alcoholism, mania, and insanity.

As an analgesic in pain from nearly all causes except congestion of the brain. Opium should not be administered if its action will dispel any points in making a diagnosis.

As an antispasmodic in epilepsy, asthma, intestinal colic, convulsions, tetanus, cough, biliary, renal, and vesical colic.

As a secretory depressor in diarrhea, dysentery, cholera, ptyalism, and diabetes.

Comparative Action.-Morphin possesses the same general action of opium, except that the former is less narcotic, more analgesic, more rapid, less constipating, less diaphoretic, and more depressing to the heart.

Codein has the same action as morphin, except that the former is more feeble and has especial powers as a cough sedative.

Heroin has the same cough-sedative action as codein, but is more depressing.

Dionin is similar to heroin.

Cotarnin is especially active as a vasoconstrictor.

Administration.-As an analgesic morphin is preferable. As a secretory depressor, powdered opium, 
laudanum, and paregoric are to be employed. As a cough sedative codein or heroin should be chosen. Children bear opium and its derivatives very poorly, and it should be given to them in very small doses and with extreme caution. In susceptible persons and some alcoholics the narcotic should be reinforced by some stimulant, as atropin or strychnin.

Pretoxic Signs.-Tendency to pupillary contraction, drowsiness, dryness of the mouth, sweating.

Toxicology.-Acute Poisoning.-Small pupils, slow, full pulse, becoming rapid, slow respirations, hot skin, cyanosis, and asphyxia.

Management.-Wash out the stomach at once or give emetics followed by tannic acid or potassium permanganate. Keep the patient aroused by constant flagellation. Give atropin, coffee enemata, ammonia, strychnin, but not digitalis.

Chronic Poisoning.-This is characterized by smallness of the pupils, loss of appetite, anemia, insomnia, mental unrest, constipation, muscular weakness, tremor, immorality. The patient will steal or tell untruths in order to procure the drug.

Management.-Withdraw the drug and substitute hyoscin or cannabis indica temporarily. Overcome insomnia by hydrotherapeutic methods and the bromids, chloral, or paraldehyd. Give tonics and good food and, above all, use moral suasion.

\section{OX-GALL}

Derivation.-The fresh bile or gall of Bos Tauris, the domestic ox.

Synonyms.-Fel Bovis, Fel Tauri. 
Dosage.-5-I 5 grains.

Physiologic Action.

In the stomach it interferes with digestion, and for this reason it is much employed in keratin-covered tablets, which pass through the stomach unaffected. Having gained entrance to the intestines it increases the flow of bile and stimulates peristalsis. For this last purpose it is given in the form of an enema by adding 15 grains of powdered ox-gall to the regular enema.

\section{PANCREATIN}

Derivation.-A mixture of ferments from the fresh pancreas of the hog.

Dosage.-5-I 5 grains.

Action and Use.

Pancreatin has the power of digesting meats, starches, sugars, and of emulsifying fats. It can act only in an alkaline solution, therefore it does not act in the stomach unless sodium bicarbonate be given with it to neutralize the acid of the stomach. Some authorities say that a minute amount of acid will destroy pancreatin, hence its value as a digestive agent is questionable. It is much used, however, as an ingredient of "peptonizing" powders for preparing partially digested milk, junket, etc.

\section{PAPAIN}

Derivation.-A ferment derived from the juice of Carica Papaya.

Synonyms.-Papayotin, Papoid, Papaw

Dosage.-I -5 grains. 


\section{Action and Use.}

This ferment is capable of digesting proteid, carbohydrates, and fats in a neutral, alkaline, or acid medium. Whether it will do all this is much doubted.

Locally it will dissolve necrotic tissue, and is used for this purpose in diphtheria, warts, and small growths.

\section{PARALDEHYD $\left({ }^{*}\right)$}

\section{PEPSIN}

Derivation.-A ferment derived from the stomach of the pig.

\section{Preparations.}

Pure Pepsin.-Dose, 5-I 5 grains.

Saccharated Pepsin.-Dose, 5-6o grains.

Compound Powder (Lactated Pepsin).-Dose, 5I 5 grains.

Action and Use.

Pepsin has the power of digesting proteid matter in an acid solution. According to the U. S. Pharmacopeia, I grain of pepsin must be capable of digesting at least 3000 grains of proteid matter.

\section{PHENACETIN}

Derivation.-A white, odorless, and tasteless powder, produced by the action of glacial acetic acid on a coal-tar derivative. It is insoluble in water.

Dosage.-I-IO grains.

Physiologic Action.

Antipyretic.-Phenacetin will cause a fall of febrile temperature. 
Analgesic. - The most important action of this drug is its power to relieve pain.

Comparative Action.-Compared to acetanilid and antipyrin, phenacetin is much safer and less depressing, but it is slightly less powerful.

Therapeutic Action.

As an antipyretic in scarlatina, rheumatism, and influenza.

As an analgesic in neuralgias, influenza, headaches, rheumatism, ovaralgia, and locomotor ataxia.

Toxicology.-See Acetanilid. The writer has never seen reported a case of fatal poisoning by this drug.

\section{PHOSPHORUS COMPOUNDS}

Preparations of Phosphorus.

Oil of Phosphorus.-Dose, I-4 minims.

Spirit.-Dose, 8-30 minims.

Elixir.-Dose, $\frac{1}{2}-\mathrm{I}$ dram.

Physiologic Action.

Bone Stimulant.-Given internally phosphorus stimulates the growth of bone.

Hematinic.-This drug aids in the formation of blood, causing an increase of the red cells.

Nervine.-Phosphorus is said to stimulate nerve reconstruction and act as a tonic to the nervous system.

Therapeutic Indications.

As a bone stimulant in rachitis, osteomalacia, and caries of bone.

As a nerve tonic in nervous and sexual exhaustion, typhoid fever, melancholia, and mania.

As a hematinic in anemia, leukemia, and pseudoleukemia. 
Toxicology.-Acute Poisoning.-Nausea, vomiting, abdominal colic, jaundice, scanty, high-colored urine, and collapse.

Management.--Wash out the stomach. Give oil of turpentine (especially old oil) and copper sulphate as antidotes. Stimulate.

Chronic poisoning occurs in persons employed where phosphorus is used and is characterized by anemia, bronchitis, spongy gums, and necrosis of bone, especially of the lower jaw.

\section{DILUTE PHOSPHORIC ACID}

This is a ro per cent. solution, and is used in those diseases where phosphorus is indicated.

Dosage.-10-60 minims.

\section{GLYCERINOPHOSPHATES}

Calcium Glyccrinophosphate.-Dose, 2-5 grains. Iron Glycerinophosphate.-Dose, 2-5 grains. Magnesium Glycerinophosphate.-Dose, I-5 grains. Potassium Glycerinophosphate.-Dose, 2-10 minims. Sodium Glycerinophosphate.-Dose, 2-10 grains.

These preparations are generally administered in liquid form in nervous disorders, as neurasthenia, deficient nerve nutrition, and anemia.

\section{HYPOPHOSPHITES}

Ammonium Hypophosphite.-Dose, I-3 grains. Calcium Hypophosphite.-Dose, 5-20 grains. Iron Hypophosphite.-Dose, 3-Io grains. Mangancse Hypophosphitc.-Dose, 3-10 grains. Potassium Hypophosphite.-Dose, 5-20 grains. 
Quinin Hypophosphite.-Dose, I-5 grains.

Sodium Hypophosphite.-Dose, 5-20 grains.

Action and Use.

The hypophosphites are used mostly in tuberculosis, nervous exhaustion, anemia, and depraved conditions of the system.

The ammonium salt is especially useful in bronchitis.

These salts are generally administered in the form of a syrup.

\section{PHOSPHATES}

Calcium Phosphate.-Used in rickets and anemia. Dose, 5-20 grains.

Iron Phosphate.-Used in anemia and amenorrhea. Dose, I-5 grains.

Sodium Phosphate.-This salt is very useful, and is much employed in jaundice, hepatitis, biliousness, rickets, and headaches due to gastric disorder.

\section{ZINC PHOSPHID}

Used as a substitute for phoshorus.-Dose, $\frac{1}{20}-\frac{1}{8}$ grain.

\section{PHYSOSTIGMA}

Derivation.-The seed or bean of Physostigma Venenosum.

Synonym.-Calabar Bean.

Constituents.-Physostigmin or Eserin.

Dosage. $-\frac{1}{12-\frac{1}{2}}$ grain.

Preparations.

Extract.-Dose, $\frac{1}{12}-\frac{1}{2}$ grain.

Tincture.-Dose, 5-20 minims. 
Physostigmin (or Eserin) Salicylate.-Dose, $\frac{1}{120}$ $\frac{1}{40}$ grain.

\section{Physiologic Action.}

Spinal Depressant.-Eserin depresses both sides of the cord, especially the sensory tracts, and lessens sensation and reflex action.

Involuntary Muscle Stimulant.-This drug stimulates the involuntary muscle of the gastro-intestinal tract, thus increasing peristalsis, and of the vascular coats, causing vasoconstriction.

Myotic.-Eserin produces pupillary contraction.

Therapeutic Indications.

As a spinal depressant in tetanus, strychnin-poisoning, convulsions, and asthma.

As a muscle stimulant in gastric atony and dilatation, in constipation, and in post-operative paresis of the bowels with distention.

As a myotic in glaucoma and ciliary paresis.

Toxicology.-Muscular weakness, tremor, relaxation, small pupils, slow pulse and respirations.

Management.-Give atropin, strychnin, coffee, and ammonia.

\section{PHYTOLACCA $\left.{ }^{*}\right)$}

\section{PICRIC ACID}

Derivation.-A yellow crystal with a very bitter taste, formed by the action of nitric acid on creosote.

Synonyms.-Picronitric Acid, Carbazotic Acid, Trinitrophenol.

Action and Use.

Picric acid acts somewhat like quinin as an antiperiodic, and is used in the treatment of malaria. 
Externally it is used extensively as a dressing for burns. The writer is told that in some of the iron mines and works of Pennsylvania it is exclusively employed for this purpose. It is applied as a saturated watery solution.

It is also recommended in the treatment of trichinosis.

\section{PICROTOXIN $(*)$}

\section{PILOCARPUS}

Derivation.-The leaves of Pilocarpus Jaborandi.

Synonym.-Jaborandi.

Constituent.-Pilocarpin, a liquid alkaloid.

Dosage. - 5-30 grains.

Preparations.

Fluidextract.-Dose, 5-30 minims.

Pilocarpin Hydrochlorate.-Dose, $\frac{1}{12}-\frac{1}{4}$ grain.

Physiologic Action.

Sialagogue. - Pilocarpin greatly increases the secretion of saliva.

Diaphoretic.-This drug is our most powerful sweat producer. Its action may continue for from one to three hours, producing from twelve to twenty-four ounces of perspiration.

Galactagogue.-The secretion of milk in nursing mothers is greatly increased by the use of this drug.

Cardiac Depressant.-Pilocarpin, being unique in its other actions, is less frequently used because of its depressing effect on the heart.

Myotic.-The pupils are contracted by the use of this drug. 
Therapeutic Indications.

As a diaphoretic in fluid accumulations, as dropsy. In uremia, chronic nephritis, and eclampsia.

As a myotic in glaucoma.

The use of this drug has been recommended in baldness as a local applicant.

Pretoxic Signs.-Disturbance of vision and vomiting.

Toxicology.-Poisoning is characterized by vomiting, purging, salivation, parotid tenderness, contracted pupils, vesical tenesmus, yawning, slow pulse, which becomes rapid, and collapse.

Management.-Empty the stomach by a tube or emetics. Give tannic acid. Stimulate with atropin and ammonia.

\section{PODOPHYLLUM}

Derivation.-The rhizome and roots of Podophyllum Peltatum.

Synonyms.-Mandrake, May Apple.

Dosage.-5-30 grains.

Preparations.

Extract.-Dose, 2-Io grains.

Fluidextract.-Dose, 5-30 minims.

Podophyllin (Resin).-Dose, $\frac{1}{8}-\frac{1}{2}$ grain.

Physiologic Action.

Cholagogue Cathartic.-Internally mandrake acts as a gastro-intestinal stimulant and increases the flow of bile, producing by this combined action free bowel movements containing much biliary material.

Therapeutic Indications.

Mandrake is employed in biliousness and liver disorders, and in infantile diarrhea when the stools lack color. 
Mandrake is commonly known as the vegetable calomel.

\section{POMEGRANATE}

Derivation.-The root of Punica Granatum.

Synonym.-Granatum.

Dosage.-I-2 drams.

Preparation.

Pelletierin Tannate (Alkaloid).-Dose, I-I 2 grains. Action and Use.

The chief use of pomegranate is as an anthelmintic for the tapeworm. This drug is also employed as an intestinal astringent in diarrhea.

\section{POTASSIUM COMPOUNDS}

\section{POT ASSIUM ACETATE}

A white, deliquescent, crystalline powder, soluble in water and alcohol. Dose, 5-60 grains.

Potassium acetate is used as a diuretic in acute nephritis and the contagious fevers. It is also useful in rheumatism, gout, scurvy, eczema, and lithiasis.

\section{POTASSIUM ARSENITE \\ (See Arsenic Compounds) \\ POTASSIUM BICARBONATE}

A white crystal, soluble in water, but insoluble in alcohol. It is used for the same purposes as the acetate. Dose, 10-60 grains.

\section{POTASSIUM BICHROMATE}

An orange-colored crystal, soluble in water and alcohol. 
Applied to the skin this salt acts as an irritant and caustic. It is used as such in the treatment of warts, condylomata, and vegetations.

Internally it acts as an expectorant, and is employed in bronchitis and aphonia.

Dosage. $\frac{1}{16-\frac{1}{6}}$ grain.

\section{POTASSIUM BITARTRATE}

Synonyms.-Cream of Tartar, Acid Potassium Tartrate, Tartar Crystals.

This salt given in moderate doses acts as a saline cathartic without griping. It is also a diuretic and renders the urine alkaline.

Dosage.-20 grains to I ounce.

\section{POTASSIUM BROMID}

Incompatibilities.-Acids, alkaloids, and metallic salts.

Dosage. - 5-60 grains.

Physiologic Action.-(This answers for all bromids.)

Gastric Irritant.-Potassium bromid is irritating to the stomach. It lessens the motor power, causes flatulence, and may produce dyspepsia.

Nerve Sedative.-The bromids quiet the nervous system, producing tranquillity, calmness, and decrease of excitability.

Hypnotic.-By continued action on the brain centers the bromids produce a more or less natural sleep.

Antispasmodic.-These salts depress the motor centers, lessening the reflexes and motor excitability.

Cardiac Sedative.-In small doses the heart is not 
affected, but in larger amounts the force and rate of the heart are decreased.

\section{Therapeutic Indications.}

As a sedative in nervous excitability, hysteria, seasickness, vomiting, and the nervous symptoms of the menopause.

As an hypnotic in nervous insomnia.

As an antispasmodic in delirium tremens, epilepsy, whooping-cough, tetanus, asthma, dysmenorrhea, and strychnin-poisoning.

Administration.-Bromids should never be given in dry form, but always in solution and well diluted, after meals, and not on an empty stomach.

Comparative Action.-Potassium bromid is more depressing to the heart and more irritating to the stomach than other bromids. Sodium bromid is more hypnotic and less irritating than the former. Ammonium bromid is not depressing to the heart, but is very irritating to the stomach. Lithium bromid is said to be the most hypnotic and least irritating. Strontium bromid is non-irritating and is an excellent hypnotic.

Pretoxic Signs.-Acneal rash, dulness of intellect, and drowsiness.

Toxicology.-By the prolonged use of the bromids a certain set of symptoms appear, to which the name bromism is applied. Fetid odor to the breath, apathy, dull expression, dyspepsia, decrease of skin sensibility, hallucinations, and delirium.

\section{POTASSIUM CHLORATE}

This salt is much employed as a gargle and mouthwash in stomatitis, pharyngitis, tonsillitis, and scarlet 
fever. One objection to its use in scarlet fever is its irritating action on the kidneys and its liability to cause nephritis. It is used as a 4 per cent. solution.

\section{POTASSIUM CITRATE}

Has the same action as the Acetate.

Dosage. $-5-30$ grains.

\section{POT ASSIUM IODID}

Incompatibilities.-Starch, sweet spirit of niter, alkaloids, metallic salts, and mercury protoiodid.

Dosage. - 5-60 grains.

Physiologic Action.-(This answers for all iodids.)

Gastric Irritant.-The iodids are very irritating to the stomach and may produce nausea, vomiting, and signs of indigestion.

Absorbent.-The iodids have a peculiar action in causing the absorption of connective tissue, especially that which is newly formed. They also aid in reducing fluid accumulations.

Antisyphilitic.-This is probably the most important action of the iodids, and they are extensively employed in the treatment of syphilis.

Therapeutic Indications.

As an absorbent in chronic inflammations, as pleurisy, bronchitis, adenitis, arteritis, aneurysm, asthma, emphysema, and chronic rheumatism.

As an antisyphilitic in the late secondary and the tertiary manifestations.

Administration.-The iodids should never be given in dry form, but always in well-diluted solution.

In syphilis the dose must be steadily increased. To 
facilitate this the drug is dissolved in water, so that I minim of the solution is equal to $\frac{1}{2}$ grain of the iodid.

The iodids are useful in the treatment of poisoning by arsenic and lead.

Toxicology.-Prolonged use of the iodids produce a series of symptoms to which the term iodism is applied. They are nausea, vomiting, and signs of dyspepsia, running and watering of the eyes, nose, and mouth. The writer has known three doses of 5 grains each to have produced in an adult profuse watering of the eyes and nose. Skin eruptions are common, also are anemia, loss of flesh, wasting of the breasts, and melancholia.

\section{POTASSIUM NITRATE}

Synonyms.-Saltpeter, Niter.

Dosage.-3-5 grains.

This salt produces gastro-intestinal irritation and cardiac depression. It is a slight diuretic and an antispasmodic. For this last action it is used in the treatment of asthma by inhaling the fumes from burning paper, which has been immersed in a 20 per cent. watery solution of saltpeter and then dried.

\section{POTASSIUM PERMANGANATE \\ (See under Manganese Compounds) \\ POTASSIUM AND SODIUM TARTRATE}

Synonym.-Rochelle Salt.

Dosage. - Up to I ounce.

The action is the same as of Potassium Bitartrate. 
Preparation.

Compound Effervescing Powder (Seidlitz Powder). -This contains: (a) 40 grains of sodium bicarbonate and 2 drams of Rochelle salt; $(b) 35$ grains of tartaric acid. Dissolve each powder(a) and (b)-in water, mix, and drink while effervescing.

PRUNUS VIRGINIANA (*)

QUASSIA (*)

QUININ SALTS

(See under Cinchona)

\section{RESORCIN}

Properties.-A coal-tar product of the carbolic acid class, occurring in fine pinkish crystals, soluble in water (freely) and alcohol.

Dosage.-I-3 grains.

Action and Use.

Antiseptic.-Resorcin is a strong antiseptic, and is used as such in cystitis (as an irrigation), gastric fermentation, cholera, diarrhea, and parasitic skin diseases.

Antipyretic.-Resorcin will reduce a febrile temperature, but is too dangerous to be used as such.

Resorcin has a special action in keratotic and scaly diseases of the skin, in which it very quickly removes the horny scales. It is used in eczema, psoriasis, dandruff, and other scaly affections.

\section{RHUBARB}

Derivation.-The root of Rheum Officinale.

Synonym.-Rheum.

Dosage.-3-20 grains. 


\section{Preparations.}

Extract.-Dose, I-5 grains.

Fluidextract.-Dose, 3-20 minims.

Tincture.-Dose, I-4 drams.

Aromatic Tincture.-Dose, $\frac{1}{2}-2$ drams.

Sweet Tincture.-Dose, I-4 drams.

Mixture of Rhubarb and Soda.-Dose, I-8 drams. Syrup.-Dose, I-4 drams.

Aromatic Syrup.-Dose, $\frac{1}{2}-2$ drams.

Compound Powder (Gregory's Powder).-Dose, I 5-60 grains.

Physiologic Action.

Stomachic.-Taken internally, rhubarb stimulates both the motor and secretory powers of the stomach and sharpens the appetite.

Cathartic.-In the intestines it stimulates peristalsis, causing free bowel movements, usually attended with griping.

Astringent.-Owing to the large amount of tannic acid, rhubarb decreases the intestinal secretions and constipation follows the cathartic action.

Therapeutic Indications.

As a stomachic in atonic dyspepsia, flatulence, anorexia, and during convalescence.

As a cathartic, when it is desirable to empty the bowels and then have them rest, as in diarrhea of children, fermentative diarrhea, and biliousness.

Administration.-Rhubarb is seldom given alone on account of its griping, but is combined with other laxatives and correctives.

Rhubarb may cause the urine to become dark yellow or red in color. 


\section{SACCHARIN $(*)$}

\section{SALICYLIC ACID COMPOUNDS}

\section{SALICYLIC ACID}

Derivation.-A constituent of such plants as wintergreen and sweet birch, from which it is obtained. It is also extensively prepared from carbolic acid.

Incompatibilities.-Spirit of nitrous ether, iron salts, and potassium iodid.

Properties.-A white, fluffy, crystalline powder, soluble in water (500 parts), alcohol (3 parts), olive oil ( 25 parts). It is odorless and has a sweet taste.

Dosage. $-5-30$ grains.

\section{SALICYLATES}

Cinchonidin Salicylate.-Dose, I-IO grains.

Lithium Salicylate.-Dose, I-I o grains.

Methyl Salicylate.-Artificial oil of wintergreen.

Sodium Salicylate.-Dose, 5-30 grains.

\section{SALOL}

A product of the action of carbolic acid on salicylic acid. It is insoluble in water. Dose, I-Io grains.

\section{SALICIN}

A glucosid derived from willow bark. It is a white, bitter, crystalline powder, soluble in water. Dose, 530 grains.

\section{SALOPHEN}

A white, scaly powder, without odor or taste, and insoluble in water. Dose, 5-15 grains. 


\section{ASPIRIN}

This preparation is decomposed by water or sodium bicarbonate, and should be given in dry form. Dose, 5-15 grains.

Physiologic Action of Salicylic Acid Compounds.

Antiseptic.-Salicylic acid externally and the salicylates internally act as antiseptics.

Anhidrotic.-Applied to the skin, salicylic acid hardens it and prevents excessive sweating.

Cardiac Depressant.-Internally these compounds decrease the cardiac force, and in large doses are distinctly depressing.

Antipyretic. - The salicylates lower febrile temperature.

Antirheumatic.-For this action the salicylates are mostly employed. They relieve the pain, lower the fever, and slow the pulse.

Therapeutic Indications.

As an anhidrotic the acid is used as a dusting-powder for sweating feet and perspiring axillæ.

As an antiseptic the salicylates and salol are used in gastric fermentation, flatulent dyspepsia, typhoid fever, and diarrhea.

As an antipyretic in rheumatism and influenza.

As an antirheumatic and antineuralgic they are most frequently employed, being very valuable in rheumatism, neuralgia, migraine, and tonsillitis.

Salicylic acid is much used for its power of removing exuberant tissue, as corns, callosities, warts, and in scaly eczema and psoriasis.

Administration.-The salicylates and salicylic acid, 
on account of their disagreeable taste, should be given in capsules, konseals, or tablet form.

In rheumatism any of the salicylic compounds are useful. Sodium bicarbonate, potassium citrate, or some alkaline salt should be combined with the salicylate to aid in its action and to prevent cardiac complications.

As an intestinal antiseptic, salol is preferrable. In neuralgic conditions, salicin, salophen, or salol are good.

Pretoxic Signs.-Ringing in the ears, fulness of the head, headache, and obtunded hearing.

Toxicology.-During the use of the salicylic compounds a set of symptoms may occur to which the term salicylism is applied. They are flushed face, nausea, dyspepsia, deafness, dimness of vision, weak pulse, and delirium. The writcr has had small doses of sodium salicylate produce great delirium in rheumatic patients.

SANTAL WOOD $(*)$

SANTONIN $(*)$

SAVIN (*)

SCAMMONY $(*)$

SCOPARIUS

Derivation.-The tops of Cytisus Scoparius.

Synonym.-Broom Top.

Constituent.-Spartein.

Dosage.-10-6o grains.

Preparations.

Fluidextract.-Dose, Io-60 minims.

Spartein Sulphate.-Dose, $\frac{1}{8} \frac{1}{2}$ grain. 


\section{Physiologic Action.}

Diuretic.-Scoparius taken internally acts upon the kidneys and increases the amount of urine.

Cardiac Stimulant.-Spartein represents the heartstimulating part of scoparius. It acts especially on the nervous mechanism of the heart, causing a slow, forcible heart action.

Therapeutic Indications.

As a diuretic in dropsy of cardiac origin.

As a stimulant in heart affections due to disturbance of the nervous mechanism, as palpitation, tobacco heart, coffee- and tea-drinker's heart.

SENEGA $(*)$

SENNA $(*)$

\section{SILVER NITRATE}

Synonyms.-Argenti Nitras, Lunar Caustic.

Dosage. $-\frac{1}{8}-\frac{1}{2}$ grain.

Preparations.

Dilute Silver Nitrate.

Fused Silver Nitrate.-Lunar caustic stick.

Action and Use.

Astringent and Caustic.-Applied externally silver nitrate causes an astringing effect, followed by destruction of tissue. It is used for these actions in stopping bleeding and for removing warts and vegetations.

Antiseptic.-Silver nitrate in dilute solution is a strong antiseptic, and is employed in gonorrheal urethritis and ophthalmia neonatorum.

Intcrnally silver nitrate is used in gastritis, gastric ulcer, chronic diarrhea, and epilepsy. 
Administration.-In gastric diseases this drug should be given when the stomach is empty, and followed by a large draught of water.

Toxicology.-Acute Poisoning.-The symptoms are burning pain in the abdomen, vomiting, catharsis, spasms, and collapse.

Management.-Give emetics, especially using salt water. Give white of egg, milk, and broths.

Chronic Poisoning.-This is termed Argyria, and is characterized by dyspepsia, gray discoloration of the skin and mucous membranes, anemia, and ulcerations.

\section{SODIUM COMPOUNDS}

\section{SODIUM ARSENATE}

Same action as Arsenic (which see). Dose, $\frac{1}{40}-\frac{1}{20}$ grain.

\section{SODIUM BENZOATE}

See Benzoin Compounds. Dose, 5-60 grains.

\section{SODIUM BICARBONATE}

Synonyms.-Saleratus, Baking Soda.

Dosage.-IO-I 20 grains.

Action and Use.

Given before meals it increases the flow of the gastric juice, given after meals it neutralizes the acid of the gastric secretion. It is used after meals for acid gastritis, pyrosis, and vomiting. This salt tends to render the urine alkaline, and is used in cystitis.

Externally it is a sedative, and is employed in burns and for itching. 
SODIUM BORATE

Also known as Borax. (See Boric Acid.)

\section{SODIUM BROMID}

Same action and uses as potassium bromid, except that the sodium salt is less irritating. Dose, Io-60 grains.

\section{SODIUM CACODYLATE}

This is an arsenical derivative, and is indicated in the same conditions as arsenous acic. Dose, $\frac{1}{2}-2$ grains.

\section{SODIUM HYDRATE}

Synonyms.-Caustic Soda, Sodium Hydroxid, Soda. Action and Use.

Applied externally this preparation is a strong irritant and caustic. It is but little used as a caustic.

\section{SODIUM IODID}

Same action and uses as Potassium Iodid (which see). Dose, 5-60 grains.

\section{SODIUM NITRITE}

Same action and uses as Nitroglycerin, except the action of the nitrite is slower and more prolonged. Dose, $\frac{1}{2}-2$ grains.

\section{SODIUM PHOSPHATE}

This salt is considered under Phosphorus Compounds.

SODIUM SALICYLATE

See Salicylic Acid Compounds. Dose, 5-30 grains. 
SODIUM SULPHATE

Synonym.-Glauber's Salt.

Dosage.-I -8 drams.

Sodium sulphate is a strong saline cathartic.

\section{SODIUM SULPHITE}

This drug is used as a gastro-intestinal antiseptic in gastric fermentation and also employed locally in parasitic skin diseases.

Dosage. $-5-30$ grains.

\section{SODIUM THIOSULPHATE}

Synonym.-Sodium Hyposulphite.

The action of this drug is the same as that of the sulphite, and is used in the treatment of parasitic skin diseases.

\section{SQUILL}

Derivation.-The bulb of Urginea Maritima.

Synonyms.-Scilla, Sea Onion.

Dosage.-I-5 grains.

Preparations.

Fluidextract.-Dose, I-5 minims.

Tincture.-Dose, 5-20 minims.

Vinegar.-Dose, 5-40 minims.

Syrup.-Dose, 15-60 minims.

Compound Syrup.-Dose, 5-30 minims. Also known as Hive Syrup. (See under Antimony and Potassium Tartrate.)

Physiologic Action.

Gastro-intestinal Irritant.-Squill in moderate doses produces nausea and vomiting. 
Expectorant.-This drug is a powerful expectorant, causing increase of the bronchial mucus.

Cardiac Stimulant.-Squill acts very much like digitalis on the heart, producing a slow, powerful heart action.

Diuretic.-The kidneys are irritated and their vascularity increased by this drug, thus the flow of urine is increased.

Therapeutic Indications.

As an expectorant in the latter stage of acute bronchitis, chronic bronchitis, and laryngitis.

As a diuretic in cardiac dropsy, chronic nephritis, and fluid accumulations of the pleural and pericardial cavities.

\section{STRAMONIUM $\left(^{*}\right)$}

\section{STRONTIUM SALTS}

The strontium salts used in medicine are the lactate, bromid, salicylate, and iodid. The last three have the same action and uses as the corresponding sodium salts.

Strontium lactate is said to decrease the amount of albumin in the urine of nephritis. Dose, 5-20 grains.

\section{STROPHANTHUS}

Derivation.-The seeds of Strophanthus Hispidus.

Constituent.-Strophanthin, a glucosid.

Preparations.

Tincture.-Dose, 2-10 minims.

Strophanthin.-Dose, $\frac{1}{200}-\frac{1}{100}$ grain. 
Action and Use.

This drug has the same action on the heart and kidneys as digitalis, except that it does not contract the vessels like digitalis. The uses are the same.

\section{SULPHURIC ACID}

Synonym.-Oil of Vitriol.

Properties.-A colorless, oily liquid, which is exceedingly corrosive. It contains 93 per cent. of absolute sulphuric acid.

Preparations.

Dilute Sulphuric Acid.-Dose, 5-20 minims, well diluted.

Aromatic Sulphuric Acid (Elixir of Vitriol) contains 20 per cent. of sulphuric acid and ginger and cinnamon. Dose, 5-20 minims.

Action and Use.

Externally the acid is an irritant and caustic.

Internally it checks the flow of gastric juice and acts in the intestines as an astringent.

It is used externally as a caustic to remove warts and small growths, and also as a dilute lotion for sweating. Internally it is employed in diarrhea.

Toxicology.-See Hydrochloric Acid.

\section{SUPRARENAL SUBSTANCE}

The active principle of the suprarenal, adrenal, or epirenal gland or capsule is a very useful medicinal agent.

Form.-This remedy is placed before the medical profession in numerous forms: As a dry or desiccated gland; as an extract of the gland; in the form of its 
principal constituent under one of many names, as adrenalin, suprarenalin, adnephrin, epinephrin, and hemostatin.

Dosage.-Dried gland, 3-8 grains; Extract, $\frac{1}{4}-1$ grain; Adrenalin (I : IO00 solution), 5-1 5 minims.

Physiologic Action.

Applied locally, solutions of the gland will produce great constriction of the vessels of the mucous membranes or the broken skin, thus causing paleness of the parts; later, a secondary relaxation of the vessel walls takes place.

Given internally the drug also causes a constriction of the vessels and a great rise of blood-pressure. The power and force of the heart is increased.

Therapeutic Indications.

Locally in capillary hemorrhage and to render operations on the eye, nose, throat, and other parts bloodless. To check secretions and lessen turgescence in hay-fever, rhinitis, etc.

Internally as a stimulant in shock, collapse, and cardiac failure.

\section{TERPIN HYDRATE $\left(^{*}\right)$}

THYMOL $\left({ }^{*}\right)$

Action.

\section{THYROID GLAND}

Given internally thyroid extract produces, in large doses, a rapid heart, dyspnea, frontal headache, and trembling. A continuous use of the drug will produce anemia, loss of weight, and weakness.

Uses.

This gland is employed extensively in the treatment of cretinism, myxedema, simple goiter, and obesity. 
It is also useful in skin diseases, fractures, and slow union of bone.

Dosage.-Dried gland, I-4 grains; Extract of the gland, $\frac{1-1}{4}$ grain.

\section{TRIONAL (*)}

\section{TURPENTINE OIL}

Derivation.-An oil distilled from the oleoresin of turpentine. It is a volatile, clear oil with a pine-like odor.

Dosage.-5-30 minims.

Preparations.

Rectified Oil.-Dose, 5-30 minims.

\section{Liniment.}

Physiologic Action.

Rubefacient.-Turpentine applied externally is irritating and produces redness of the skin.

Antiseptic.-Externally and internally oil of turpentine will retard bacterial growth.

Stimulant.-Reflexly from the stomach this drug stimulates the heart. It also stimulates intestinal peristalsis.

Anthelmintic.-The various intestinal worms are killed or their expulsion effected by turpentine in moderate doses.

Diuretic.-Turpentine irritates and causes congestion of the kidneys, thus increasing the output of urine. It may even produce bloody urine and partial suppression of this excretion. It imparts a violet-like odor to the urine.

Expectorant. - This drug acts as a powerful expectorant. 
Hemostatic.-By its action on the musculature of the blood-vessels it produces vascular constriction.

Therapeutic Indications.

As a rubefacient in rheumatism, pleurisy, bronchitis, and neuralgias.

As an antiseptic in cleansing ragged wounds and ulcers. Internally in typhoid fever.

As an intestinal stimulant in typhoid fever, pneumonia, and other conditions accompanied by tympanities.

As a hemostatic in gastric, intestinal, and uterine hemorrhage.

Administration.-As a relief for tympanities turpentine stupes are a most useful form of administration, also as enemata.

When giving turpentine either internally or externally a careful watch for toxic signs must be kept.

Turpentine is best given in capsules, as an emulsion, or dropped on sugar.

Toxicology.-Signs of poisoning are vomiting, purging, languor, unsteady gait, suppression of urine, hematuria, skin eruption, and collapse.

Management.-Empty the stomach and give demulcents, as gruels, flaxseed-tea, slippery elm solution. Stimulate if necessary.

\section{UVA URSI (*)}

\section{VALERIAN}

Derivation.-The root of Valeriana Officinalis.

Dosage.-IO-30 grains.

Preparations.

Fluidextract.-Dose, Io-30 minims.

Tincture.-Dose, $\frac{1}{4}-1$ dram. 
Ammoniated Tincture.-Dose, $\frac{1}{4}-1$ dram.

Ammonium Valerianate.-Dose, I-5 grains.

Iron Valerianate.-Dose, 1-3 grains.

Quinin Valerianate.-Dose, I-IO grains.

Sodium Valerianate.-Dose, I-5 grains.

Zinc Valcrianate.-Dose, $\frac{1}{2}-2$ grains.

Physiologic Action.

Gastric Stimulant.-All valerian preparations increase the motor and secretory actions of the stomach and promote the appetite and digestion.

Nerve Sedative.-Valerian tends to quiet nerve unrest and to relieve functional nervous excitability, for which purpose it is much used.

Therapeutic Indications.

The chief use of this drug is as a nerve sedative in such conditions as hysteria, nervousness, nervous insomnia, hypochondriasis, and the nervous disorders of pregnancy and the menopause.

\section{VERATRUM VIRIDE}

Derivation.-The rhizomes of Veratrum Viride.

Synonyms.-American or Green Hellebore.

Constituents.-Veratroidin and jervin.

Dosage.-I-3 grains.

Preparations.

Fluidextract.-Dose, I-3 minims.

Tincture.-Dose, 5-I 5 minims (U.S. P., I900).

Physiologic Action.

General Depressant.-This drug exerts a depressing action on the spinal cord; on the vasomotor system; on the heart, causing a weak and slow pulse. 
Therapeutic Indications.

Veratrum viride is the best heart sedative for use in the adult, being employed for the same purposes in the adult as aconite is for children. It is useful in the beginning of acute infectious fevers when the pulse is full and bounding, as in pneumonia, erysipelas, and pyemia. It is also invaluable in the treatment of puerperal eclampsia, being given until the pulse is reduced to about 80 beats per minute.

Pretoxic Signs.-Slow pulse, nausea, and vomiting.

Toxicology.-Although this is a most virulent drug, yet it is said that but few fatal cases of poisoning have resulted from its use. This is probably due to the emetic action of the drug, which eliminates it from the body when taken in toxic doses.

Signs of poisoning are nausea, vomiting, weak pulse, labored respirations, and cold, moist skin.

Management.-Empty the stomach and stimulate.

\section{VIBURNUM $(*)$}

\section{ZINC COMPOUNDS}

\section{ZINC CARBONATE}

In the impure state this preparation is known as Calamine, under which name it is much employed in irritating skin diseases.

\section{ZINC CHLORID}

A white, granular powder, which is very poisonous and caustic. It is also known as Zinc Butter. Used externally only. 


\section{ZINC OXID}

A white, amorphous, tasteless, and insoluble powder. It is employed mostly in the form of an ointment.

\section{ZINC SULPHATE}

Also known as White Vitriol. Dose, $\frac{1}{4}-\mathrm{I}$ grain.

Physiologic Action of the Zinc Salts.

Caustic.-Zinc chlorid destroys tissues to which it is applied.

Astringent.-The sulphate especially acts as an astringent, both externally and internally.

Emetic.-Zinc sulphate is a rapid and reliable emetic, causing vomiting by its irritant action on the stomach walls.

Therapeutic Indications.

As a caustic in the treatment of warts, nevi, lupus, condylomata, and epitheliomata.

As an astringent, externally for ulcers of the indolent type, gonorrhea, leukorrhea, and moist skin diseases. Internally in diarrhea and night-sweats.

As an emetic in drug poisoning.

Toxicology.-Nausea, vomiting, abdominal colic, purging, and collapse.

Management.-Wash out the stomach. Give tannic acid and demulcents, as white of egg, milk, lime-water. Stimulate and give opium for pain.

\section{OTHER ZINC PREPARATIONS}

Zinc Bromid.-Same action and uses as Potassium Bromid. Dose, $\mathrm{I}-3$ grains.

Zinc Iodid.-Same action and uses as Potassium Iodid. Dose, I-3 grains. 
Zinc Permanganate.-Same action and uses as Potassium Permanganate.

Zinc Phosphid:-See under Phosphorus. Dose, $\frac{1}{20}-\frac{1}{8}$ grain.

Zinc Sulphocarbolate (Zinc Phenolsulphonate).See Carbolic Acid. Dose, I-3 grains.

Zinc Valerianate.-See Valerian. Dose, $\frac{1}{2}-2$ grains. 


\section{PART III}

\section{Drugs of Minor Importance}

Absinth (Wormwood, Absinthium).-Used as a stomachic in gastric atony and dyspepsia. It is also an anthelmintic. Fluidextract, I5-45 minims.

Acacia (Gum Arabic).-Used as a demulcent in the form of a mucilage.

Adonis Vernalis.-The action of this drug is similar to digitalis, producing a slow and powerful heart action and diuresis. Fluidextract, I-2 minims; Adonidin, $\frac{1}{10}$ $\frac{1}{5}$ grain.

Agaricin.-A derivative of agaric. It decreases all secretions and is used with good results in night-sweats. Dose, $\frac{1}{4}$ I grain.

Allspice (Pimenta).-Acts as a stomachic and carminative in dyspepsia, colic, and anorexia. Fluidextract, 5-30 minims.

Ammoniac.-A gum resin having expectorant and antispasmodic properties. Used in bronchitis, asthma, and also as an emmenagogue in amenorrhea. Dose, 5-I 5 grains.

Anise.-An aromatic and carminative. Used much to relieve flatulence and colic in children. Powder, 520 grains ; Oil, I-IO minims. 
Apiol.-An oily liquid derived from parsley and used as a diuretic and emmenagogue. Dose, I-5 minims.

Apocynum (Canadian Hemp).-A useful diuretic much employed in dropsy. Extract, $\frac{1}{2}-2$ grains; Fluidextract, 2-10 minims.

Arnica.-Used externally in bruises and sprains, and internally as a diuretic and diaphoretic. Fluidextract, 5-15 minims; Extract, I-3 grains; Tincture, 15-60 minims.

Asafetida.-This drug is much used as a carminative, nerve sedative, and expectorant. It is excellent to relieve flatulence and colic of infants, being given as an enema. Also used in tympanities of typhoid fever, in nervous disorders of the functional type, and bronchitis. Tincture, $\frac{1}{4}-\mathrm{I}$ dram; Emulsion, I-4 drams.

Aspidium (Male fern, Filix mas).-Used extensively as an anthelmintic for tapeworms. Castor oil should not be administered when this drug has been given, as poisoning may result from absorption facilitated by the oil. Oleoresin or Extract, I-2 drams.

Aspidosperma.-Used in dyspnea, asthma, and bronchitis. Fluidextract, $\frac{1}{4}-\mathrm{I}$ dram.

Balsam of Peru.-Used externally as a stimulant to indolent granulations and ulcers. As an expectorant in asthma, chronic bronchitis, and pulmonary tuberculosis. Dose, 5-1 5 minims.

Barium Chlorid.-This salt has a similar action to dig. italis, producing a slow and forcible heart. It is used as an alterative in syphilis and scrofula. Dose, $\frac{1}{10}-\frac{1}{4}$ grain.

Blue Cohosh (Caulophyllum).-Used as an emmenagogue and as a relief for dysmenorrhea. Fluidextract, IO-30 minims; Caulophyllin, I-5 grains. 
Boneset.-This is more or less of a favorite diaphoretic with the laity, and is employed to abort "colds," bronchitis, and acute fevers. Fluidextract, I-2 drams.

Buchu.-The principal action of this drug is its sedative action on the urinary passages, for which purpose it is used in cystitis and urethritis. It is also employed as a diurctic in dropsy. Fluidextract, $\frac{1}{4}-\mathrm{I}$ dram.

Cactus (Night-blooming Cereus, Cereus Grandiflorus).-Cactus is a most valuable heart stimulant, increasing both the rate and force of the heart, and is used in palpitation and as a substitute for digitalis. The drug also possesses diuretic power. Fluidextract, 2-5 minims.

Calamus (Sweet Flag).-This drug is chiefly used as a carminative, and as such acts well in post-operative and fermentative flatulence. Fluidextract, 5-3? minims.

Calumba (Columbo). - This drug is one of the best bitters and stomachics and, as a rule, is acceptable to patients. Fluidextract, 5-20 minims; Tincture, I-2 drams.

Chamomile, German.-This drug is used as a carminative in flatulence and colic; as an antispasmodic in spasms of teething; as a diaphoretic in aborting "colds" and stimulating menstruation. Fluidextract, I 5-60 minims.

Cantharides (Spanish Fly).-Externally this drug acts as a mefacient and vesicant, and is used as such in neuralgias, meningitis, and pleurisy. Internally it is used as a diuretic and vesical stimulant. Tincture, I5 minims; Ccrate and Collodion, for external use.

Cardamom.-Used principally as a caminative and 
flavoring agent. Fluidextract, 5-15 minims; Compound Tincture, freely.

Cassia (Cinnamon).-This is used as a flavor. Carminative and astringent in dyspepsia, flatulence, and diarrhea. Fluidextract, IO-20 minims; Oil, I-3 minims.

Catechu (Gambir).-This drug is a powerful and valuable astringent, both externally and internally. It is useful locally in tonsillitis, pharyngitis, spongy gums, gonorrhea, and leukorrhea. Internally in diarrhea. Compound tincture, $\mathrm{I}-2$ drams.

Caulophyllum.-See Blue Cohosh.

Celery (Apium Graveolens).--The seeds are used as a carminative and nerve stimulant. Fluidextract, 5-15 minims.

Cerium Oxalate.-This metallic preparation is principally used as an anti-emetic in vomiting of pregnancy, seasickness, and dyspepsia. It is said to be a cough sedative of some value, but as such the writer has seen no beneficial results. Dose, I -5 grains.

Chloralamid.-This preparation is extensively employed as an hypnotic in insomnia not accompanied by pain. Dose, 15-40 grains.

Chloralose.-This is another hypnotic. Dose, 3-10 grains.

Chrysarobin (Goa Powder). - This is a most excellent application in the treatment of psoriasis and other skin diseases. Used as an ointment or with collodion. This drug should not be applied near the eyes.

Cimicifuga (Black Cohosh, Black Snakeroot).-Acts as an analgesic, antispasmodic, and heart depressant. It is useful in chorea, rheumatism, neuralgia, asthma, 
and dysmenorrhea. Fluidextract, $15-45$ minims; Extract, $\mathrm{I}-5$ grains.

Cinnamon.-See Cassia.

Citric Acid.-Used as a remedy for scurvy. Dose, 5-20 grains.

Cloves (Caryophyllus).-Used as a carminative and flavor. Fluidextract, 5-20 minims; Oil, 1-5 minims.

Cocculus.-Important because of its principal constituent, Picrotoxin, which is employed in night-sweats. Dose, $\frac{1}{60}-\frac{1}{15}$ grain.

Colocynth.-This drug is a powerful hydragogue cathartic. It is always used in combination with other drugs. Dose, I-8 grains. Extract, $\frac{1}{2}-2$ grains; Colocynthin, $\frac{1}{8}-\frac{1}{3}$ grain.

Conium (Hemlock). - This drug is a motor depressant and is used in chorea, neuralgias, convulsions, and asthma. Dose, I-3 grains ; Extract, $\frac{1}{2}-2$ grains ; Fluidextract, I-5 minims; Coniin, $\frac{1}{60}-\frac{1}{20}$ grain.

Copaiba.-Its chief uses are as an cxpctorant and urinary stimulant. Used in bronchitis, cystitis, gonorrhea, and leukorrhea. Oil, 5-10 minims; Mass, 5-45 grains ; Rcsin, 5-10 grains.

Copper Compounds.-The arscnite is used in anemia, diarrhea, cholera infantum, typhoid fever, and dysentery. The sulphate is employed locally as an astringent and caustic in the treatment of ulcers, gonorrhea, warts, trachoma, and oozing of blood. Internally as an cmetic and astringent. Arscnitc, $\frac{1}{200} \frac{1}{100}$ grain; Sulphate, $\frac{1}{6}-2$ grains.

Corn-silk (Zea Mays).-Used as a diuretic and sedative to the urinary tract. Employed in cystitis, lcukorrhea, and gonorrhea. Fluidextract, $\frac{1}{2}-2$ drams. 
Couch Grass (Triticum).-Uses and dosage the same as for Corn-silk.

Creolin is a deodorant and antiseptic. Used as an irrigant and douche in washing out cavities, and in cystitis, leukorrhea, and gonorrhea. With water it forms a milky and soapy solution. Employed in solution of $\frac{1}{2}-2$ per cent.

Croton Chloral (Butyl Chloral Hydrate).-Used as an analgesic, especially in neuralgia of the fifth cranial nerve or tic doloreaux. Dose, $2-5$ grains, given every half hour until relief is obtained; or 15 grains in one dose.

Cubebs acts as a diuretic, cxpectorant, and vesical stimulant. Used in chronic bronchitis, pharyngitis, cystitis, and urethritis. Fluidextract, Io-60 minims; Oil, 5-10 minims; Oleoresin, 5-20 minims; Tincture, I-2 drams.

Cusso (Kousso).-An excellent anthelmintic in the treatment of tapeworm. Dose, I-4 drams; Fluidextract, I-4 drams.

Duboisin.-Used exclusively as a mydriatic and nerve sedative. It is employed largely in place of atropin, as the former is more rapid in its action, less irritating, and more transient. As a nerve sedative it is used to quiet insane patients. Dose, $\frac{1}{10} \frac{-1}{40}$ grain.

Dulcamara (Bitter Sweet).-Said to be an alterative, diurctic, and diaphoretic, but is seldom used.

Echinacea.-Although an old drug, it has lately come to the front again, is much lauded as a powerful and efficient alterative in syphilis, and is very beneficial in the treatment of septic wounds, septicemia, and pyemia. Fluidextract, 10-30 minims. 
Elaterium.-A powerful hydragogue cathartic, very useful in rapidly producing free bowel evacuations and in reducing dropsical accumulations. It is best given as Elaterin, dose, $\frac{1}{30-\frac{1}{15}}$ grain. Dose of elaterium is $\frac{1}{10}-\frac{1}{2}$ grain.

Erigeron Oil (Oil of Fleabane).-This drug is useful in stopping oozing of blood in hematuria, menorrhagia, hematemesis, etc. Dose, 5-Io minims.

Eriodictyon (Yerba Santa).-A very agreeable expectorant in bronchitis and asthma. On account of its taste it is used to cover the bitterness of quinin. Dose, 15-60 grains. Fluidextract, 15-60 minims; Aromatic Syrup, I-3 drams.

Ethyl Bromid.-A clear, volatile, and inflammable liquid, used locally as an anesthetic. It is also employed as a general anesthetic.

Ethyl Chlorid.-A local and general anesthetic.

Eucain.-A substitute for cocain as a local anesthetic. Used as a 2-Io per cent. solution.

Eucalyptus is an antiperiodic, antiseptic, and expectorant. Employed in malaria, asthma, and bronchitis. Oil, 5-IO minims; Fluidextract, $\frac{1}{4}-\mathrm{I}$ dram; Eucalyptol, 5-10 minims.

Euonymus (Wahoo).-Used principally as a cholagogue cathartic in jaundice, hepatitis, biliousness, and malaria. Extract, I-5 grains; Fluidextract, I-2 drams.

Eupatorium.-See Boneset.

Fennel.-Used as a carminative in flatulence and infantile colic. Fluidextract, 5-20 minims.

Frangula (Buckthorn).-A mild and pleasant laxative, used much in chronic constipation. Fluidextract, 15-30 minims; Extract, 5-I 5 grains. 
Gamboge.-An uncertain hydragogue cathartic, attended with griping. Dose, I-4 grains.

Gossypii Radicis Cortex (Cotton-root Bark) is an emmenagogue and oxytocic. Used in amenorrhea and dysmenorrhea. Fluidextract, 15-60 minims ; Extract, I-IO grains.

Grindelia is an expectorant and antispasmodic, used in asthma, whooping-cough, and hay-fever. The fluidextract is an excellent application for ivy-poisoning. Fluidextract, $\frac{1}{4}-\mathrm{I}$ dram.

Guaiac.-A diaphoretic and antirheumatic. Used in subacute and chronic rheumatism, tonsillitis, pharyngitis, amenorrhea, and dysmenorrhea. Dose, 5-I 5 grains. Tincture, $\frac{1}{4}-\mathrm{I}$ dram; Ammoniated Tincture, I2 drams.

Hamamelis (Witch Hazel).-Used mostly as an astringent and hemostatic in piles, diarrhea, leukorrhea, and gonorrhea. Fluidextract, $\frac{1}{4}-\mathrm{I}$ dram.

Helonias (False Unicorn Root).--Used as a uterine sedative and tonic. Fluidextract, $\frac{1}{2}-2$ drams.

Hematoxylon (Log Wood).-Used as an astringent in diarrhea, dysentery, and cholera infantum. Extract, 5-I 5 grains.

Humulus (Hops).-This drug acts as a nerve sedative and hypnotic. Fluidextract, $\frac{1}{4}-\mathrm{I}$ dram; Tincture, I-3 drams; Lupulin, 2-IO grains; Fluidcxtract of Lupulin, 2-1 5 minims.

Ingluvin (Extract of Chicken Gizzard).-Used as a digestant and anti-emetic. Dose, 5-20 grains.

Iris (Blue Flag).-A cholagogue cathartic, used in hepatic torpor and biliousness. Extract, I-4 grains; Fluidextract, 5-20 minims ; Irisin, $\frac{1}{2}-3$ grains. 
Jamaica Dogwood (Piscidia).-Nerve sedative and hypnotic. Fluidextract, $\frac{1}{4}-\mathrm{I}$ dram.

Juniper.-A stimulating dineretic, not to be used in acutely congested nephritis. Fluidextract, I-2 drams; Spirit, $\frac{1}{2}-1$ dram; Oil, 2-10 minims.

Kamala.-Used as an anthelmintic for tapeworm. Fluidextract, I-20 drams.

Kava Kava.-Useful in mucous-membrane inflammations, as bronchitis, cystitis, and gonorrhea. Fluidextract, $\frac{1}{4}$ - I dram.

Kino.-Very useful astringent in diarrhea. Tincture, $\mathrm{I}-3$ drams.

Kola (Cola).-Nerve sedative and astringent. Fluidextract, $\frac{1}{4}-\frac{1}{2}$ dram.

Krameria (Rhatany).-Strong astringent and hemostatic. Used in diarrhea, sweating, leukorrhea, and bleeding from the mucous membranes. Fluidextract, Io-30 minims; Tincture, I-3 drams ; Syrup, I-3 drams.

Lactucarium (Lettuce).-Hypnotic and nerve sedative. Fluidextract, 5-I5 minims.

Lanum (Lanolin, Hydrous Wool-fat).-Used as a base for ointments.

Leptandra (Culver's Root).-Cholagogue cathartic, used in constipation, jaundice, and hepatic torpor. Extract, 3-10 grains; Fluidextract, I 5-45 minims; Leptandrin, $\mathbf{I}-5$ grains.

Lobelia.-Used as an cxpectorant and antispasmodic in asthma, chronic bronchitis, whooping-cough, and spasmodic conditions. Fluidextract, 2-10 minims; Tincture, 10-30 minims; Lobelin Sulphate, $\frac{1}{2}-2$ grains.

Methylene-blue.-Used as an antiperiodic in malaria and as an antiseptic in the treatment of gonorrhea and 
cystitis. This drug causes the urine to become of a blue-green color. Dose, I-4 grains, in capsules.

Musk.-This drug is of animal origin. It acts as an antispasmodic and stimulant in low nervous conditions of the acute fevers. The drug is extremely expensive. Dose, $3-10$ grains ; Tincture, $\frac{1}{2}-1$ dram.

Musk Root (Sumbul).-Used exclusively as a nerve sedative in hysteria, nervousness, and insomnia. Fluidextract, 10-20 minims.

Mustard (Sinapis).-Acts as a rubefacient, counterirritant, and emetic.

Myrrh.-Astringent, carminative, and cmmenagogue. Useful for spongy gums, stomatitis, amenorrhea, and chlorosis. Tincture, I 5-60 minims.

Naphtol.-Used as an intestinal antiseptic in diarrhea, typhoid fever, cholera infantum, and enteritis. Dose: Alpha, I-8 grains; Beta, 5-I 5 grains.

Paraldehyd.-Used as an hypnotic. The taste is so disagreeable that it should be given well diluted in sweetened water. Dose, $\frac{1}{2}-\mathrm{I}$ dram.

Passion Flower (Passiflora).-Used as a nerve sedative in hysteria, nervousness, and insomnia. Fluidextract, $\frac{1}{4}-\mathrm{I}$ dram.

Phytolacca (Poke Root or Berry).-Used as an alterative in syphilis, rheumatism, and scrofula. Recently has been employed in obesity. Dose, I-5 grains. Fluidextract, I-5 minims.

Picrotoxin.-See Cocculus.

Prunus Virginiana (Wild Cherry).-Used as a cough sedative and expectorant. Fluidextract, $\frac{1}{4}-\mathrm{I}$ dram; Syrup, I-4 drams; Infusion, $\frac{1}{2}-2$ ounces. 
Pumpkin Seeds (Pepo).-Used as an anthelmintic for tapeworm. Dose, I ounce.

Quassia.-A bitter and anthelmintic. Used in anorexia, dyspepsia, and gastric atony. As an enema for seat- of pin-worms. Fluidextract, IO-30 minims; Tincture, I-3 drams; Infusion (as enema), I-2 ounces.

Saccharin (Glusid).-This is a coal-tar product with an intensely sweet taste. It is employed as a substitute for sugar in diabetic patients. One grain is equivalent in sweeting power to I ounce of sugar.

Saffron.-Used formerly as an antispasmodic and emmenagogue.

Sanguinaria (Blood Root).-Used as an expectorant in bronchitis, croup, and asthma. Fluidextract, 3I 5 minims; Tincture, $\frac{1}{4}-1$ dram; Sanguinarin, $\frac{1}{12}-\frac{1}{4}$ grain.

Santal Wood (Sandal Wood).-Used as an antiseptic and stimulant to the urinary tract in cystitis and gonorrhea. Fluidextract, $\frac{1}{4}-1$ dram; Oil, 5-20 minims.

Santonica (Levant Worm-seed).--Used as an anthelmintic for the round-worm. Santonin, $\frac{1}{4}-2$ grains.

Savin.-Emmenagogue in amenorrhea. Oil, I-5 minims.

Saw Palmetto.-Bronchial and urinary stimulant. Used in bronchitis, asthma, laryngitis, cystitis, and gonorrhea. This drug is said to produce an increase in weight and nutrition. Dose, $\frac{1}{2}-\mathrm{I}$ dram; Fluidextract, $\frac{1}{2}-\mathrm{I}$ dram.

Scammony.-Hydragogue cathartic, similar to jalap. Resin, I-5 grains. 
Senega acts as an expectorant and diuretic. Used in chronic bronchitis, asthma, croup, and dropsy. Fluidextract, 10-20 minims; Syrup, $\frac{1}{2}-2$ drams.

Senna.-Chief action is that of a laxative. Fluidextract, I-2 drams; Compound Infusion (Black Draught), I-2 ounces.

Spigelia (Pink Root).-Used as an anthelmintic for the round-worm. Fluidextract, I-2 drams.

Stavesacre (Staphisagria, Delphinum).-Used principally as an antiparasiticide. It is very poisonous.

Stramonium.-This drug is a powerful antispasmodic, belonging to the same class as belladonna and hyoscyamus. Used particularly in asthma by inhaling the vapors from the burning leaves. Fluidextract, I-5 minims ; Tincture, 5-1 5 minims.

Sulfonal.-Used as an hypnotic. Dose, Io-30 grains.

Sulfur.-Used as a disinfectant by burning. Internally it is a laxative. Precipitated Sulfur, $\frac{1}{2}-\mathrm{I}$ dram.

Terpin Hydrate.-Useful in chronic bronchitis, hayfever, and cystitis. Dose, 2-5 grains.

Thymol.-Antiseptic and anthelmintic. Used in typhoid fever, gastric fermentation, bronchitis, and coryza. It is also employed in the treatment of intestinal worms. Dose, I-IO grains.

Tolu.-Used as an expectorant. Syrup, I-4 drams. Trional.-Used as an hypnotic in insomnia not accompanied by pain. Dose, IO-30 grains.

Uva Ursi (Bear-berry).-Acts as a diuretic and stimulant to the urinary tract. Used in chronic nephritis, cystitis, and gonorrhea. Fluidextract, 1-I dram; Arbutin, I-3 grains. 
Viburnum Prunifolium (Black Haw).-Used principally as a uterine antispasmodic in dysmenorrhea, threatened abortion, and habitual miscarriages. Fluidextract, $\frac{1}{4}-\mathrm{I}$ dram.

Viburnum Opulus (Cramp Bark).-Used as a nerve sedative and antispasmodic in asthma, hysteria, and nervousness of women.

12 


\section{PART IV}

\section{Newer Medicinal Agents}

THis section refers to the newer definite chemic products and not to the multitude of medicinal mixtures, the ingredients of which are more or less undivulged.

Acetozone.-One of the organic peroxids, claimed to be a powerful germicide. It is soluble in water, is non-explosive, and does not corrode tissues or act toxic internally. It is used locally for ulcers, rhinitis, leukorrhea, and internally in typhoid fever, diarrhea, and fermentative dyspepsia.

Dosage.-Internally it is administered greatly diluted. Two grains or more of the drug are dissolved in a glass of water and this amount taken every two hours. Externally it is used as a dusting-powder, mixed with boric acid, talcum, or other inert powder, or a solution of $I: 2000$ is used as a lotion or douche.

Actol (Silver Lactate).-This is an organic silver salt. It is a white powder, soluble in 15 parts of water. It acts as an astringent and antiseptic. Like other silver salts, it is especially valuable in those conditions due to the gonococcus. It may be employed sub178 
cutaneously in erysipelas. Dose, $\frac{1}{4}-\frac{3}{4}$ grain. As an irrigant in a $\mathrm{I}$ : 2000 solution.

Agurin (Acetotheobromin Sodium). - A white powder, soluble in water. Used as a diuretic for reducing dropsical accumulations due to cardiac or renal disease. Dose, 7-1 4 grains.

Airol.-An iodin preparation of bismuth and gallic acid. Used as a dressing powder for ulcers, wounds, skin diseases, and affections of the nose, ear, and throat. It has been used internally for diarrhea in doses of from I-3 grains.

Albargin (Gelatose Silver).-An organic silver preparation containing 15 per cent. of silver and soluble in water. Used in gonorrheal affections.

Alphozone (Succinic Peroxid).-An organic peroxid somewhat similar to acetozone in action and uses.

Amyloform.-A combination of formaldehyd and starch. Used as a substitute for iodoform. It does not irritate or cause toxic symptoms.

Anesthol.-A clear and transparent liquid with an agreeable odor. It is used as an anesthetic, being a combination of ethyl chlorid, ether, and chloroform.

Argenol.-A combination of silver and an albuminous substance. Soluble in water and used as other organic silver combinations.

Argentamin.-A solution of Io per cent. of silver in organic combination. It is non-irritating, very penetrating, and stronger than silver nitrate. Used in gonorrheal diseases in solutions of I : 100 to I : 400 .

Argyrol.-A combination of silver and yelk of egg. Used in solution of $\mathrm{O} . \mathrm{I}-4$ per cent. strength. 
Aristochin.-A tasteless quinin preparation. Dose same as Quinin.

Aristol.-A derivative of thymol and iodin. Used as a dressing for wounds, ulcers, burns, etc. It should be protected from the light.

Benzosol.-A guaiacol-benzoin combination, containing 54 per cent. of guaiacol. Used as an intestinal antiseptic. Dose, I-Io grains.

Bromoform.-A bromin derivative, used especially in the treatment of whooping-cough. Dose, I-IO drops. Its use is not free from danger.

Chloretone.-A derivative of chloroform and acetone. Used as an hypnotic and anesthetic. Dose, 5-15 grains.

Collargol is a soluble metallic silver used in septic conditions, as puerperal fever, pyemia, erysipelas, and all infectious conditions. Administered intravenously, 30 minims of a 5 per cent. solution, well diluted; by rectum, 5 grains in I ounce of water; by mouth, tablets of $\mathrm{I}$ or 2 grains; local application, varying from $\frac{1}{2}-5$ per cent. solution. Unguentum Credé is a 15 per cent. ointment of collargol.

Diuretin.-A combination of sodium salicylate and theobromin. Used as a diuretic in nephritis and dropsy. It should be given as a fresh solution in water. Not more than six doses of 15 grains each should be given in twenty-four hours.

Dolomol preparations are combinations of various medicaments with the stearate of calcium and magnesium.

Dormiol is a combination of chloral and amylene hydrates. Used as an hypnotic. Dose, 5-20 grains. 
Eosote (Creosote Valerianate). - Used in tuberculosis and diarrheas. Dose, I-IO minims.

Euquinin.-A tasteless quinin. Use and dosage the same as Quinin.

Exalgin.-An acetanilid derivative, used the same as Acetanilid. Dose, I-4 grains.

Formin.-See Urotropin.

Geosote (Guaiacol Valerianate).-Used as an intestinal antiseptic and in tuberculosis. Dose, 3-ro minims.

Hedonal.- Used as an hypnotic in nervous insomnia. Best given in dry form or in capsules. Dose, 15-30 grains.

Helmitol.-Same action and use as Urotropin.

Holocain.-A substitute for cocain. Used in a I per cent. solution.

Hyrgol.-A colloidal preparation of mercury. Used in syphilis and said not to interfere with digestion. Used as an inunction.

Ichthalbin.-A combination of ichthyol and albumin. Used same as Ichthyol. Dose, 3-10 grains, internally.

Ichthargan.-A combination of ichthyol and silver, and contains 30 per cent. of silver. Used in gonorrheal disease in a $0.2-3$ per cent. solution.

Ichthoform.-A combination of ichthyol and formaldehyd. Used as an antiseptic both externally and internally. Dose, Io-30 grains.

Iodol.-An iodin derivative of 89 per cent. strength. Used as a substitute for iodoform.

Itrol (Silver Citrate).-Used the same as Actol.

Lycetol.-A compound of piperazin. Used in gout, lithemia, calculi, and chronic rheumatism. Dose, 4-10 grains. 
Lysol.-A brown oily liquid, very similar in action and uses to creolin. Used in $\frac{1}{2}-2$ per cent. solution.

Mesotan.-A salicylic acid derivative, used locally as an analgesic in rheumatism and neuralgias. Applied with an equal part of sweet oil.

Nosophen.-An iodin derivative of 60 per cent. strength. Used as a substitute for iodoform.

Orthoform. - A benzoic derivative with a local anesthetic action similar to cocain. Used for burns, fissures, and painful wounds.

Perhydrol.-A 30 per cent. solution of hydrogen peroxid.

Picratol.-A carbolic acid derivative of silver, containing 30 per cent. of silver. Used as other silver preparations.

Piperazin.-Used as a uric-acid solvent in gout, lithemia, and rheumatism. Dose, 5 grains three times a day, dissolved in plenty of water.

Proferrin.-A nucleoproteid combination of iron. Used in anemia. Dose, 5-10 grains.

Protan.-A nucleoproteid combination of tannic acid. Used in diarrhea. Dose, 5-30 grains.

Protargol.-A combination of proteid with 8 per cent. of silver. Used as other organic silver preparations in a $0.2-10$ per cent. solution.

Salipyrin (Antipyrin Salicylate).-Analgesic, antipyretic, and antirheumatic. Dose, 5-30 grains.

Stypticin (Cotarnin Hydrochlorate).-A derivative of an alkaloid of opium(Narcotin). Locally or internally it causes vasoconstriction. Dose, I-4 grains. Used in uterine hemorrhage, nosebleed, and hemoptysis. 
Sublamin.-A mercurial derivative used as an antiseptic in place of mercury bichlorid. It is non-irritating. Used in $\mathrm{I}: \mathrm{I} 1000$ to $\mathrm{I}: 500$ solutions.

Tannalbin.-A combination of albumin and tannic acid. Used as an astringent in diarrheas. Dose, IO60 grains.

Tannigen.-A tannic acid combination. Used as an intestinal astringent. Dose, 3-I 5 grains.

Tannocol.-A combination of tannic acid and gelatin. Dose, 7-I 5 grains.

Tannoform.-A combination of tannic acid and formaldehyd. Used. in skin diseases and for local sweating.

Theocin.-A powerful diuretic, used in doses of 3-5 grains.

Thiocol.-A guaiacol derivative, used in tuberculosis, coughs, and as an intestinal antiseptic. Dose, 5-20 grains.

Thiosinamin.-A tissue solvent. Used in lupus, tumors, old scars, and strictures. Hypodermically, I-3 grains, in a I 5 per cent. alcoholic solution.

Tricresol.-A mixture of three cresols. Used as a disinfectant in $\frac{1}{2}-\mathrm{I}$ per cent. solution.

Urotropin (Hexamethyleneamin).-A powerful urinary antiseptic. In the kidneys it sets formalin free, thus converting the urine into an antiseptic solution. Used in cystitis, pyelitis, nephritis, urethritis, and typhoid fever. Dose, 5-7 grains, dissolved in water and given three times a day, after meals. Hexamethyleneamin is now an official preparation.

Veronal.-A recent hypnotic, used in nervous insomnia. Dose, 5-1 2 grains, given in a hot drink. 
Xeroform.-A substitute for iodoform. It is free from odor, taste, and irritation. Used internally as an intestinal antiseptic. Dose, 5-15 grains.

Yohimbin.-An alkaloid from the bark of Yohimbehe, grown in West Africa. Used in impotence. Dose, $\frac{1}{15}$ grain. 


\section{PART V}

\section{Practical Therapeutics}

\section{HYDROTHERAPY}

\section{BATHS}

IN the treatment of acute febrile diseases baths are used for two chief purposes: To reduce the fever; to impress the nervous system. This latter purpose is fully as important as the reduction of the temperature. Baths are instituted in the treatment of fever when the temperature of the patient reaches a certain height, generally $103^{\circ} \mathrm{F}$, or when the nervous symptoms become marked. There are several varieties of baths, the best and most frequently employed methods are: Sponge-bath, tub-bath, bed-bath, and packs.

Sponge-bath.-This form of bathing is used when the fever is not excessive and is easily influenced, especially in the case of children and weak and nervous adults. The patient is placed on several thicknesses of blankets or on a rubber sheet. A sponge is moistened with water of the required temperature, which should be higher for the initial baths than for the subsequent ones; the moist sponge is now lightly applied 
to the patient's body so as to leave a thin film of water on the skin, and not poured on from a dripping sponge, as is often the case. By applying only a thin film of water evaporation takes place rapidly and the temperature of the body falls correspondingly. It is very important that the surface of the body be kept glowing by constant rubbing with the hands to facilitate evaporation and to stimulate the surface circulation. In robust adults a very large part of the surface may be manipulated, but in weak adults and children it is preferable to bathe only a small part of the body at a time. If, after the sponge-bath is completed, alcohol be poured into the palms of the hands and then applied to the patient's body, a very agreeable and beneficial result is obtained. When the sponge-bath is finished the patient is covered with a light sheet.

Tub-bath.-This form of bath is used when the temperature is excessive and the patient is robust. The question as to the temperature of the water at the beginning of the bath is to be solved by the physician in attendance. The water may be warm at first, to prevent the initial shock, and then gradually cooled by cold water or ice to about $80^{\circ} \mathrm{F}$, or the water may be employed at $80^{\circ} \mathrm{F}$. at the beginning. Many physicians insist that this initial shock is beneficial.

The patient is placed on a stretcher made for the purpose, and is lowerd into the tub, or is rolled on to a blanket and lowered into the tub. When in the water, friction should be constantly applied to the patient's body to maintain the surface circulation. As the bathing progresses the water, which is becoming 
warmer, should be cooled by adding cold water or ice. An ice-cap placed on the patient's head will prevent the sometimes troublesome cerebral hyperemia. Before and, if necessary, after the tub-bath a diffusible stimulant, as aromatic spirit of ammonia, spirit of ether, or spirit of vini gallici, may be given.

The temperature of the patient should not be reduced below $100^{\circ} \mathrm{F}$, as collapse may follow, because the temperature continues to fall several degrees after the bath is discontinued.

Bed-bath.-This form of bathing has the advantages of a tub-bath, and is devoid of the danger involved in handling the patient necessary in tub-bathing.

A large rubber sheet is placed under the patient, then two blankets are rolled up lengthwise into two bolsters; one of these rolls is placed under the rubber sheet on each side of and running parallel with the patient's body, in such a manner that he lies in the trough. A pillow is placed under the upper end of the rubber sheet and another pillow at the foot under the rubber sheet, thus a rubber bath-tub is improvised, with the patient in it. Water of the required temperature is now poured into the tub and the bath is continued as in a tub-bath. When the bath is completed, the water is allowed to flow into a pail which is held under the edge of the rubber cloth at the foot of the bed, where the pillow has been partially removed.

Packs.-These the writer considers one of the best methods of applying cold for the purpose of reducing the temperature of a fever patient. They are partial and complete. 
First, place a rubber sheet under the patient to prevent the bedclothing from becoming wet. Next, wring out a sheet in cold water and wrap it about the patient's body from the chin to the toes. As the sheet becomes warm, pour cold water on from a pitcher or, better, from a sprinkling can. In the partial form, only the chest or the chest and abdomen are wrapped in the wet sheet.

A word here about the ice-bag will not be out of place.

Ice-bag.-This small, inexpensive, easily obtained, and, as some say (they do not know its value), insignificant piece of apparatus the author considers as the most valuable means of applying cold to a limited area. Not only is it useful in reducing the temperature, but in combating inflammation, preventing exudations, and in overcoming pain, especially in pleurisy, pericarditis, peritonitis, appendicitis, articular rheumatism, and, in fact, any inflammatory pain. This method is not employed as much as it should be.

\section{HYPODERMOCLYSIS}

This is another and equally important division of hydrotherapy.

Indications. - The subcutaneous injection of normal saline solution is a most valuable therapeutic agent. In the writer's hands the most gratifying results have been obtained by the use of hypodermoclysis. It will be found useful in those conditions characterized by a loss of body fluids, as hemorrhage and cholera. In circulatory depression of shock, surgical collapse, overanesthetization, and acute fevers, as pneumonia and 
typhoid fever. As an aid in the elimination of toxins and poisons in sepsis, septicemia, uremia, and diabetic coma.

Methods.-First, let us consider the solution for injection. By a normal saline or an isotonic solution we mean one which holds about as much saline material as the plasma of the blood, which is about 0.6 per cent. If to I quart of sterile water $\mathrm{I} \frac{1}{2}$ drams of salt (sodium chlorid) be added we have approximately a 0.6 per cent. solution.

Apparatus.-The apparatus necessary is a glass funnel, sufficiently large to hold I quart, a rubber tube three feet long, and an aspirating needle. All parts must be absolutely sterile.

Site of Injection.-This may be any place where the subcutaneous cellular tissue is abundant, as below the breasts, the anterior abdominal wall, or the inner portion of the thigh.

Place the solution in the funnel and allow the water to escape until the air is forced from the tube and the needle and the apparatus is warm. Roll up the skin by pinching it between the thumb and index-finger of the left hand, then insert the needle as you would in giving a hypodermic injection, making sure that the end of the needle is under the skin and in the loose cellular tissue. The needle being in place, the solution is allowed to slowly enter the tissues by elevating the funnel. The absorption of the fluid may be facilitated by kneading the tumor formed in the skin. About 1 or 2 pints should be injected at one time. The temperature of the solution should be about $105^{\circ} \mathrm{F}$. 


\section{ENTEROCLYSIS}

This is literally a drenching of the bowels.

Indications.-In the treatment of diseases of the intestines; to supply fluid to the system ; to add heat to the body; to abstract heat from the body; to wash out poisons eliminated through the intestinal mucous membrane; to reduce intestinal obstruction.

Treatment of Diseases of the Bowels.-Much success has followed the use of enteroclysis in the treatment of cholera. The bowels are washed out with I to 3 liters of normal saline solution or, better, with a I per cent. solution of tannic acid. The solution should be allowed to flow in slowly from a fountain-syringe held one or two feet above the patient. Dysentery is also efficiently treated by this means, using a $I: 2500$ solution of quinin, which rapidly destroys the ameba. Silver nitrate solutions (I 5 grains to the pint) are useful in subacute cases. In infantile bowel trouble, washing of the bowels with normal saline solution is most beneficial. Enteroclysis is also valuable in chronic diarrhea.

To Supply Fluid.-Enteroclysis, or rectal infusion of saline solution, is of great value in the same conditions as hypodermoclysis: In hemorrhage, shock, uremia, nephritis, diabetic coma, and diarrheas. After surgical procedures, when thirst is great but water is not allowed by mouth, a rectal infusion of I pint of saline solution is invaluable.

To Add Heat.-In shock, collapse, algid malaria, and conditions accompanied with a subnormal temperature a saline rectal infusion with a temperature of $100^{\circ} \mathrm{F}$. will aid in the restoration of the normal body heat. 
To Abstract Heat.-In high fever a rectal irrigation with cold water is advised. It will abstract heat rapidly and from parts where most of the heat is stored, but the method is not without danger.

To Eliminate Poisons.-In toxic conditions, when the poisons are eliminated through the mucous membrane of the intestines, the toxic products may be rapidly removed from the body with a normal saline irrigation of the colon.

In Intestinal Obstruction.-The obstacle may in some cases be removed by filling the bowel with saline solution under slight pressure, but with extreme care.

In conclusion, the writer wishes to state that in using large quantities of hot saline solution in the bowel you may raise the temperature of the body sufficiently high to produce heat-stroke.

\section{GASTRIC LAVAGE}

It is frequently necessary to wash out the stomach, especially in drug poisoning, diseases of the stomach, and in persistent vomiting. For this last purpose the writer has seen some excellent results in emesis after operations.

An ordinary stomach-tube with the funnel end is used. The tube is lubricated with warm water, not oil, glycerin, or vaselin, and then passed by directing the extremity well back toward the posterior pharyngeal wall and into the esophagus, when the patient is told to swallow, and the tube is passed into the stomach. If the stomach is empty, warm water is poured into the funnel and allowed to flow into the stomach. When 4 or 6 ounces of water have entered the organ 
and before the funnel is empty the outer end is lowered into a basin placed on the floor, when the stomach is emptied by siphonage. This is repeated several times. A small amount of water may be allowed to remain in the stomach. In removing the tube have the lumen closed by pinching the tube between the fingers, thus preventing water escaping from the tube and entering the larynx, which would cause coughing and disagreeable symptoms.

\section{HYPODERMIC MEDICATION}

This is the most accurate method of administering drugs. If a drug be given by the stomach, the amount which is absorbed varies between wide margins depending on the condition of the stomach and its contents. Some drugs are altered while in the stomach. If a drug be given to a person hypodermically you can depend on a rapid and accurate action of the same. The dose of drugs when given subcutaneously is less than the dose by mouth.

A description of the hypodermic syringe is unnecessary. The number of different syringes on the market is legion. Many of them are good.

Site of Injection.-Any part of the cutaneous surface where the skin is thin and soft may be selected. If the skin be thick the procedure is painful to the patient, and during the manipulations the needle may be broken or bent. The site should be cleansed with alcohol on a soft and clean cloth.

Method.-The needle is placed in a large iron or agateware spoon containing water and vigorously boiled for a minute or two over a lamp or gas flame, then 
draw the barrel of the syringe full of the hot water and throw away what water remains in the spoon, after having carefully removed the needle. Place the hypodermic tablet in the same spoon and eject upon it the water from the syringe, when the tablet will soon dissolve; then fill the syringe with this medicated solution and screw on the needle. Before giving the injection point the needle upward, and slowly press on the piston until a drop of the solution appears at the point of the needle and the air is expelled.

As said before, the injection may be given where the skin is thin and soft, as the inner surface of the arm toward the anterior or posterior aspect, the inguinal region, or the upper and inner part of the thigh. The skin is folded between the thumb and index-finger of the left hand, and with the right hand the needle is thrust through the skin near the base of the fold into the subcutaneous tissues, and the solution slowly injected.

One of the dangers of hypodermic injections is that a vein may be entered and the drug would be carried to the vital centers in a concentrated form. Abscesses occasionally follow an injection, but in a majority of instances are due to carelessness.

The more common drugs given hypodermically are: Aconitin, $\frac{1}{200}-\frac{1}{100}$ grain; apomorphin hydrochlorate, $\frac{1}{20}-\frac{1}{10}$ grain; atropin sulphate, $\frac{1}{200}-\frac{1}{50}$ grain; cocain hydrochlorate, $\frac{1}{10}-\frac{1}{6}$ grain; codein phosphate, $\frac{1}{8}-\frac{1}{2}$ grain; corrosive sublimate, $\frac{1}{100}-\frac{1}{30}$ grain; digitalin, $\frac{1}{100-\frac{1}{50}}$ grain; hyoscyamin (crystalline), $\frac{1}{12} \frac{1}{50}-\frac{1}{50}$ grain ; hyoscin hydrobromate, $\frac{1}{200}-\frac{1}{100}$ grain; morphin, $\frac{1}{8}-\frac{1}{4}$ grain; nitroglycerin, $\frac{1}{20}-\frac{1}{50}$ grain; pilocarpin hydrochlorate, $\frac{1}{20-\frac{1}{8}}$ grain; strychnin, $\frac{1}{100}-\frac{1}{20}$ grain. 


\section{ANTISEPTICS AND DISINFECTANTS}

Bichlorid of Mercury.-This is the most used of the stronger antiseptics. It is employed in surgery to disinfect the surgeon's hands and the site of operation. It is used to render sterile rubber and glass tubing, towels, and dressings. It is employed to irrigate wounds, purulent cavities, the vagina, etc. In dressing infected wounds it is very useful as a wet application.

It is employed in solutions of varying strengths, from I : I 5,000 to I : 500 . For use on the surgeon's hands and the site of operation in a I : 1000 solution; for irrigating cavities in a $1: 10,000$ solution; for disinfecting bedclothing from the beds of patients suffering from the acute contagious fevers a I : 500 solution is proper.

Decomposition of the bichlorid solution which often occurs may be prevented by adding to each pint of solution 4 grains of citric acid, or 8 grains of ammonium chlorid, or 8 grains of common salt, or 8 grains of tartaric acid.

Solutions of bichlorid of mercury cause corrosion of metallic substances, and should not be used to disinfect metal instruments or kept in metallic receptacles.

When this antiseptic is used as a moist dressing on wounds or as an irrigant for cavities, vigilance must be kept for the appearance of toxic signs, which may result from absorption of the drug. The first symptoms are salivation, abdominal colic, diarrhea, vomiting, sore gums, and ill-smelling breath.

Mercurial eczema occurs in nurses who are especially susceptible and have their hands immersed much in bichlorid solutions. 
Carbolic Acid.-Excepting the bichlorid of mercury this is the most employed of the strong antiseptics. It is used in surgery for sterilizing instruments and for cleansing surfaces.

It is employed in solutions varying from $I$ : Ioo to I : IO. A I : 20 solution is sufficiently strong for disinfecting instruments; for irrigating purposes, a I : IOO solution; for disinfecting bedclothing, a I : Io solution.

Like bichlorid of mercury, carbolic acid when applied externally may be absorbed and produce such toxic signs as headache, vertigo, nausea, dark-colored urine, and purging. Gangrene has followed the local use of weak solutions of carbolic acid.

Two drawbacks to the use of this antiseptic are its benumbing effects on the fingers of the surgeon and the cracking and chapping of the skin where the solution is in contact.

Formalin.-This is a 40 per cent. solution of formaldehyd in water, and is used for disinfecting wounds and instruments and for irrigating purposes. For the former use 2 per cent. solutions and for the latter 0.25 per cent. solutions. It is irritating not only to the wounded areas, but to the eyes and nose of the patient.

Hydrogen Peroxid.-How much germicidal power this drug possesses is an open question. Nevertheless it is particularly useful in cleaning wounds and cavities contaminated with pus and necrotic tissue. When hydrogen peroxid comes in contact with dead organic material it sets oxygen free and destroys the pus or necrotic tissue.

When injected into deep pockets or cavities there 
must be free exit for the foaming material, otherwise the tissues are "ballooned up" and injury may result.

Iodoform.-This substance is said to be devoid of power to inhibit bacterial growth, but does overcome the noxious action of the products of decomposition and fermentation. It is used especially in the treatment of ulcers and abscesses of tubercular origin.

This drug is also capable of producing toxic symptoms through absorption. The most common signs are headache, nausea, loss of appetite, skin eruption, and mental exhilaration.

Lead-and-alum Lotion.-This is much used as a moist dressing, both for its astringent and antiseptic action. It is prepared by mixing together equal parts of two solutions, one of which is alum, 6 drams; water, I pint; the other is lead acetate, 9 drams, and 30 grains in I pint of water.

Boric Acid.-This is an efficient antiseptic of the weak class, and is used extensively in eye, ear, nose, mouth, and bladder antisepsis. It is employed as a saturated solution ( $1: 25$ or weaker).

Silver Salts. - Silver nitrate and the recent organic silver combinations, as protargol, argonin, argyrol, collargol, nargol, etc., are much used in the treatment of purulent conditions due especially to the gonococcus.

Creolin.-This substance is an efficient non-irritating and non-toxic antiseptic. It is used for irrigating and for vaginal antisepsis. With water it forms a soapy emulsion. Used in I-4 per cent. solutions.

Lysol.-Same action and uses as Creolin.

Potassium Permanganate.-This antiseptic is used in disinfecting the hands before operations, for irriga- 
ting purposes, and as a dressing, especially for foulsmelling wounds and ulcers.

After scrubbing the hands well with soap and water, they are immersed in a saturated solution of potassium permanganate, then dccolorized with a saturated solution of oxalic acid, and rinsed in sterile water.

Aristol.-This is a compound of iodin and thymol, and is used as a substitute for iodoform. It is nonirritating, non-toxic, and devoid of odor. Useful as a dusting-powder for ulcers.

Resorcin.-This substance is allied to carbolic acid. It is employed as an application in cellulitis and erysipelas. Also useful in washing out the bladder (3 per cent. solution).

Ichthyol.-This is a very valuable mild and nonirritating antiseptic, used extensively in erysipelas, gonococcal infections, abscesses, and cellulitis. It may be used pure or in watery solutions of $\mathrm{I}: 20$ to $\mathrm{I}: 2$.

Borax.-This is used for the same purposes as Boric Acid.

Turpentine.-This oil is a very useful antiseptic for the disinfection of ragged, lacerated wounds resulting especially from car, wagon, and machinery accidents.

\section{DISINFECTION}

We will consider the disinfection of the sick room, bedclothing, wearing apparel, and the excreta of the patient.

The room of a person ill with a contagious disease should always be disinfected after the patient becomes well. For this purpose we possess several good disin- 
fectants, as sulfur, formaldehyd, and bichlorid of mercury.

Sulfur Fumigation.-The room is closed as tightly as possible; the crevices of the windows and doors are filled by packing them with old newspapers. The bedclothing and the wearing apparel used by the patient and nurse are hung about the room loosely. Powdered sulfur is placed in a metal vessel, which in turn is placed in a broad shallow tin or zinc basin to prevent fire. The sulfur is lighted by placing on it a small shovelful of glowing coals or by mixing with the sulfur a small quantity of alcohol and lighting it with a match. Then leave the room and close the door tightly. The room is to remain closed for twenty-four hours, when it is well ventilated by opening all of the windows. Two pounds of sulfur are sufficient for every IOoO cubic feet of space to be disinfected.

Formaldehyd Fumigation.-This method not only furnishes the best results, but it is absolute. The gas penetrates deeply, does not destroy the most delicate fabrics, will not bleach, and is easily manipulated.

The room, as for sulfur fumigation, must be absolutely tight. The clothing should be hung loosely about the room; all drawers of the bureaus and chiffoniers opened, and books standing on their edges with the leaves separated. The ca. pets and clothing should be sprinkled with water.

Formaldehyd gas may be set free in several ways: By heating wood alcohol; by heating solid formaldehyd; by heating formalin; by treating formalin with chemicals. This last means is very simple, handy, and efficient. The formalin may be treated with sulphuric 
acid and unslaked lime or by potassium permanganate. To 6 ounces of formalin add 2 ounces of sulphuric acid and I pound of unslaked lime, or add to I pint of formalin $6 \frac{1}{2}$ ounces of potassium permanganate. Either of these two methods will set sufficient gas free for IO0O cubic feet of space and requires no special apparatus.

The room is closed for twenty-four hours and then thoroughly ventilated.

Bichlorid of Mercury Method.-By this method the floor, walls, furniture, and wood work are thoroughly scrubbed with soap and hot water and then wiped with a cloth moistened with a I : 500 solution of bichlorid of mercury. The room is then ventilated well for a day or more.

Bedclothing.-Before the clothing from the beds used by persons affected by a contagious or infectious disease are sent to the laundry they should be soaked for twelve hours in a carbolic acid solution ( 1 : IO) or a bichlorid of mercury solution ( $1: 500)$. After this they may be boiled, washed, and hung up in the open air for a day or more.

\section{EXCRETA DISINFECTION}

Sputum.-The sputum of patients ill with pulmonary tuberculosis, pneumonia, and influenza is highly infectious, and should be collected and destroyed. Cups may be used for collecting the sputum. Some advise placing a strong antiseptic solution in the cup, which does not seem safe, but if cups are to be used fill them partially with water and add the antiseptic before the cups are emptied. A better way to dispose 
of the sputum is to have the patient expectorate into a piece of clean soft cloth and burn it immediately.

Urine and Feces.-In cases of typhoid fever these excrements are swarming with germs and should be rendered innocuous.

The disinfectant should not only be added to the excretions, but should be placed in the urinal or bedpan before they are used. Among the best disinfectants for this purpose are carbolic acid (I : IO), bichlorid of mercury ( $\mathrm{I}: 500$ ), chlorinated lime (full strength), copper sulphate or blue vitriol (I :4), iron sulphate or copperas (I:4), zinc sulphate (I : IO). For obvious reasons the chlorinated lime should not be placed in the vessel before the patient uses it, and the bichlorid solution should not be placed in metallic dishes. The mixture of excrement with the disinfectant should not be emptied at once, but allowed to stand several hours.

\section{TOPICAL MEDICATION}

\section{COUNTER-IRRITATION}

When certain drugs are applied to the cutaneous surface a reddening of the skin is produced by stimulating the peripheral circulation and attracting blood from distant parts. This is known as counter-irritation.

In inflammation the first phenomena to occur are increase of blood to the part and transudation of serum and cells from the blood-vessels. If an increased amount of blood can be attracted to other parts of the body, then to a great extent the stasis of blood and transudation in the inflammatory areas may be lessened. It is for this reason that counter-irritation is employed. 
Agents.-Among the more common counter-irritants are: Ammonia, capsicum, iodin, mustard, turpentine oil (rubefacients); acetic acid, cantharides, ammonia, kerosene, and croton oil (vesicants).

Indications.-To relieve pain ; to aid in the absorption of fluid accumulations; to overcome inflammatory processes. Counter-irritation is useful in bronchitis, pleurisy, pneumonia, vomiting, convulsions, abdominal colic, diarrhea, rheumatism, neuralgias, meningitis, coma, apoplexy, effusions of the pleura, pericardium, and peritoneum.

Rubefacients produce redness of the skin, and if the action is prolonged blisters result. The vesicants cause blister formation.

Mustard.-This is generally applied as a plaster, made by mixing powdered mustard and flour in a proportion of $I: I$ to $I: 8$, depending on the patient, the condition of the skin, and the site of application. The mixture of the mustard and flour is moistened with warm vinegar or water and stirred until it becomes of the desired consistence, when the paste is spread on thin cloth and applied to the part. The mustard paste is also prepared by adding the mustard to a regular flaxseed paste.

Mustard-plasters are left on the skin until the surface is reddened or pain results, when the plaster should be removed and a new one applied over another area. Mustard-plasters generally act sufficiently in fifteen to thirty minutes. If the red area resulting from the use of a mustard-plaster is very painful or irritating, it may be dressed with vaselin or bismuth subnitrate. 
Mustard may also be applied as a liniment or cerate.

Capsicum.-This is used mostly in combination with other spices, in what is known as the spice-poultice. This is prepared by mixing (in powdered form) ginger, cloves, cinnamon, and allspice, of each equal parts, and one-half part of Cayenne pepper or capsicum. These are made into a small pillow or bag of cloth and moistened with warm vinegar when used.

Turpentine Oil.- This is best applied as a mixture of warm lard or cotton-seed oil and turpentine, in the proportion of 6 parts of the former to $\mathrm{I}$ of the latter.

Ammonia.-A most efficient way of applying ammonia as a counter-irritant is as a liniment, composed of equal parts of ammonia-water, alcohol, and soap liniment.

A drop or two of strong ammonia-water is applied to the skin and kept free from air by placing over it an inverted watch-crystal or small glass vial (to blister).

Cantharides (Spanish Fly) is extensively used as a vesicant.

Blistering is employed to aid in the absorption of pleural, pericardial, articular, and meningeal effusions. Blistering agents act by dilating the peripheral vessels, which relieves some tension in the area of effusion, and by dilating the lymphatics, which absorb the effused fluid.

Cantharides may be applied as a plaster, cerate, liniment, or collodion. The plaster is either cut in narrow strips and applied so as to intersect and cross each other or it may be cut into small squares and applied. .Do not cover a very large area, as poisoning may follow absorption. 
It takes from four to twelve hours to raise a blister. When the plaster is removed apply an oily or bismuth dressing. To open a blister, do not puncture the raised epithelia, but enter the point of a curved needle in the skin near the base of the blister and force it up under the center of the blister, so as to leave a complete epithelial covering on the area.

Kerosene Oil.-This is applied with equal parts of olive or cotton-seed oils.

Selection of Site.-For analgesic effect apply the medicament over the seat of pain, unless this is referred when the application is made at the origin of the pain: in the knee pain of hip-joint disease the counter-irritation should be made over the hip and not the knee. In neuralgias and neuritis place a narrow strip of plaster along the course of the nerve. To overcome inflammatory processes the application should be made at some distance from the seat of trouble. In meningitis, cerebral congestion, apoplexy, and coma apply a vesicant at the nape of the neck and a mustard-plaster to the abdomen and legs. In pleurisy, bronchitis, and asthma apply the counter-irritant to the abdomen and over the seat of pain. To influence fluid accumulations, as in pleurisy with effusion, the application is made below the nipple and in the axillary line; in pericarditis with effusion, at the base of the heart; in peritonitis, over the tender area. For vomiting apply a mustardplaster over the epigastrium or "pit of the stomach."

Contraindications to the employment of vesicants are lack of vitality, such as results from long-continued fevers, extremes of age, infancy and old age, and paralytic conditions. Obstinate ulcers may result. 


\section{POULTICES}

Poultices are used for the purpose of applying moisture with heat to relieve tension and pain and to relax the blood-vessels. They are employed in the first stage of inflammatory processes and should not be used when the inflammatory area is denuded, as the formation of pus is then enhanced and the extension of the process augmented.

In preparing poultices two points are to be borne in mind: The material of which the poultice is to be made is stirred into the hot water and not the hot water stirred into the material; second, the flannel upon which you smear the mass should be hot. The poultice should at least be an inch thick. Do not allow a poultice to remain on the patient until it is cold, but change early.

Flaxseed Poultice.-Into hot water rapidly stir flaxseed meal, which is gradually poured upon the hot water until it is of the consistence of porridge, then spread thickly on hot woolen cloth, apply, and cover with oiled muslin.

Bread Poultice.-This is used especially for eye affections and is prepared in two ways: Take a thick slice of dry bread and pour hot water upon it, after a minute or so drain off the excess of water and pour on more of the hot liquid, then break the bread and apply; or, allow the bread to simmer in hot water for ten minutes, then mash it into a pulp and apply.

Charcoal Poultice.-This poultice is employed to free putrid ulcers of their odor. To the flaxseed or bread poultice add powdered charcoal. 


\section{STUPES}

Probably the most-employed stupe is that made with turpentine.

Turpentine Stupe.-This is used especially in the relief of intestinal distention of typhoid fever, pneumonia, and intestinal disorders. It may be prepared in two ways: A piece of flannel is soaked in very hot water and then wrung nearly dry, when turpentine (20 drops) is sprinkled upon it; or, 30 to 60 drops of turpentine are added to hot water and a piece of flannel is immersed in the solution. The oil which floats on the water will be taken up by the cloth. Wring nearly dry and apply.

Chloroform-and-turpentine Stupe.-If after the turpentine stupe is prepared a few drops of chloroform are sprinkled upon it an excellent stupe results.

Stupes should be frequently changed. Care should be taken that turpentine-poisoning does not result from absorption of the drug. This may be prevented by alternating the turpentine stupe with one of plain hot water.

\section{CUPPING}

Cupping is a form of counter-irritation used much more in the past than at present. By it blood is drawn to the surface from the deeper parts. Cupping may be performed with wet or dry cups.

Dry Cupping.- The cups used expressly for this purpose have at their summit an air plug, to which may be attached an exhaust pump. The cup is applied to the skin somewhat firmly and by means of the pump the air is withdrawn from the cup, and, in order to equalize the pressure, this loss of air is replaced by 
a swelling of the skin and tissues into the cup, due to an accumulation of blood in the parts. The cups are allowed to remain in place until they fall off,.when they are applied to another part.

The special apparatus described above is not necessary, common graduated medicine-glasses or wineglasses will accomplish the work fully as well. The inside of the glass is moistened with alcohol, the excess of alcohol is removed, and a lighted match applied to the inside of the glass. The alcohol will immediately take fire. When the alcohol is consumed the cup is firmly applied to the skin and will soon take hold. Allow the cup to remain in place until it releases itself.

Dry cupping is used in congestion and edema of the lungs and in kidney disease.

Wet Cupping.-This is performed exactly the same as dry cupping, except that the area of skin to be cupped is first scarified and the cup then applied as above. By this means the blood is not only drawn to the surface, but into the cups, thus depleting the tissues. After completing the work the cupped areas should be dressed as an aseptic wound.

Leeching.-See under Hirudo (Part II.).

\section{APPLICATION OF HEAT}

Varieties.-Heat is applied in two forms : Dry and moist.

Indications.-To relieve pain ; to overcome spasms ; to stimulate the functions of the skin; to counteract inflammatory processes; to equalize circulation; to preserve the vitality of parts.

To Relieve Pain.-For this purpose nothing is more 
efficient than the application of heat, unless it be cold (in selected cases). Heat is particularly useful in the pain of pleurisy (hot-water bag or poultice), of sprains (prolonged immersion in hot water), of eye diseases (fomentations), of ear troubles (douching and prolonged irrigation), of dysmenorrhea (sitz-bath).

To overcome spasms of muscles in cases of fracture, sprains, lumbago, wry neck, croup, spasmodic dysmenorrhea, convulsions (infantile, uremic, and puerperal), chordee, and cramps of the legs.

To stimulate the functions of the skin (sweating) in nephritis, uremia, and eclampsia.

To counteract inflammatory processes in rheumatism and, in fact, all superficial inflammations.

To equalize the circulation in shock, collapse, and chills.

To preserve the vitality of tissues in injuries of all kinds when life of the part is in danger.

\section{FORMS OF APPLICATION}

Poultices.-See under Topical Medication.

Hot-water Bag.-This is one of the most-employed and handiest forms of applying heat. The bag should not be so full that it is weighty and cumbersome, especially when used on the abdomen or chest. When hot-water bags are used on unconscious and comatose persons care should be taken that burns do not result, as the skin of these patients burn easily because of its lowered vitality and on account of the patient's inability to realize the amount of heat applied. It is always well to have a piece of woolen cloth between the bag and the skin. 
Good substitutes for hot-water bags are found in hotwater bottles, which are large glass bottles (fully tested) filled with hot water, and hot bricks or hot flat-irons.

Hot fomentations are simply woolen cloths wrung out in hot water.

Hot-water baths are useful in infantile convulsions, nephritis, and uremia. The water should be about $104^{\circ} \mathrm{F}$. and the room should be warm.

Hot packs are similar to the cold packs, except that hot water is employed instead of cold water. The full hot pack is especially useful in uremia and eclampsia. A woolen blanket is immersed in hot water and then run through a clothes-wringer until nearly dry, when it is wrapped about the patient.

Sitz-bath.-This consists of an immersion of the hips and buttocks in hot water and steam vapor. It is useful in dysmenorrhea, suppressed menstruation, and pelvic disorders.

Hot-air Baths.- The best form of hot-air bath for the patient who can walk about is the Turkish bath, which consists of a series of rooms, each successive room having a higher temperature, which greatly increases the output of sweat. The final step is a rubbing and shampooing of the whole body, followed by a cold-water plunge or shower. After completion of the bath a rest is necessary before going into the outer air. For bed patients who need hot-air applications the bed-bath is employed, which consists of covering the patient (excepting the head) with blankets and introducing under the cover hot air conducted by a tin pipe from a near-by lamp. Another form of hot-air bath is the cabinet-bath, which is used for patients not confined 
to the bed. The patient is seated on a stool and is totally enveloped, excepting the head, with a special cabinet constructed for the purpose or with a large blanket. Heat is produced by a lamp placed under the chair of the patient or, better, outside of the apparatus and conducted within by means of a pipe. The baking machine is the last form of hot-air bathing to be considered. It is especially useful in the treatment of chronic joint affections. The limb is suspended in the metal compartment of the apparatus, care being taken that the limb does not touch the machine, as a burn will be produced. The air in the machine is raised to a temperature of $200^{\circ}$ to $300^{\circ} \mathrm{F}$. by means of a lamp placed beneath the machine. The treatment should continue about one hour.

In all forms of hot-air bathing if sweating does not begin it may be started by giving the patient a drink of cold water.

Russian Bath.-This consists of applying moist heat to the body in the form of heated vapors.

\section{RECTAL ALIMENTATION AND MEDICATION}

Medicaments are administered by the rectum in two forms : Enemata and suppositories.

The indications for the use of enemata are: To provide nourishment, to empty the lower part of the bowel, to administer drugs for general action, for local action, and to supply the body with fluid.

To Provide Nourishment.-We use for this purpose what are known as nutritive encmata. They are composed of concentrated, digested, and easily assimilable material. Food is given by rectum only when it is 
impossible to give it by mouth, as in cases of excessive and persistent vomiting, in unconscious, comatose, delirious, and refractory patients, when there is grave disease of the stomach, as ulcer and cancer, in pharyngeal paralysis, etc.

It has been repeatedly shown that proteid in solution, egg-albumin, saline material, and water are capable of being absorbed in the lower colon or rectum.

A very good nutritive enema is prepared by mixing peptonized milk, 4 ounces; liquid beef preparation, $\frac{1}{2}$ ounce; white of I egg, and about 20 grains of salt. To this a small quantity of whisky may be added.

The rectum should first be emptied with a small enema of plain water about one-half hour before the nutritive enema is given.

To Empty the Bowel.-There are several forms of enemata employed to empty the lower bowel :

Soapsuds Enema.-This is one of the simple enemata, and is prepared by mixing thoroughly $\frac{1}{2}$ ounce of soft soap with I pint of warm water. It is also made by agitating a bar of common soap in warm water until it is quite soapy.

Simple Mixed Enema.-Add to the soapsuds enema $\frac{1}{2}$ ounce of molasses and I dram of salt.

Turpentine Enema.-To the soapsuds enema add $\frac{1}{2}$ ounce or less of oil of turpentine. This is very useful in relieving tympanites.

Asafitida Enema.-To I 2 ounces of warm water add 4 ounces of asafetida emulsion (prepared by agitating $\frac{1}{2}$ dram of asafetida powder in 4 ounces of water). This is useful in tympanites and infantile colic.

Glycerin Enema.-To I ounce of warm water add I 
ounce of glycerin. It is best given with a small hardrubber syringe.

Oil Enema.-Use I pint of warm cotton-seed oil.

Purgative Encma.-This is a strong-acting enema and is employed when the simple enemata fail. It is given high, through a rectal tube. It consists of soapsuds solution, I pint; glycerin, I ounce; magnesium sulphate, $\frac{1}{2}$ ounce; oil of turpentine, $\frac{1}{2}$ ounce.

Ox-gall Encma.-To the purgative enema add i 5 grains of powdered ox-gall.

For General Medication.-If for any reason medicines cannot be administered by mouth, as in persistent vomiting, unconsciousness or deliriousness of the patient, or on account of ill-taste of medicines, they may be given by rectum. Any drug except those of a corrosive character may be administered in this way. Give them in as small bulk as possible to prevent their expulsion.

In diarrhea the starch-and-laudanum enema is much employed. Make a thin starch paste in the usual way (hot method), and to 2 ounces of this paste add the laudanum ( 5 to 20 drops, depending on the age and condition of the patient). This enema is best given by means of a small hand syringe.

For Local Medication.-In diseases of the rectum and neighboring organs medication is often applied by the rectum. We will consider but one-the quassia enema-which is used in treating pin-worms of the rectum. To I dram of quassia chips add 8 ounces of cold water, allow to stand two or three hours, strain, and use as a single injection.

To Supply Fluid.-See Enteroclysis. 


\section{ANTITOXINS AND SERUMS}

Space will not be given to the discussion of the formation and production of antitoxins.

Diphtheria Antitoxin.--Since this serum has come into practical use the mortality from diphtheria has greatly decreased; the disease runs a shorter and less severe course in many instances and the complications are less frequent.

Administration.-Antitoxin is to be given as soon as diphtheria is suspected. The initial dose in cases of moderate severity should be at least 3000 units. After giving antitoxin the symptoms soon begin to lessen, the patient feels easier, the pulse is better, the temperature decreases, and the local patches in the mouth and throat look less angry and begin to grow smaller. If improvement does not begin within twelve hours the dose of antitoxin should be repeated. In severe cases the serum should be given at regular intervals.

Antitoxin must be given early in the disease and in sufficient quantity. The danger is not in overdosing, but in underdosing.

Ill Effects of Antitoxin.-After the administration of antitoxin certain symptoms may arise which are due not to the antitoxin, but probably to the horse serum which contains the antitoxin. These symptoms are, as a rule, trivial, but may cause alarm if their meaning is not understood. The principal after-effects are: Scarlatiniform rash, urticaria, pain, tenderness, and swelling of the joints. These symptoms may occur within a few minutes after giving the antitoxin or may be delayed for several days or weeks. 
Immunizing Effect.-In small doses (500 units) the antitoxin will act as a prophylactic against diphtheria in persons exposed to the disease. Nurses attending diphtheria patients and all persons exposed to the disease should receive an immunizing dose of antitoxin.

Site of Injection.-The subcutaneous injection of antitoxin may be given at any suitable site, but probably the best place is between the scapulæ, as the patient cannot witness the manipulations and the protection of this area is good.

Before giving the injection the area of skin should be cleansed as in performing a hypodermoclysis.

Antistreptococcus Serum.-The discovery of this serum is due to the efforts of Marmorek (1895).

This serum is employed in the treatment of disease supposed to be caused by the streptococci. The results of its use vary, but in many instances have been most happy.

It is employed in erysipelas, scarlet fever, puerperal sepsis, pyemia, septicemia, and acute articular rheumatism.

Dosage.-This serum is administered in the same way and manner as diphtheria antitoxin. The initial dose is Io cc., and may be repeated.

Tetanus Antitoxin.-This serum has been used most successfully to prevent tetanus, but after the disease is established it is doubtful if the antitoxin is of great value.

The antitoxin should be given as a prophylactic to patients suffering from wounds produced by rusty nails, slivers of old wood, blank cartridges, fireworks, and accidents occurring in barns or when filth of the 
street is incorporated in the wound. It is well known that the tetanus bacilli are found around barns and in the roads where excreta from animals abound.

Dosage.-This serum must be given early and in frequently repeated doses of $30 \mathrm{cc}$. each.

Antipneumococcus Serum.-The use of this serum is still in its infancy. Good results have been reported after its employment. It is said to be abortive and that it lessens the severity of the disease and causes the fever to fall by lysis instead of crisis.

Dosage.-Of this serum I $5 \mathrm{cc}$. should be given every four to six hours during the height of the disease.

Cholera Antitoxin.-An antitoxin against cholera has been found, but is said to be more of a prophylactic than curative.

Antidysenteric Serum.-This serum has lately passed from the experimental to the practical stage. It is used in dysentery due to Shiga's bacillus. Its usefulness depends on early and sufficient administration.

Dosage.-Of this serum ro cc. are given two to four times a day during the early stages of the disease.

Other antitoxins are constantly being experimented with and made practical. They are those against yellow fever, typhoid ferer, bubonic plague, tuberculosis, syphilis, rabies, cancer, and snake venom. 


\section{PART VI}

\section{ADDEndA}

\section{STRENGTH OF DRUG PREPARATIONS}

Cerates.

Camphor,

Cantharides,

Lead Subacetate,

Resin,

Simple,

Spermaceti,

Decoctions.

Cetraria,

Sarsaparilla Compound,

\section{Emulsions.}

Almond,

Ammoniac,

Asafetida,

Chloroform,

Extracts.

Aloes,

Belladonna,

Cannabis Indica,

Cascara,

Cimicifuga,

Colchicum Root,

Colocynth,

Digitalis,

Ergot,
PER CENT.

\section{Extracts.}

$\begin{array}{rlr}2 & \text { Euonymus, } & 400 \\ 32 & \text { Gentian, } & 250 \\ 20 & \text { Hematoxylon, } & -\end{array}$

35

- Jalap

2 Krameria,

Leptandra,

Licorice,

Malt,

Nux Vomica,

Opium,

Physostigma,

Quassia,

Rhubarb,

Scopola,

Stramonium,

(Resin), 60

$0.3^{1}$

PBR CENT.

5

6

4

4

4

Sumbul,

800

400

Taraxacum,

Fluidextracts.

$800 \quad$ All fluidextracts are of the

I. $4^{1}$ same strength-i.e., I

$600 \quad \operatorname{minim}=1$ grain of the

drug-except the follow-

500 ing :

1 Percentage of principal alkaloid. 
Fluidextracts.

PER CENT

Aconite,
Belladonna Root,
Cinchona,
Coca,
Colchicum Seed,
Conium,
Gelsemium,
Hydrastis,
Hyoscyamus,
Ipecac,
Nux Vomica,
Pilocarpus,
Scopola,
Stramonium,

Glycerites.

Boroglycerid,

Carbolic Acid,

31

Hydrastis,

Starch,

Tannic Acid,

Yelk of Egg,

Honey.

Rose,

Infusions.

Cinchona,

Digitalis,

Senna Compound,

Wild Cherry,

$0.5^{1}$

$0.5^{1}$

$4^{1}$

$0.5^{1}$

$0.5^{1}$

$0.5^{1}$

$0.3^{1}$

$2^{1}$

$0.075^{1}$

$1.75^{1}$

$I^{1}$

$0.4^{1}$

$0.5^{1}$

$0.35^{1}$

Liniments.

PER CENT.

$\begin{array}{lc}\text { Soap, } & 6 \\ \text { Soft, } & 65 \\ \text { Turpentine, } & 35 \\ \text { Masses. } & \\ \text { Copaiba, } & 94 \\ \text { Iron Carbonate-Vallet's, } & 50 \\ \text { Mercury, } & 33 \\ \text { Mixtures. } & \\ \text { Chalk, } & 20 \\ \text { Iron Compound (Sulphate) } & \text { 0.7 } \\ \text { Licorice Compound, } & - \\ \text { Rhubarb and Soda, } & \mathbf{1 . 5}\end{array}$

Mucilages.

Acacia,

Elm,

Sassafras,

Tragacanth,

10 Ointments.

20

Belladonna,

IO

Carbolic Acid,

Chrysarobin,

12

Diachylon,

Iodin,

Iodoform,

Liniments.

$\begin{array}{lr}\text { Ammoniac, } & 35 \\ \text { Belladonna, } & 95 \\ \text { Camphor, } & 2 \\ \text { Chloroform, } & 30 \\ \text { Lime, } & 50 \\ \text { Mustard Compound, } & 3\end{array}$

Lead Carbonate,

Nitrate, Oxid, Red, Io Yellow, Io

Nutgall, 20

Potassium Iodid, Io

Rose-water (Cold Cream), -

Stramonium,

10

${ }^{1}$ Percentage of principal alkaloid. 
Ointments.

Sulphur,

Veratrin,

Zinc Oxid,

Oleates.

Atropin,

Cocain,

Mercury,

Quinin,

Veratrin,

Zinc,

Plasters.

Ammoniac and Mercury, 30

Arnica,

Belladonna,

Capsicum,

Iron,

Isinglass,

Lead,

Mercury,

Opium,

Pitch, Burgundy, Cantharidal,

Resin,

Soap,

Solutions.

Ammonium Acetate,

Arsenous Acid,

Arsen. and Merc. Iodids, I

Iodin Compound,

Iron Acetate,

and Ammonium Acetate, $4^{3}$

Chlorid,

Citrate,

Indefinite.

9
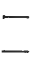

30

6

80

8

14

IO
PER CENT.

Solutions.

Iron Nitrate,

Subsulphate,

Tersulphate,

Lead Subacetate,

Dilute,

Lime,

Mercury Nitrate,

Potassium Arsenite,

Citrate,

Hydrate,

Sodium Arsenate,

Hydrate,

Hypochlorite (Chlorin), 2.6

Zinc Chlorid,

$0.4^{1} \quad$ Spirits.

ite.

$\begin{array}{lc}\text { Almond, Bitter, } & \text { I } \\ \text { Ammonia, } & \text { Io } \\ \text { Aromatic, } & 3.4^{4} \\ \text { Anise, } & \text { Io } \\ \text { Camphor, } & \text { Io } \\ \text { Chloroform, } & 6 \\ \text { Cinnamon, } & \text { Io } \\ \text { Ether, } & 3^{2.5} \\ \text { Compound, } & 32.5 \\ \text { Nitrous, } & 4^{5} \\ \text { Frumenti (Whisky), } & 37-47.5^{6} \\ \text { Gaultheria, } & 5 \\ \text { Juniper, } & 5 \\ \text { Compound, } & 0.8 \\ \text { Lavender, } & 5 \\ \text { Lemon, } & 5 \\ \text { Nitroglycerin, } & \text { I } \\ \text { Nutmeg, } & 5 \\ \text { Peppermint, } & \text { Io }\end{array}$

1 Percentage of principal alkaloid.

${ }^{2}$ Percentage of metallic iron.

3 Percentage of tincture of iron chlorid.

4 Percentage of ammonium carbonate.

${ }^{5}$ Percentage of ethyl nitrite.

${ }^{6}$ Percentage of absolute alcohol. 
Spirits.

Phosphorus,

Spearmint,

\section{Syrups.}

Acacia,

Almonds,

Blackberry,

Citric Acid,

Garlic,

Ginger,

Hydriodic Acid,

Ipecac,

Iron Iodid,

Krameria,

Lactucarium,

Lime,

Marshmallow,

Orange,

Rhubarb,

Rose,

Sarsaparilla Compound,

Senega,

Squill,

Tar,

Tolu,

Wild Cherry,

Tinctures.

All tinctures are of 20 per cent. strength except the following:

Aconite,

Aloes, and Myrrh,

Arnica Root,

Belladonna Leaves,

Benzoin Compound,

Percentage of absolute alcohol.
Tinctures.

o.12 Bryonia, Io

Io Cannabis Indica, Io

Cantharides, 10

Capsicum, 10

Chirata, Io

Colchicum Seed, Io

Digitalis, Io

Gelsemium, 10

Gentian Compound, Io

Hyoscyamus, Io

Iodin, 7

Hecac and Opium, 10

Iron Chlorid, $\quad \mathbf{1 3 . 6}$

Kino, 5

Lactucarium, $\quad 50$

Lemon, $\quad 50$

Lobelia, IO

Matico, Io

Musk, $\quad 5$

Nux Vomica, Io

Opium, 10

Camphorated, $\quad 0.4$

Deodorized, Io

$45^{2} \quad$ Physostigma, I0

7.5 Sanguinaria, Io

I Squill, 10

15 Stramonium, 10

Strophanthus, IO

Sumbul, IO

Veratrum Viride, $\quad$ xo

Vinegars.

Opium,

10

Squill,

Io

Waters.

Almond, Bitter, - $\quad$ o.I

Ammonia, Io

Strong, $\quad 28$

"Percentage of vinegar of squill. 
PER CENT.

Waters.

Anise,

Camphor,

Chlorin,

Chloroform,

Cinnamon,

Creosote,

Fennel,

Hydrogen Dioxid,

Orange Flower,

Peppermint,

Rose,

Strong,
Waters.

0.2 Spearmint,

0.8 Wines.

0.4 Antimony,

0.5 Colchicum Root,

0.2 Seed,

Ergot,

0.2 Ipecac,

3 Iron, Bitter,

Citrate,

o.2 Opium,

Red,

Saturate. White,

${ }^{1}$ Percentage of absolute alcohol.
PER CENT.

0.2

0.4

40

IO

20

IO

5

4

IO

$7-12^{1}$

$7-12^{1}$ 


\section{SYNONYMS}

Absinth-Wormwood.

Acetanilid - Antifebrin, phenylacetamid.

Aconite-Wolfsbane, monkshood. Adonis Vernalis-False hellebore. Allium-Garlic.

Allspice-Pimenta.

Althæa-Marshmallow.

Ammonium Chlorid-Sal ammoniac.

Amylum-Starch.

Anthemis-Roman camomile.

Antifebrin-Acetanilid.

Apium-Celery.

Apocynum-Canada hemp.

Arbor Vitæ-Thuja.

Argentum Nitrate-Silver nitrate.

Asclepias-Pleurisy root.

Aspidium-Male fern, Filix mas.

Avena Sativa-Oats.

Baptisia-Wild indigo.

Barosma-Buchu.

Bearberry-Uva Ursi.

Belladonna-Deadly nightshade.

Black Haw-Viburnum Prunifolium.

Snakeroot-Cimicifuga.

Bladder Wrack-Fucus Vesiculosis.

Bloodroot-Sanguinaria.

Blue Flag-Iris.

Vitriol-Copper sulphate.

Boneset-Eupatorium.

Broom-tops-Scoparius.

Buchu-Barosma.

Buckthorn-Rhamnus Frangula.

Burdock-Lappa.

Butter Nut-Juglans.

Cactus-Night-blooming cereus.

Calabar Bean-Physostigma.

Calamus-Sweet flag.

Calendula-Marigold.

Canada Hemp-A pocynum.

Cannabis Indica-Indian hemp.

Cantharides-Spanish fly.

Capsicum-Cayenne or red pepper.

Caraway-Carum.

Carbo Ligni-Charcoal.

Carbolic Acid-Phenol, phenic acid.

Caryophyllus-Clove.

Cascara-Rhamnus Purshiana.

Cassia-Cinnamon.

Castanea-Chestnut.

Castor Oil-Oleum Ricini.

Caulophyllum-Blue Cohosh.

Celery-Apium.

Cereus-Cactus.

Chamomile, German-Matricaria.

Roman-Anthemis.

Charcoal-Carbo ligni.

Chenopodium-American wormseed.

Chestnut-Castanea.

Chimaphila-Pipsissewa.

Chionanthus-Fringe-tree.

Cimicifuga - Black snakeroot, black cohosh.

Cinnamon-Cassia. 
Claviceps-Ergot.

Clove-Caryophyllus.

Cochlearia-Horse-radish.

Cohosh, Black-Cimicifuga.

Blue-Caulophyllum.

Cola-Kola.

Colchicum-Meadow saffron.

Conium-Poison hemlock.

Convallaria-Lilly of the valley.

Corn-silk-Zea Mays.

Couch Grass-Triticum.

Cranesbill-Geranium.

Cream of Tartar-Potassium bitartrate.

Creta-Chalk.

Culver's Root-Leptandra.

Cusso-Kousso.

Dandelion-Taraxacum.

Delphinium - Stavesacre, larkspur.

Digitalis-Fox-glove.

Dock, yellow-Rumex.

Dog grass-Triticum.

Dover's Powder - Powder of ipecac and opium.

Elm-Ulmus.

Epsom Salt - Magnesium sulphate.

Ergot-Claviceps Purpurea.

Erigeron-Canada fleabane.

Eriodictyon-Yerba Santa.

Erythroxylon-Coca.

Euonymus-Wahoo.

Eupatorium-Boneset.

False Hellebore - Adonis Vernalis.

Fels Bovis-Ox-gall.

Filix mas-Aspidium, male fern.
Fleabane, Canada-Erigeron.

Fox-glove-Digitalis.

Fringe-tree-Chionanthus.

Fucus-Bladder or sea wrack.

Garlic-Allium.

Gaultheria-Wintergreen.

Gelsemium-Yellow jasmine.

Geranium-Cranesbill.

Ginger-Zingiber.

Glauber Salt-Sodium sulphate.

Glycyrrhiza-Licorice.

Golden Rod-Solidago.

Seal-Hydrastis.

Green Vitriol-Iron sulphate.

Hematoxylon-Logwood.

Hamamelis-Witch hazel.

Hedeoma-Pennyroyal.

Hellebore, American-Veratrum Viride.

False-Adonis Vernalis.

Green-Veratrum Viride.

Helonias-False unicorn.

Hemlock, Poison-Conium.

Hemp, Canada-Apocynum.

Indian-Cannabis Indica.

Henbane-Hyoscyamus.

Hops-Humulus.

Horse-radish-Cochlearia.

Humulus-Hops.

Hydrastis-Golden seal.

Hydrochloric Acid-Muriatic acid.

Hydrocyanic Acid-Prussic acid.

Hyoscyamus-Henbane.

Indigo, Wild-Baptisia.

Iris-Blue flag.

Jaborandi-Pilocarpus.

Jasmine, Yellow-Gelsemium.

Juglans-Butternut. 
Kola-Cola.

Kousso-Cusso.

Krameria-Rhatany.

Lactose-Milk-sugar.

Lactucarium-Lettuce.

Lady Slipper-Cypripedium.

Lappa-Burdock.

Larkspur-Delphinium, Stavesacre.

Lavender-Lavandula.

Leptandra-Culver's root.

Lettuce-Lactucarium.

Licorice-Glycyrrhiza.

Lily of the Valley-Convallaria.

Logwood-Hematoxylon.

Male Fern-Aspidium, Filix mas.

Mandrake - Podophyllum, May apple.

Marigold-Calendula.

Marshmallow-Althæa.

Matricaria-German camomile.

May Apple-Podophyllum.

Mentha Piperita-Peppermint.

Viridis-Spearmint.

Monkshood-Aconite.

Muriatic Acid - Hydrochloric acid.

Muskroot-Sumbul.

Myristica-Nutmeg.

Night-blooming Cereus-Cactus.

Nightshade, Deadly-Belladonna.

Nutmeg-Myristica.

Oak-Quercus.

Oats-Avena Sativa.

Passiflora-Passion flower.

Pennyroyal-Hedeoma.
Pepo-Pumpkin seeds.

Pepper, Cayenne-Capsicum.

Peppermint-Mentha Piperita.

Peruvian Bark-Cinchona.

Petrolatum-Vaselin.

Phenazone-Antipyrin.

Phenol-Carbolic acid.

Phenylacetamid-Acetanilid.

Physostigma-Calabar bean.

Physostigmin-Eserin.

Phytolacca-Poke root or berry.

Pilocarpus-Jaborandi.

Pimenta-Allspice.

Pink-root-Spigelia.

Pipsissewa-Chimaphila.

Pix Liquida-Tar.

Pleurisy Root-Asclepias.

Podophyllum - Mandrake, May apple.

Poke Root or Berry-Phytolacca.

Prickly Ash-Xanthoxylon.

Prunus Virginiana-Wild cherry.

Prussic Acid-Hydrocyanic acid.

Pumpkin Seeds-Pepo.

Quercus-Oak.

Rhamnus Frangula-Buckthorn.

Purshiana-Cascara.

Rhatany-Krameria.

Rheum-Rhubarb.

Rochelle Salt-Sodium and potassium tartrate.

Rumex-Yellow dock.

Sabina-Savin.

Saffron, Meadow-Colchicum.

Sage-Salvia.

Sal Ammoniac-Ammonium chlorid.

Salix-Willow. 
Salvia-Sage.

Sanguinaria-Blood-root.

Santonica-Levant wormseed.

Sarsaparilla-Smilax.

Savin-Sabina.

Scilla-Squill.

Scoparius-Broom tops.

Sea Wrack-Fucus.

Serpentaria-Virginia snakeroot.

Sinapis-Mụstard.

Smilax-Sarsaparilla.

Snakeroot, Black-Cimicifuga.

Virginia-Serpentaria.

Sodium Borate-Borax.

Solidago-Golden rod.

Spanish Fly-Cantharides.

Spearmint-Mentha Viridis.

Spigelia-Pink-root.

Squill-Scilla.

Starch-Amylum.

Star Grass-True unicorn root.

Stavesacre-Delphinium.

Stramonium-Jamestown weed.

Sumbul-Muskroot.

Sweet Flag-Calamus.

Taraxacum-Dandelion.

Tartar Emetic-Potassium and antimony tartrate.

Tea-Thea.
Thea-Tea.

Thuja-Arbor Vitæ.

Triticum-Dog or couch grass.

Ulmus-Elm.

Unicorn Root, False-Helonias.

True-Star grass.

Uva Ursi-Bearberry.

Veratrum Viride-American or green hellebore.

Viburnum Opulus-Cramp bark.

Prunifolium-Black haw.

Wahoo-Euonymus.

Wild Cherry-Prunus Virginiana.

Willow-Salix.

Wintergreen-Gaultheria.

Witch Hazel-Hamamelis.

Wormseed, American-Chenopodium.

Levant-Santonica.

Wormwood-Absinth.

Xanthoxylon-Prickly ash.

Yellow Jessamine-Gelsemium.

Yerba Santa-Eriodictyon.

Zea Mays-Corn-silk.

Zingiber-Ginger. 


\section{WEIGHTS AND MEASURES}

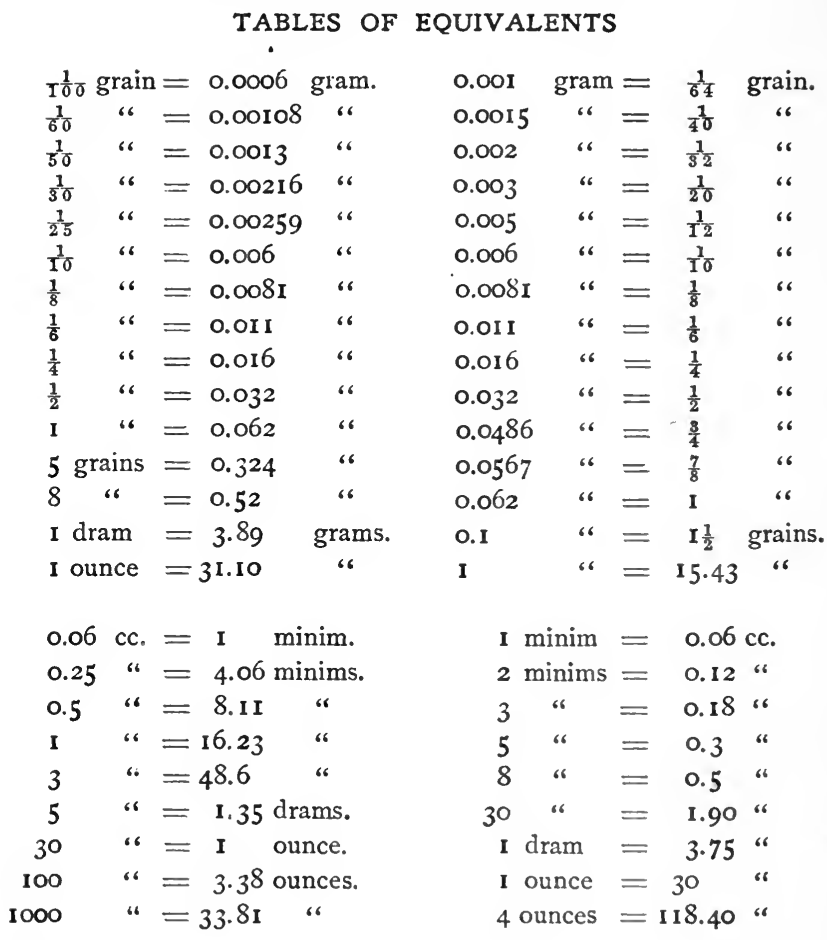

APOTHECARIES' MEASURE

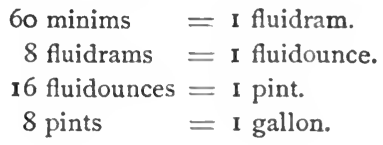




\section{DOMESTIC MEASURES}

(These are only Approxumate)

I teaspoonful = about I dram or 4 cubic centimeters (cc.).

I dessertspoonful $=$ " 2 drams or 8 " " "

I tablespoonful = " $\frac{1}{2}$ ounce or 15 " "

I wineglassful $=$ " 2 ounces or 60 " "

I teacupful " " 5 ounces or 150 " "

15 



\section{N D EX}

Absinth, 165

Absolute alcohol, 46

Acacia, 165

Acetanilid, 40

Acetic acid, 42 glacial, 42

Acetozone, 178

Acetum, I 4, 42

Acid, acetic, 42 dilute, 42 glacial, 42

arsenous, 59

benzoic, 64

boracic, 66, 196

boric, 66 , I96

camphoric, 7 I

carbazotic, I 39

carbolic, 73, 195

cinnamic, 64

citric, 169

gallic, 94

hydrochloric, 103 dilute, I04

hydrocyanic, 105 dilute, 105

muriatic, 103

nitric, 127

nitrohydrochloric, 128

phenic, 73

phosphoric, dilute, I 37

picric, I 39

picronitric, I 39

prussic, I05

salicylic, I 49

sulphuric, 157

aromatic, 157

dilute, I 57

tannic, 94

Acids, administration of, 22

Aconite, 43

preparations, 44

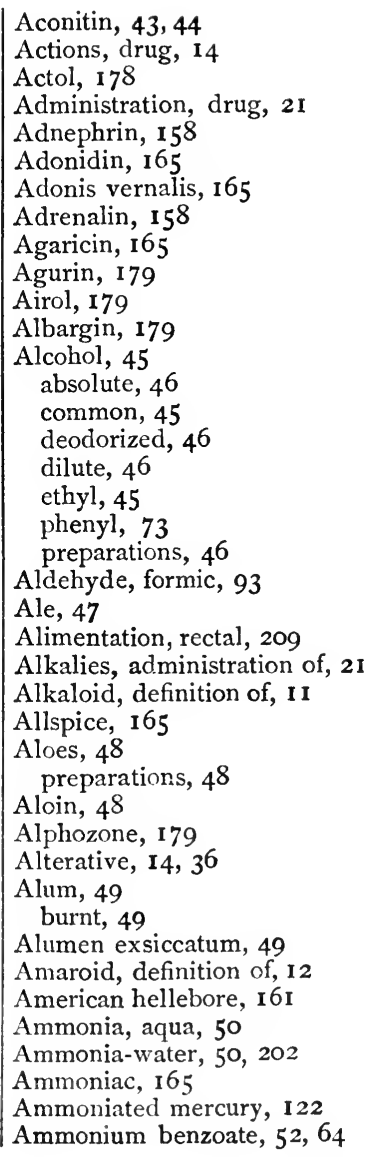


Ammonium bromid, 52 carbonate, 52 chlorid, 53 hypophosphite, 137 iodid, 54 sulpho-ichthyolate, 108 valerianate, 54,161

Amyl nitrite, 54

Amyloform, I79

Analgesic, 14, 33

Anaphrodisiac, 15

Anesthetic, I 5, 32

Anesthol, I79

Animal charcoal, 75

Anise, 165

Anodyne, general, I4, 33 local, 15

Antacid, 15

Anthelmintic, 15, 32

Antidysenteric serum, 214

Anti-emetic, I5, 30

Antifebrin, 40

Antigalactagogue, I6, 38

Antihidrotic, I6, 37

Antimony and potassium tartrate, 55

tartrated, 55

Antiperiodic, I 6

Antipneumococcus serum, 2I 4

Antipyretic, 16, 37

Antipyrin, 57 salicylate, I82

Antiseptic, 16

Antiseptics, 39, 194 intestinal, $3 \mathbf{I}$

urinary, 35

Antisialagogue, 16, 37

Antispasmodic, I7, 33

Antistreptococcus serum, 21 3

Antitoxin, cholera, 2I 4 diphtheria, 2I 2

tetanus, 213

Antitoxins, 212

Aperient, 17

Aphrodisiac, 17

Apiol, I 66

Apium graveolens, 168

Apocynum, 166

Apomorphin, $5^{8}$

Aqua, I4
Aqua hydrogenii peroxidi, I06 regia, 128

Arabic, gum, 165

Arbutin, 176

Argenol, 179

Argentamin, I 79

Argentum nitrate, 152

Argonin, 196

Argyria, 153

Argyrol, 179, 196

Aristochin, 180

Aristol, 180,197

Arnica, 166

Aromatic spirit of ammonia, $5 \mathrm{I}$

Arsenic, administration of, 22

antidote, 6I, II 3

compounds, 59

iodid, $6 \mathrm{r}$

trioxid, 59

white, 59

Arsenous acid, 59

Artificial oil of wintergreen, 96, 149

Asafetida, 166 enema, 210

Aspidium, 166

Aspidosperma, I66

Aspirin, 150

Astringent, 17, 39 intestinal, 32

Atropin, 62 as an antidote, 63

BAG, hot-water, 207 ice-, 188

Balsam of Peru, I 66

Barium chlorid, 166

Basham's mixture, II 2

Bath, bed-, I87. 208

cabinet-, 208

hot-air, 208

hot-water, 208

mercury, I 26

Russian, 209

sitz-, 208

sponge, 185

tub-, I 86

Turkish, 208

Baths, I 85

Bearberry, 176 
Bed-bath, I $\$ 7,208$

Bedclothing, disinfection, 199

Beer, 46

Belladonna, 62 preparations, 62

Benzoic acid, 64

Benzoin compounds, 64

Benzoinated lard, 64

Benzosol, 100, 180

Berberin, I02

Bichlorid of mercury. See under Mercury.

Bismuth benzoate, 65

betanaphtolate, 65

compounds, 65

nitrate, 65

salicylate, 65

subcarbonate, 65

subgallate, 65

subnitrate, 65

Bitter sweet, I 70

Bitters, administration of, $2 \mathbf{I}$

Black cohosh, I68 draught, 176

drop, I 31

haw, 177

snakeroot, 168

wash, 123

Blaud's pills, I I 3

Blistering, 202

Blood root, 175

Blue cohosh, 166

flag, 172

mass, 124

methylene-, I73

ointment, 124

Bone charcoal, 75

Boneset, 167

Boracic acid, 66, 196

Borax, 66, 197

Boric acid, 66, 196

Boroglycerid, 66

Brandy, 46

Bread poultice, 204

Bromism, I 44

Bromoform, I80

Broom tops, I 5 I

Brown mixture, 99

Brucin, 129

Buchu, 167
Buckthorn, I 7 I

Burnt alum, 49

Butyl chloral hydrate, I 70

CABINET-BATH, 208

Cactus, 167

Caffein, 67 citrated, 67

Calabar bean, 138

Calamine, 162

Calamus, I 67

Calcium bromid, 68

carbonate, 68

chlorid, 69

compounds, 68

glycerinophosphate, I37

hypochlorite, 70

hypophosphite, I 37

oxid, 69

phosphate, 138

sulphid, 70

Calisaya, 8o

Calomel, 123

Calumba, 167

Calx, 69 chlorata, 70

sulphurata, 70

Camphor, 70 monobromate, 7 I

Camphorated oil, $7 \mathbf{I}$

Camphoric acid, $7 \mathbf{I}$

Canada hemp, 166

Cannabin, 72 tannate, 72

Cannabis indica, 72

Cantharides, 167,202

Capsicum, 73, 202

Carbazotic acid, 139

Carbo animalis, 75 ligni, 75

Carbolic acid, 73, 195

Carbon, 75

Cardamom, 167

Cardiac sedative, 17,28

stimulant, 17,28

Carminative, 17

Carron oil, 70

Caryophyllus, 169

Cascara sagrada, 75

Cascarin, 75 
Cassia, 168

Castor oil, 76

Cataphoresis, 25

Cataplasm,

Catechu, 168

Cathartic, I8, 30

Caulophyllum, 166

Caustic, 39

lunar, 152

soda, 154

Cayenne pepper, 73

Celery, I 68

Cephaëlin, ro

Cerate, 12, 215

camphor, $7 \mathbf{I}$

cantharides, 167

Goulard's, I 16

lead subacetate, I 16

Cereus grandiflorus, 167

night-blooming, 167

Cerium oxalate, 168

Chalk, 68

Chalybeate, 18

Chamomile, German, 167

Champagne, 46

Charcoal, animal, 75

bone, 75

poultice, 204

vegetable, 75

Chloral, croton, I 70

hydrate, 77 butyl, 170

Chloralamid, 168

Chloralose, 168

Chloretone, I 80

Chlorinated lime, 70

Chloroform, 78

Cholagogue, I8, 3 I

Cholera antitoxin, 214

Chrysarobin, I68

Cimicifuga, I68

Cinchona, 80

Cinchonidin, 80

Cinchonin, 80

Cinchonism, 82

Cinnamic acid, 64

Cinnamon, 168

Citric acid, I69

Classification, drug, 26

Cloves, 169
Coca, 82

Cocain, 82

Cocculus, 169

Codein, I3O, I $3 \mathbf{I}$

Cod-liver oil, 83

Coffee, 67

Cohosh, black, 168

blue, I 66

Cola, 173

Colchicin, 84

Colchicum, 84

Cold, local use of, 185 packs, 187

Collargol, ı80, 196

Collodion, styptic, 94

Colocynth, 169

Colocynthin, 169

Columbo, 167

Coniin, 169

Conium, 169

Constituents, drug, I I

Convallamarin, 86

Convallaria, 86

Copaiba, I69

Copper arsenite, I 69 compounds, 169

sulphate, 169

Copperas, I I 3

Corn-silk, 169

Cornutin, 91

Corrosive sublimate. See Mercury bichlorid.

Cotarnin hydrochlorate, I3 1,182

Cotton-root bark, 172

Couch grass, I7o

Counter-irritant, 18,38

Counter-irritation, 200

Cramp bark, 177

Cream of tartar, 143

Credé, unguentum, 180

Creolin, 170, I 96

Creosol, 87

Creosotal, 87

Creosote, 86 carbonate, 87 inhalant, 87 valerianate, I $8 \mathbf{I}$

Croton chloral, I 70 oil, 88

Cubebs, 170 
Cuca, 82

Culver's root, $\mathbf{1 7 3}$

Cupping, 205

dry, 205

wet, 206

Cusso, I 70

DEADLx nightshade, 62

Decoction, 12, 215

Definitions, I I

Delphinium, 176

Deodorized alcohol, 46

Depilatory, I 8

Dermatol, 65

Diachylon plaster, I I7

Diaphoretic, 18,37

Digestant, I8, 30

Digitalein, 89

Digitalin, 89

Digitalis, 89

Digitonin, 89

Digitoxin, 89

Dionin, 131

Diphtheria antitoxin, 212

Disinfectant, I8, 194

Disinfection, I97

of bedclothing, 199

of excreta, I99

of feces, 200

of urine, 200

Diuretic, I8, 34

stimulating, 34

systemic, 34

uses of, 35

Diuretin, I 80

Dog button, 129

Dolomol, I8o

Donovan's solution, 61, I23

Dormiol, I80

Dosage rules, 25 of drugs, 25

Dover's powder, I IO, I3I

Draught, black, I 76

Drug actions, I4 administration, $2 \mathbf{I}$

classification, 26

constituents, I I

preparations, 12

Drugs acting on alimentary system,
Drugs acting on circulatory system, 28

on excretory apparatus, 37

externally, 38

on eye, 35

on genital tract, 36

on heat mechanisrn, 37

on metabolism, 36

on nervous system, 32

on respiratory system, 26

on urinary tract, 34

Duboisin, 170

Dulcamara, 170

Duotal, Ioo

Ecbolic, 19

Echinacea, I 70

Eisenzucker, II 3

Elaterin, $\mathbf{I} 7 \mathbf{I}$

Elaterium, I 7 I

Elixir, I 2

Emetic, 19, 30

Emetin, I 10

Emmenagogue, 19, 36

Emplastrum, I3

Emulsion, I 2, 21 5

asafetida, I66, 2 10

chloroform, 78

cod-liver oil, 83

Endermic medication, 24

Enema, asafetida, 2 I0

glycerin, 2 10

nutritive, 2 Io

oil, 2 I I

ox-gall, I 34, 2 I 1

purgative, 2 I I

quassia, 2 I I

simple, 2 ro

soapsuds, 2 ro

starch-and-laudanum, 2 I I

turpentine, 210

Enemata, 210

Enteroclysis, I90

Eosote, I 8I

Epinephrin, 158

Epispastic, 19

Epsom salt, 120

Ergot, 91

Ergotin, 91

Erigeron oil, I 7 I 
Eriodictyon, 171

Erythroxylon, 82

Eserin, 138

Ether, 92

Ethyl alcohol, 45

bromid, I 7 I

chlorid, 171

oxid, 92

Eucain, 171

Eucalyptol, I7 I

Eucalyptus, I $7 \mathbf{I}$

Euonymus, 171

Eupatorium, I7I

Euquinin, I81

Exalgin, 181

Excreta disinfection, 199

Expectorant, 19, 27

Extract, 12, 215

aconite, 44

aloes, 48

apocynum, 166

arnica, 166

aspidium, 166

belladonna, 62

cannabis indica, 72

cascara, 73

cimicifuga, 169

cinchona, 80

colchicum, 84

colocynth, 169

conium, 169

cotton-root bark, 172

digitalis, 89

ergot, 9 I

euonymus, 171

frangula, $17 \mathbf{I}$

gentian, 97

Goulard's, I 16

hematoxylon, 172

hyoscyamus, 107

iris, 172

jalap, II 5

leptandra, 173

licorice, 99

nux vomica, 129

opium, 130

physostigma, 138

podophyllum, I4I

rhubarb, 148

suprarenal, 158

thyroid, 159
FeCES, disinfection, 200

Fel bovis, I 33

tauri, 133

Fennel, I 7 I

Ferrum. See Iron. reductum, I I I

Filix mas, I66

Flag, blue, 172 sweet, 167

Flaxseed poultice, 204

Fleabane, oil of, I 7 I

Fluidextract, 12, 215

absinth, I 65

aconite, 44

adonis vernalis, 165

allspice, 165

apocynum, 166

arnica, $\mathbf{1} 66$

aspidosperma, 166

belladonna, 62

boneset, 167

buchu, 167

cactus, 167

calamus, 167

calumba, 167

cannabis indica, 72

capsicum, 73

cardamom, 168

cascara, 75

castor oil, 76

caulophyllum, I66

celery, 168

chamomile, 167

cimicifuga, 169

cinchona, So

cinnamon, $\mathbf{1} 68$

cloves, 169

coca, 82

colchicum root, 84 seed, 84

conium, 169

convallaria, 86

corn-silk, I69

cotton-root bark, 172

cubebs, 170

cusso, I 70

digitalis, 89

echinacea, 170

ergot, 91

eriodictyon, I $7 \mathrm{I}$ 
Fluidextract, eucalyptus, I 7 I

fennel, I $7 \mathbf{I}$

frangula, 17 I

gelsemium, 96

gentian, 97

ginger, 97

grindelia, 172

hamamelis, 172

helonias, 172

humulus, 172

hydrastis, 102

hyoscyamus, 107

ipecac, I IO

iris, 172

juniper, 173

kamala, 173

kava kava, I73

kola, 173

kxameria, 173

lactucarium, 173

leptandra, 173

licorice, 99

lobelia, I73

Iupulin, 172

nux vomica, 129

passiflora, I 74

phytolacca, I 74

pilocarpus, 140

podophyllum, I4I

quassia, I 75

rhubarb, 148

sanguinaria, 175

santal wood, 175

saw palmetto, 175

scoparius, I 51

senega, 176

senna, 176

spigelia, 176

squill, I 55

stramonium, 176

sumbul, I 74

uva ursi, 176

valerian, 160

veratrum viride, $16 \mathrm{I}$

viburnum, 177

wild cherry, 174

witch hazel, 172

Fomentations, hot, 208

Formaldehyd, 93

fumigation, 198
Formalin, 93, I95

Formic aldehyd, 93

Formin, I81

Formol, 93

Fowler's solution, 59

Fox glove, 89

Frangula, 17 I

Friar's balsam, 64

Fumigation, 24 formaldehyd, 198

sulphur, 198

Galactagogue, $19,3^{8}$

Galla, 94

Gallic acid, 94

Gambir, 168

Gamboge, 172

Gastric lavage, I9I

Gaultheria, 95

Gelsemin, 96

Gelsemium, 96

Gentian, 97

Geosote, 100, I8I

Gin, 46

Ginger, 97

Glacial acetic acid, 42

Glauber salt, I 55

Glonoin, 128

Glucosid, definition of, I 2

Glusid, I 75

Glycerin, 98 enema, 210

Glycerite, 13, 216 boroglycerin, 66 carbolic acid, 73

hydrastis, 102

tannic acid, 94

Glycerophosphates, I 37

Glyceryl trinitrate, I 28

Glycyrrhiza, 99

Glycyrrhizin, 99

Goa powder, I68

Golden seal, IO2

Gossypii radicis cortex, 172

Goulard's cerate, I 16 extract, 116

Granatum, I42

Gray powder, 68, I 24

Green hellebore, I6I vitriol, I I 3 
Gregory's powder, 148

Griffith's mixture, I 2

Grindelia, I 72

Guaiac, I 72

Guaiacol, 87, 100 benzoate, 100 carbonate, I00 salicylate, I00 valerianate, I00, I8I

Guaranin, 67

Gum arabic, 165 camphor, 70

Hamamelis, I 72

Heart sedative, 28 stimulant, 28

Heat, application of, 206

Hedonal, i 8 I

Hellebore, American, I6r green, $16 \mathbf{I}$

Helmitol, 18r

Helonias, 172

Hematinic, I9

Hematoxylon, 172

Hemlock, I69

Hemostatic, 19

Hemostatin, 158

Hemp, Canadian, 166 Indian, 72

Henbane, 107

Heroin, I3I

Hexamethyleneamin, 183

Hirudo, IoI

Hive syrup, I 55

Hoffman's anodyne, 92

Holocain, 181

Homatropin, 102

Hops, 172

Hot baths, 208 fomentations, 208 packs, 208

Hot-air baths, 208

Hot-water bag, 207

Humulus, 172

Hydrargyrum. See Mercury.

Hydrastin, 103

Hydrastinin, IO3

Hydrastis, 102

Hydrated iron, I I 3

Hydrochloric acid, 103
Hydrochloric acid, dilute, 104 Hydrocyanic acid, 105 dilute, 105

Hydrogen dioxid, 106 peroxid, I06, 195

Hydrotherapy, 185

Hyoscin, 107

Hyoscyamin, 107

Hyoscyamus, 107

Hypnotic, 20, 33 administration of, 22

Hypodermic medication, 23, 192

Hypodermoclysis, I 88

Hypophosphites, I37

Hyrgol, I 8I

ICE-BAG, I 88

Ichthalbin, I $8 \mathbf{I}$

Ichthargin, I $8 \mathbf{I}$

Ichthoform, I 8I

Ichthyol, 107, 197

Indian hemp, 72

Infusion, I 3,2 I 6

cinchona, 80

digitalis, 89

quassia, 175

senna, compound, 176

wild cherry, I74

Ingluvin, 172

Inhalation, 24

Intestinal antiseptics, 3 I astringents, 32

Inunction, 24

Iodin, Io8

Iodism, Io9, I 46

Iodoform, Io9, I 96

Iodol, I $8 \mathbf{I}$

Ipecac, I ro

Iris, 172

Irisin, 172

Iron arsenate, II 2

bromid, I I 2

carbonate, II 2

mass, I I 2

saccharated, I I 2

chlorid, I 12

tincture, I I 2

citrate, II 2

compounds, III

glycerinophosphate, I37 
Iron, hydrated, 113

hypophosphite, 112, 137

iodid, I 13

syrup, II 3

oxid, 113

phosphate, I I $3, \mathbf{1}_{3} \mathrm{~S}$

Quevenne's, II I

reduced, III

sulphate, I 3

valerianate, $1_{3}, 16$ I

Itrol, 18 r

\section{JABORANDI, I $^{\circ}$}

Jalap, I 15

Jamaica dogwood, 173

Jasmine, yellow, 96

Jervin, $\mathbf{1 6 \mathbf { r }}$

Juniper, I73

KamaLA, $\mathbf{7 3}$

Kava kava, 173

Kino, 173

Kola, 173

Kousso, 170

Krameria, 173

LACTUCARIUM, 173

Lanolin, 173

Lanum, 173

Lard, benzoinated, 64

Laudanum, I 30

Laxative, 20, 31

Laxatives, administration of, 22

Lead acetate, I 6

carbonate, I 16

compounds, 116

iodid, I 7

oleate, II 7

oxid, II 7

plaster, I 7

sugar of, I 16

white, 116

Lead-and-alum lotion, 196

Leech, IOI

Leptandra, 173

Lettuce, 173

Licorice, 99

Lily of the valley, 86

Lime, 69 chlorinated, 70
Lime, sulphurated, 70

Lime-water, 69

Liniment, I3, 2 I 6

ammonia, 50

belladonna, 62

calcium, 70

camphor, 7 I

chloroform, 78

lime, 70

turpentine, 159

Liquor, 13 calcis, 69

Liquorice, 99

Litharge, II 7

Lithium benzoate, 64, I 8 bromid, 118

carbonate, 118

citrate, II 8

iodid, 119

salicylate, I19, 149

Lobelia, 173

Lobelin, I 73

Logwood, 172

Lotio flava, 122

nigra, 123

Lugol's solution, 108

Lunar caustic, 152

Lupulin, 172

Lycetol, I8I

Lysol, 182, 196

Magnesia, I 20

Magnesium carbonate, II9 compounds, I I9

glycerinophosphate, $\mathbf{I} 37$

oxid, I 20

sulphate, I 20

Male fern, 166

Mandrake, I 41

Manganese dioxid, r 20

hypophosphite, 137

oxid, black, I 20

peroxid, I 20

sulphate, 120

Mass, 216

blue, 124

copaiba, I 69

iron carbonate, 112

mercury, 124

Vallet's, II 2 


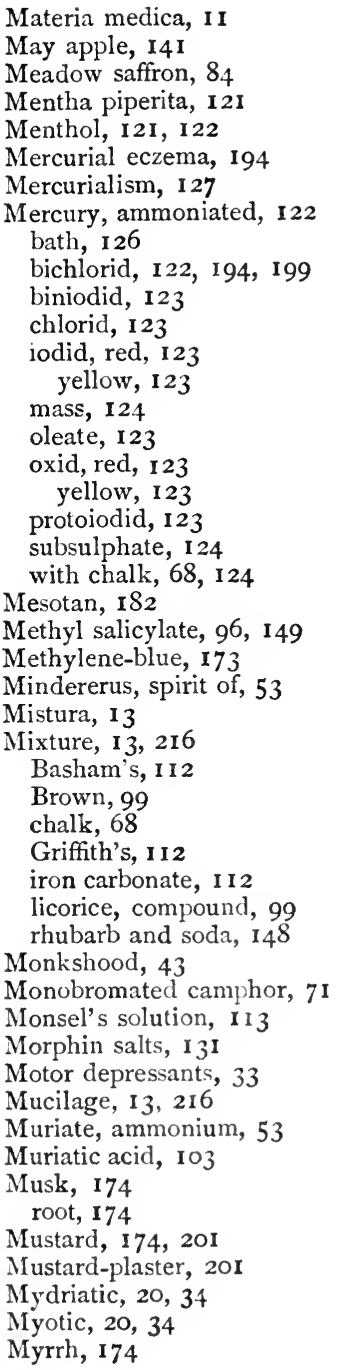

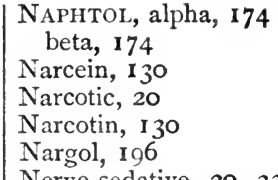

Nerve sedative, 20, 33

Night-blooming cereus, 167

Nightshade, deadly, 62

Niter, 146

Nitric acid, 127

Nitroglycerın, I 28

Nitrohydrochloric acid, 128

Normal saline solution, I 89

Nosophen, I 82

Nutgalls, 94

Nutritive enema, 2 Io

Nux vomica, 129

OrL, anise, 165 camphorated, $7 \mathbf{I}$

carron, 70

castor, 76

cinnamon, $\mathrm{I} 68$

clove, 169

cod-liver, 83

copaiba, I69

croton, 88

cubeb, I 70

erigeron, I $7 \mathbf{I}$

eucalyptus, I $7 \mathbf{I}$

fleabane, I $7 \mathrm{I}$

gaultheria, 95

juniper, 173

peppermint, I $2 \mathbf{I}$

phosphorus, 136

santal wood, 175

savin, 175

teaberry, 95

turpentine, 159, 197, 202

wintergreen, 95

Ointment, I 3, 2 I 6

belladonna, 62

blue, 124

carbolic acid, 73

collargol, iso

Credé, I 80

iodin, IoS

iodoform, I Io

lead carbonate, II 6 
Ointment, lead iodid, I I 7

mercury, 124

ammoniated, 122

oxid, red, 123

yellow, 123

nutgall, 94

red precipitate, 123

tannic acid, 94

white precipitate, 122

Oleate, I3, 2 I 7

lead, II 7

mercury, 123

Oleoresin, aspidium, I 66

capsicum, 73

cubeb, I 70

ginger, 98

Oleum morrhuæ, 83

ricini, 76

tiglii, 88

Opium, I30

preparations, I30

Orthoform, I82

Ox-gall, I33

enema, I34, 2 I I

Oxytocic, 20

PACKs, cold, 187 . hot, 208

Pancreatin, I 34

Papain, I34

Papayotin, I34

Paraldehyd, I 74

Paregoric, I3I

Parsley, I66

Passiflora, I 74

Passion flower, 174

Pearson's solution, 6I

Pelletierin tannate, $\mathbf{1 4 2}$

Pepo, 175

Pepper, Cayenne, 73 red, 73

Peppermint, I 2 I

Pepsin, 135

lactated, 135

powder, compound, 135

saccharated, 135

Perhydrol, 182

Peroxid, hydrogen, 106, 195

Peru, balsam of, 166

Peruvian bark, 80
Pharmacopeia, I I

Phenacetin, I 35

Phenic acid, 73

Phenol, 73

Phenyl alcohol, 73

Phenylacetamid, 40

Phosphates, $\mathbf{I}_{3} 8$

Phosphoric acid, I37

Phosphorus compounds, 136

Physostigma, I 38

Physostigmin, 138

Phytolacca, I74

Picratol, 182

Picric acid, I39

Picronitric acid, I39

Picrotoxin, 169

Pilocarpin, I 40

Pilocarpus, I 40

Pimenta, I65

Pink root, 176

Piperazin, I 82

Piscidia, 173

Plaster, 13, 21 7

belladonna, 62

cantharides, 202

capsicum, 73

diachylon, I 7

lead oxid, I 7

mercury, 124

with ammoniac, 124

mustard-, 201

opium, I $3 \mathbf{I}$

Spanish-fly, 202

spice, 202

Plumbism, I IS

Plumbum. See Lead.

Podophyllin, I4I

Podophyllum, I4I

Poison nut, 129

treatment of, 130

Poke berry, 174

root, 174

Pomegranate, 142

Potash alum, 49

Potassio-aluminum sulphate, 49

Potassium acetate, 142

and sodium tartrate, 146

arsenite, 142

solution, 59

bicarbonate, $\mathbf{I 4}^{2}$ 
Potassium bichromate, 142

bitartrate, 143

bromid, I43

chlorate, I 44

citrate, 145

glycerinophosphate, I37

hypophosphite, I37

iodid, 145

nitrate, 146

permanganate, 121 , 196

tartrate, acid, 143

Poultice, bread, 204

charcoal, 204

flaxseed, 204

spice, 202

Poultices, 204

Powder, 13

anise, 165

chalk, compound, 68

Dover's, I ro, I 3 I

effervescing, compound, 147 goa, 168

gray, 68, 124

Gregory's, I 48

ipecac and opium, IIo, I3I

jalap, compound, I I 5

licorice, compound, 99

opium, 130

pepsin, compound, I 35

purging, I 5

rhubarb, compound, $\mathbf{I}_{4} 8$

Seidlitz, 147

Proferrin, 182

Protan, 182

Protargol, 182, 196

Protoiodid of mercury, 123

Prunus virginiana, I74

Prussic acid, 105

Ptyalagogue, 20

Pulvis, 13

Pumpkin seed, 175

Purgative, 20, 3 I

cholagogue, $3 \mathbf{I}$

drastic, $3 \mathbf{I}$

enema, $2 \mathbf{I I}$

Purging powder, II 5

QUAKER button, I 29

Quassia, I 75

enema, 2 I I
Quevenne's iron, I I I

Quinin hypophosphite, $13^{8}$

salts, 8o

valerianate, $\mathbf{I} 6 \mathrm{I}$

REC'TAL alimentation, 209

medication, 209

Red mercury iodid, 123

oxid, I 23

precipitate, 123

wine, 46

Reduced iron, II I

Resin, copaiba, I69

jalap, I 15

podophyllum, i4r

scammony, I75

Resorcin, I47, 197

Respiratory stimulants, 27

Rhamnus purshiana, 75

Rhatany, I73

Rheum, 147

Rhubarb, 147

Rochelle salt, 146

Root, blood, I 75

Culver's, 173

pink, 176

poke, 174

Rubefacient, 20, $3^{8}$

Rum, 46

Russian bath, 209

Rye, spurred, 9I

SACCHARATED pepsin, I35

Saccharin, I 75

Saffron, 175 meadow, 84

Sal ammoniac, 53

Saleratus, 153

Salicin, I 49

Salicylates, I49

Salicylic acid, I49

Salicylism, I 5 I

Saline cathartics, $3 \mathbf{I}$ administration of, 23

Salipyrin, 182

Salol, 149

Salophen, 149

Salt, Epsom, I 20

Glauber, I55

Rochelle, 146 
Salt, volatile, 52

Saltpeter, 146

Sandal wood, 175

Sanguinaria, 175

Santal wood, I75

Santonica, 175

Santonin, 175

Saturnism, II 8

Savin, 175

Saw palmetto, I75

Scammony, I 75

Scilla, I 55

Scoparius, I 5 I

Sedative, cardiac, 17,28 nerve, 20,33

Seidlitz powder, 147

Senega, 176

Senna, 176

Sialagogue, 21, 37

Silver citrate, I8I

gelatose, I 79

lactate, 178

nitrate, 152, 196

Sinapis, 174

Sitz-bath, 208

Snakeroot, black, 168

Soapsuds enema, 2 10

Soda, caustic, 154

Sodium arsenate, 61, 153

benzoate, 64,153

biborate, 66

bicarbonate, 153

borate, 66,154

bromid, 154

cacodylate, I 54

compounds, I 53

glycerinophosphate, 137

hydrate, 154

hydroxid, 154

hypophosphite, I 38

hyposulphite, 155

iodid, 154

nitrite, 154

- phosphate, 138, I54

salicylate, I49, I 54

sulphate, 155

sulphite, 155

thiosulphate, 155

valerianate, $16 \mathbf{1}$

Solution, I 3, 2 I 7
Solution, ammonium acetate, 53

arsenic and mercury iodid, $6 \mathbf{I}$, 123

arsenous acid, 59

Donovan's, 61, 123

Fowler's, 59

iodin, compound, 108

iron and ammonium acetate, I I 2

lead subacetate, I 16

Lugol's, 108

Monsel's, II3

normal saline, I 89

Pearson's, 6r

potassium arsenite, 59

saline, normal, I89

sodium arsenate, 6I

trinitrin, 128

Somnifacient, 21

Soporific, 21

Spanish fly, 167, 202

Spartein, I5I

Spice-plaster or poultice, 202

Spigelia, 176

Spirit, I4, 217

ammonia, 5 I aromatic, $5 \mathrm{I}$

camphor, 70

chloroform, 78

ether, 92

compound, 92

gaultheria, 95

glonoin, 128

juniper, 173

compound, 46

mindererus, 53

nitroglycerin, 128

of wine, 45

peppermint, 122

phosphorus, I $_{3} 6$

wintergreen, 95

Spiritus frumenti, 46

juniperis comp., 46

vini gallici, 46

Sponge-bath, I 85

Sputum, disinfection, 199

Squill, 155

Staphisagria, 176

Starch-and-laudanum enema, 2 II

Stavesacre, 176

Stearopten, 12 
Stimulant, cardiac, 17,28 respiratory, 27 urinary, 35

Stomachic, 21, 29

Stramonium, 176

Strontium compounds, 156 lactate, 156

Strophanthin, 156

Strophanthus, 156

Strychnin, 129

salts, 129

Stupe, turpentine, 205 and chloroform, 205

Stupes, 205

Styptic, 2 I collodion, 94

Stypticin, 182

Sublamin, 183

Succinic peroxid, I79

Sudorific, 21

Sugar of lead, 116

Sulfonal, 176

Sulphur, 176 fumigation, 198

Sulphuric acid, 157 aromatic, 157

Sumbul, 174

Suppository, I4

Suprarenal substance, 157

Suprarenalin, 158

Sweet flag, 167

Synonyms, 220

Syrup, 14, 21 8

eriodictyon aromatic, I $7 \mathbf{I}$

ginger, 98

hive, 155

ipecac, I 10

iron iodid, 113

krameria, 173

lime, 70

rhubarb, 148

aromatic, 148

senega, 176

squill, I 55

compound, 55, I 55

tolu, $x 76$

wild cherry, $\mathbf{1} 74$

TABLE of drug strengths, 215 of synonyms, 220

Table of weights and measures, 224

Tannalbin, 183

Tannic acid, 94

Tannigen, 183

Tannin, 94

Tannocol, 183

Tannoform, 183

Tartar emetic, 55

Tartrated antimony, 55

Teaberry, oil of, 95

Teniacide, 2 I

Terpin hydrate, 176

Tetanus antitoxin, 213

Thein, 67

Theocin, 183

Therapeutics, I I

Thiocol, 183

Thiosinamin, 183

Thymol, 176

Thyroid gland, I 59

Tincture, 14,218

aconite, 44

aloes, 48

and myrrh, 48

arnica, 166

asafetida, 166

benzoin, 64 compound, 64

calumba, 167

cannabis indica, 72

cantharides, 167

capsicum, 73

cardamom, compound, I68

catechu, compound, I68

cinchona, 80

colchicum, $\mathrm{S}_{4}$

cubeb, 170

digitalis, 89

gelsemium, 96

gentian, compound, 97

ginger, 97

guaiac, 172

ainmoniated, $\mathbf{1 7 2}$

humulus, $\mathbf{I} 72$

hydrastis, IO2

hyoscyamus, 107

iodin, 108

ipecac-and-opium, x 10

iron clilorid, 112

kino, 173 
Tincture, krameria, 173

lobelia, I73

musk, I 74

myrrh, 174

nutgall, 94

nux vomica, 129

opium, I 30

camphorated, I3I

deodorized, I 30

physostigma, 138

quassia, 175

rhubarb, 148

aromatic, 148

sweet, 148

sanguinaria, I 75

squill, I 55

stramonium, 176

strophanthus, I 56

valerian, 160

ammoniated, I6I

veratrum viride, $\mathbf{r} 6 \mathbf{I}$

Tolu, 176

Toxicology, I I

Tricresol, 183

Trinitrin, 128

Trinitrophenol, I 39

Trional, 176

Triticum, I 70

Tub-bath, I 86

'Turkish bath, 208

Turpentine, enema, 2 ro oil of, 1 59, 197, 202 stupes, 205

Turpeth mineral, 124

UNGUENTUM, 13 Credé, 180

Unicorn root, false, I 72

Urinary antiseptics, 35 stimulants, 35

Urine disinfection, 200

Urotropin, 183

Uva ursi, 176

VALERIAN, I6o

Valerianates, I6I

Vallet's mass, 112

Vasoconstrictor, 21, 29

Vasodilator, 21, 29

Veratrum viride, I6I
Veronal, 183

Vesicant, 21, 38

Viburnum opulus, 177

prunifolium, 177

Vinegar, I4, 42, 218

opium, I3 I

squill, I 55

Vinum, 14

album, 46

rubrum, 46

Vitriol, blue, 169

elixir of, 157

green, I 3

oil of, 157

white, 163

Volatile salt, 52

Vulneraries, 39

WAHOO, I 7 I

Wash, black, I 23

yellow, 122

Water, I4, 218

ammonia-, 50

stronger, 5 I

camphor-, 70

chloroform-, 78

creosote-, 87

lime-, 69

oxygenized, 106

peppermint-, 122

Weights and measures, 224

Whisky, 46

White lead, I 6

precipitate, 122

vitriol, 163

Wild cherry, I 74

Wine, 14, 219

antimony, 55

colchicum root, 84

seed, 84

ergot, $9 \mathbf{I}$

ipecac, I Io

opium, I $3 \mathbf{I}$

red, 46

spirit of, 45

white, 46

Wines, 46

Wintergreen, oil of, 95 artificial, 96, 149

Witch hazel, 172 
Wolfsbane, 43

Wormseed, levant, I 75

Wormwood, 165

XEROFORM, 184

YeLLOW jasmine, 96 root, 102 wash, 122

Yerba santa, I 71

Yohimbin, I84

ZEA mays, 169

Zinc bromid, 163
Zinc butter, 162

carbonate, 162

chlorid, 162

compounds, $\mathbf{I} 62$

iodid, 163

oxid, 163

permanganate, I 64

phenol sulphonate, 164

phosphid, 138,164

sulphate, I 63

sulphocarbolate, 164 valerianate, I6I, I64 


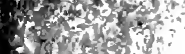

26

(2)

a. 12

a.

ats

s.

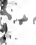




$$
\cdot
$$




\section{SAUNDERS’ BOOKS}

\section{for

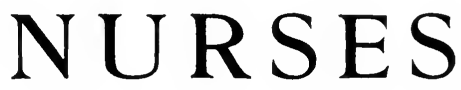

Books Sent, Carriage Paid, upon Receipt of Price

PAGE

Abbott's Hygiene of Transmissible Diseases . . . . . . 8

Barton and Wells' Medical Thesaurus . . . . . . . . 8

Beck's Reference Handbook for Nurses. . . . . . . . . . 4

Bergey's Hygiene. . . . . . . . . . . . . . . 8

Davis' Obstetric and Gynecologic Nursing . . . . . . . 5

DeLee's Obstetrics for Nurses . . . . . . . . . . . . . . 5

Dorland's American Illustrated Dictionary. . . . . . . . 8

Dorland's American Pocket Medical Dictionary . . . . 6

Fowler's Operating Room and Patient . . . . . . . . . . 4

Friedenwald and Ruhrah on Diet. . . . . . . . . . 6

Grafstrom's Mechanotherapy (Massage) . . . . . . . . 6

Griffith's Care of the Baby. . . . . . . . . . . . . 7

Lewis' Anatomy and Physiology for Nurses. . . . . . . 3

Martin's Minor Surgery, Venereal Diseases, and Bandaging 4

Morris' Essentials of Materia Medica . . . . . . . . . 7

Morrow's Immediate Care of the Injured . . . . . . . . . 3

Nancrede's Essentials of Anatomy . . . . . . . . . . . . 3

Paul's Nursing in the Acute Infectious Ferers . . . . . 5

Pyle's Personal Hygiene. . . . . . . . . . . . . . . . 8

Stevens' Practice of Medicine. . . . . . . . . . . . . . 7

Stoney's Bacteriology and Surgical Technic. . . . . . . 2

Stoney's Materia Medica for Nurses . . . . . . . . . . . 2

Stoney's Nursing . . . . . . . . . . . . . . . . . . . 2

Williams' Essentials of Practice. . . . . . . . . . . 7

\section{W. B. SA UNDER S C OMPANY \\ 925 Walnut Street




\section{Stoney's Nursing}

In this excellent volume the author explains the entire range of private nursing as distinguished from hospital nursing; and the nurse is given definite directions how best to meet the various emergencies. The American Journal of Nursing says it "is the fullest and most complete" and "may well be recommended as being of great general usefulness. The best chapter is the one on observation of symptoms which is very thorough." There are directions how to improvise everything ordinarily needed in the sick room.

Practical Points in Nursing. By Emily M. A. Stoney, Superintendent of the Training School for Nurses in the Carney Hospital, South Boston, Mass. 12mo, 466 pages, illustrated. Cloth, $\$$ r.75 net.

\section{Stoney's Materia Medica NEw (3d) EDTIIION}

Stoney's Materia Medica was written by a head nurse who knows just what the nurse needs. American Medicine says it contains "all the information in regards to drugs that a nurse should possess. $* * *$ The treatment of poisoning is stated in a manner that will permit of its being carried out thoroughly and intelligently."

Materia Medica for Nurses. By Emily M. A. STONeY, Superintendent of the Training School for Nurses in the Carney Hospital, South Boston, Mass. $12 \mathrm{mo}$ volume of 300 pages. Cloth, \$r.50 net.

\section{Stoney's Surgical Technic}

The first part of the book is devoted to Bacteriology and Antiseptics; the second part to Surgical Technic, Signs of Death, Autopsies, Bandaging and Dressings, Obstetric Nursing, Care of Infants, etc., Hygiene and Personal Conduct of the Nurse, etc. The New York Medical Record says it " is a very practical book which presents the subjects stated in its title in a concise manner."

Bacteriology and Surgical Technic for Nurses. By EMILY M. A. Stoney. Revised by Frederic R. GRIffith, M. D., New York. $12 \mathrm{mo}$ volume of 278 pages, fully illustrated. Cloth, \$1.50 net. 


\section{Lewis' Anatomy and Physiology}

JUST ISSUED

This book is the outcome of a wide demand for such a workone that would treat anatomy and physiology from the murse's point of view. Dr. Lewis has based the plan and scope of his work on the methods employed by him in teaching these subjects to nurses. The Nurses Journal of the Pacific Coast says "it is not in any sense rudimentary, but comprehensive in its treatment of the subjects in hand." The application of the knowledge of anatomy and physiology in the care of the patient is emphasized. The text is made more clear by the practical illustrations, a number in colors.

Anatomy and Physiology for Nurses. By LeRoy Lewis, M. D., Lecturer on Anatomy and Physiology for Nurses, Lewis Hospital, Bay City, Mich. 12 mo of $3^{17}$ pages, 146 illustrations. Cloth, $\$ 1.75$ net.

\section{Morrow's Immediate Care of Injured} JUST ISSUED

There has long been a demand for a modern work on first aid that would tell the nurse just what to do in any emergency. Dr. Morrow's book is just such a work. There are chapters on bandaging, practical remedies, accidents and emergencies, hemorrhages, burns and scalds, sprains, poisons and their antidotes, etc.

Immediate Care of the Injured. By ALBERT S. MORROW, M. D., Attending Surgeon to the New York City Home for the Aged and Infirm. Octavo of 340 pages, with 238 illustrations. Cloth, \$2.50 net.

\section{Nancrede's Anatomy}

NEW ( 7 th) EDITION

In this revision the entire book has been carefully gone orer and the section on the Nervous System completely rewritten. The American Joumal of the Medical Sciences says "it is one of the best of all the question compends and will no doubt continue to enjoy its deserved success."

Essentials of Anatomy. Charles B. G. DeNanCrede, M. D., Professor of Surgery and Clinical Surgery in the University of Michigan, Ann Arbor. I2mo, 400 pages, 180 illustrations. Cloth, $\$$ r.oo net. 


\section{Fowler's Operating Room}

Dr. Fowler's work contains all information of a surgical nature that a nurse must know in order to attain the highest efficiency. Canadian Journal of Medicine and Surgery says: "We find compactly and clearly stated just those thousand and one things which are scattered in journals, and which when required are so hard to locate. No proceeding is advised which has not been amply tested."

The Operating Room and the Patient. By Russell S. FOWLeR, M. D., Surgeon to the German Hospital, Brooklyn, New York. Octavo volume of 172 pages, with original illustrations. Cloth, $\$ 2.00$ net.

\section{Martin's Minor Surgery}

NEW (2d) EDITION

Nurses will find this book of much value. Full directions for applying the many kinds of bandages are given and clearly illustrated; and all the technic of minor surgery that a nurse must know is plainly presented. The New York Medical Record says: "This is one of the most practical books that one can desire. The illustrations are neat and clear."

Essentials of Minor Surgery, Bandaging and Venereal Diseases. By EDWARD MARTIN, M. D., Professor of Clinical Surgery, University of Pennsylvanla. I2mo, r66 pages, illustrated. Cloth, $\$ 1, \infty$ net.

\section{Beck's Reference Handbook}

This little book contains all the information that a nurse requires to carry out any directions given by the physician. The Montreal Medical Journal says it is " cleverly systematized and shows close observation of the sickroom and hospital regime. The book can be recommended."

A Reference Handbook for Nurses. By Amanda K. BeCK, Graduate of the Illinois Training School for Nurses, Chicago, Ill. $32 \mathrm{mo}$ volume of 177 pages. Bound in flexible morocco, $\$ \mathbf{1 . 2 5}$ net. 


\title{
DeLee's Obstetrics for Nurses
}

\author{
JUST ISSUED-NEW (2d) EDITION
}

Dr. DeLee treats obstetrics from the nurse's point of view. The book really considers two subjects-obstetrics for nurses and the actual obstetric nursing. Trained Nurse and Hospital Review says the "book abounds with practical suggestions, and they are given with such clearness that they cannot fail to leave their impress upon the mind of the reader." The practical illustrations are nearly all original, having been made specially for this work.

Obstetrics for Nurses. By Joseph B. DeLee, M. D., Professor of Obstetrics at the Northwestern University Medical School, Chicago. I2mo volume of 460 pages, fully illustrated. Cloth, $\$ 2.50$ net.

\section{Davis' Obstetric \& Gynecologic Nursing}

\section{RECENTLY ISSUED-NEW (2d) EDITION}

Obstetric nursing demands some knowledge of natural pregnancy and of the signs of accidents and diseases which may occur during pregnancy and labor. The Trained Nurse and Hospital Review says: "This is one of the most practical and useful books ever presented to the nursing profession." Gynecologic nursing is really a branch of surgical nursing, and as such requires special instruction and training.

Obstetric and Gynecologic Nursing. By EDWARD P. Davis, M. D., Professor of Obstetrics in the Jefferson Medical College, Philadelphia. I2mo volume of 402 pages, illustrated. Buckram, $\$$ I.75 net.

\section{Paul's Fever Nursing}

JUST ISSUED

Dr. Paul has laid great stress upon the care and management of each disease, as this relates directly to the duties of the nurse. The London Lancet thinks " the book is an excellent one and will be of value to those for whom it is intended. The text is clear and full, and the illustrations are good." 


\section{Friedenwald and Ruhrah's Dietetics for Nurses \\ JUST ISSUED}

This work has been prepared to meet the needs of the nurse, both in the training school and after graduation. It aims to give the essentials of dietetics, considering briefly the physiology of digestion and the various classes of foods. American Joumal of Nursing says it " is exactly the book for which nurses and others have long and vainly sought. A simple manual of dietetics, which does not turn into a cook-book at the end of the first or second chapter."

Dietetics for Nurses. By Julius Friedenwald, M. D., Clinical Professor of Diseases of the Stomach, and JOHN RUhRAH, M. D., Clinical Professor of Diseases of Children, College of Physicians and Surgeons, Baltimore. r2mo volume of 365 pages. Cloth, $\$$ r.50 net.

\section{American Pocket Dictionary}

This is the ideal pocket lexicon. It contains a complete vocabulary, defining all the terms of modern medicine. The Trained Nurse and Hospital Review says: "We have had many occasions to refer to this dictionary, and in every instance we have found the desired information." The work also contains a wealth of anatomic tables of value to nurses.

Dorland's Pocket Medical Dictionary. Edited by W. A. NewmaN DORLAND, M. D., of the University of Pennsylvania. rlexible leather, with gold edges, $\$$ r. $\infty 0$ net; with patent thumb index, $\$$ r. 25 net.

\section{Grafstrom's Mechano-therapy NEw} The Boston. Hedical and Surgical Journal says : "It states in concise language the various methods which by long experience have been found useful in treament by mechanical means."

Mechano-Therapy (Massage and Medical Gymnastics). By Axel V. Grafstrom, B. Sc., M. D., Attending Physician, Gustavus Adolphus Orphanage, Jamestown, N. Y. I2mo, 200 pages. Cloth, \$1.25 net.

\section{Friedenwald \& Ruhrah on Diet $\begin{gathered}\text { JUST ISSUED } \\ (2 d) \text { EDIrION }\end{gathered}$}

Diet in Health and Disease. By JUI,IUS FRIEDENWAI,D, M. D., Clinical Professor of Diseases of the Stomach, and JoHx RuHRAH, M. I)., Clinical Professor of Diseases of Children, College of Physicians and Surgeons, Baltimore. Octavo volume of 725 pages. Cloth, $\$ 4.00$ net. 


\section{Stevens' Practice}

During his absence the physician depends entirely upon the knowledge and watchfulness of the nurse to report to him any changes that may have occurred in the patient's condition. Of this work the Buffalo Medical Journal says: "Its arrangement is excellent, being such as to facilitate ready reference to its multifarious subjects.' Dr. Stevens has brought within a comparatively small compass a complete outline of the practice of medicine.

Practice of Medicine. By A. A. Stevens, M. D., Professor of Materia Medica, Therapeutics, and Clinical Medicine, Wornan's Medical College. r2mo volume of 556 pages. Flexible leather, \$2.50 net.

\section{Williams' Practice}

JUST ISSUED

Throughout this book special stress has been laid on differential diagnosis, symptomatology, and treatment. The New York Medical New's says " the symptoms are particularly well abstracted, and give the book real value."

Essentials of the Practice of Medicine. By WilliaM R. WILlians M. D., formerly Instructor in Medicine and Lecturer in Hygiene, Cornell University. r2mo, 46r pages. Double number. Cloth, \$r.75 net.

\section{Morris' Materia Medica}

The Trained Nurse and Hospital Review says: "The work is thoroughly up to date, well arranged, compact, and yet contains a very large amount of matter.'”

Essentials of Materia Medica, Therapeutics, and Prescription Writing. By HeNRY MORRIS, M. D. Reviszd by W. A. BASTedo, M. D. Instructor in Materia Medica and Pharmacology at the Columbia University, New York. $12 \mathrm{mo}$ of 300 pages. Cloth; $\$$ r.00 net.

\section{Griffith's Care of the Baby}

NEW $3 \mathrm{~d}$ EDITION

The New York Medical Joumal says: "We are confident if this little work could find its way into the hands of every trained nurse, infant mortality would be lessened by at least fifty per cent.

The Care of the Baby. By J. P. CROzer GRIffith, M. D., Clinical Professor of Diseases of Chiluren, University of Pennsylvania. ramo of 436 pages, illustrated, including 5 plates. Cloth, \$r.50 net 


\section{Dorland's Illustrated Dictionary}

\section{JUST ISSUED-NEW ( 4 th) EDITION-2000 NEW TERMS}

This edition contains over 200 new terms. Dr. Howard $A$. Kelly says: "Dr. Dorland's Dictionary is admirable. It is so well gotten up and of such convenient size. No errors have been found in my use of it."'

The American Illustrated Medical Dictionary A Dictionary of the terms used in Medicine, Surgery, Dentistry, Pharmacy, Chemistry. and kindred branches: with Ioo new and elaborate tables. By W. A. N. DORLAND, M. D. Large octavo of 836 pages, 293 illustrations, Ir in colors. Flexible leather, $\$ 4.50$ net: thumb index, $\$ 5.00$ net.

\section{Bergey's Hygiene}

NEW (2d) EDITION

The American Joumal of the Medical Sciences says this work "presents in compact form a very clear exposition of the general principles of hygiene," and "especially to be commended is the chapter on vital causes of disease."

The Principles of Hygiene. By D. H. Bergey, A. M., M. D., Assistant Professor of Bacteriology in the University of Pennsylvania. Octavo volume of 536 pages, fully illustrated. Cloth, $\$ 3.00$ net.

\section{Abbott's Transmissible Diseases (2d) EDITION}

In speaking of Dr. Abbott's book the Johns Hopkins Hospital Bulletin says: "The book is well calculated to meet a want which has long been felt by physicians and nurses."

The Hygiene of Transmissible Diseases. Their Causation, Modes of Dissemination, and Methods of Prevention. By A. C. ABвOT, M.D., Professor of Hygiene and Bacteriology, University of Pennsylvania. Octavo volume of 3 II pages, fully illustrated. Cloth, $\$ 2.50$ net.

\section{Pyle's Personal Hygiene}

A Manual of Personal Hygiene. Proper Living upon a Physiologic Basis. Edited by WALTER L. PYLE, A. M., M. D., Assistant Surgeon to Wills' Eye Hospital, Philadelphia. Octavo, 350 pages. Illustrated. $\$ 1.50$ net.

\section{Barton and Wells' Thesaurus unigue work}

A Thesaurus of Medical Words and Phrases. By WILFRED MI. BARTON, M. D., and WALTHR A. WELLS, II. D., of Georgetown University, Washington, D. C. 12 mo of 534 pages. Flexible leather $\$ 2.50$ net; with thumb index, $\$ 3.00$ net. 


PLEASE DO NOT REMOVE

CARDS OR SLIPS FROM THIS POCKET

\section{UNIVERSITY OF TORONTO LIBRARY}


